\title{
7th European Workshop on Astrobiology
}

\author{
EANA - European Astrobiology Network Association \\ October 22-24, 2007 \\ Turku, Finland
}

\section{PROGRAM}

\section{Sunday October 21}

Registration 18:00

Public lecture: First and Second Origin of Life 19:00

André Brack (Orleans, France), Tetsuya Yomo (Osaka, Japan)

\section{Monday October 22}

Registration

08:00

Welcome

9.00-9.20

Session Chair: Harry Lehto

$9.20-10.20$

1. Astrobiology, Life and Habitable zones

1.1 Updating the Astrobiology Roadmap Carl Pilcher

1.2 Life, the Universe and habitable zones Rami Rekola

1.3 The quest for terrestrial planets Ewa Szuszkiewicz

Coffee

$10.20-10.50$

Session Chair: Werner von Bloh $10.50-12.10$

2. Life, climate and the atmospheres

2.1 Biological feedbacks as cause and demise of Neoproterozoic icehouse: implications for multicellular evolution *P. Janhunen, H. Kaartokallio, I. Oksanen, K. Lehto, H. Lehto

2.2 Life and climate: is there a link? Peter Ditlevsen

2.3 The importance of high $\mathrm{CO}_{2}$ amounts in young terrestrial planetary atmospheres

*H. Lammer, M. L. Khodachenko, M. Panchenko, N. Terada, Yu.N. Kulikov

2.4 The life span of a photosynthetic-active biosphere on super-Earth planets

${ }^{*}$ C. Bounama, W. von Bloh, S. Franck

Lunch and EANA executive council meeting

$12.10-14.00$

Session Chair: Maria Webb

$14.00-15.20$
3. Mars research

3.1 Detection of anticipated life on Mars by Phoenix

*Joop M. Houtkooper and Dirk Schulze-Makuch

3.2 Methane and formaldehyde: their abundance and sources on the Earth and Mars

${ }^{*}$ C. Muller and D. Moreau

3.3 Geological and paleo-climatic constraints for the search of life on Mars

*V.-P. Kostama, M. Aittola, J. Korteniemi, H. Lahtela,

T. Törmänen, T. Öhman, J. Raitala

3.4 Modelling the surface and subsurface Martian radiation environment: Implications for Astrobiology

*L. R. Dartnell, L. Desorgher, J. M. Ward, A. J. Coates

Coffee

$15.20-15.50$

Session Chair: Frances Westall

$15.50-17.10$

4. Early life

4.1 The origin and evolution of viruses

D. Bamford

4.2 Horizontal gene transfer: evolution by default or product of natural selection? Anthony Poole

4.3 Membranes and the origin of cellular life *Tiina Laiterä and Kirsi Lehto

4.4 Elaborate morphologies in probable microfossils from Archaean chert, Pilbara Craton, Western Australia: indicators of complex cell behaviour?

*K. Sugitani, K. Grey, T. Nagaoka, K. Mimura,

M. R. Walter

\subsection{0-21.00 Poster session and refreshments}

\section{Tuesday October 23}

Session Chair: Axel Brandenburg $9.00-10.20$

5. Prebiotic chemistry

5.1 Conformation-dependent racemization of aspartyl residues in model peptides

*A. Brack, K. Kuge, N. Fujii

5.2 Driving chirality

H. Lehto

5.3 Emergence of chirality and molecular evolution *Raphaël Plasson, Hugues Bersini, Axel Brandenburg

5.4 A terrestrial prebiotic route to pyrroles and higher aromatics ${ }^{*}$ H. Strasdeit, S. Fox, D. Denysenko 
Coffee

$10.20-10.50$

Session Chair: Jean-Francois Lambert $10.50-12.10$

5.5 Oxidative pathways of reduced phosphorus compounds: a primary source of condensed phosphates on the early Earth *M. A. Pasek, T. P. Kee, J. I. Lunine

5.6 Phosphorus redox chemistry in planetary environments: implications for the origins of life

D. E. Bryant, I. B. Gorrell, D. Greenfield, S. M. Evans, L. Wang, D. E. Heard, M. A. Blitz, A. Goddard, C. Smith, M. A. Pasek, *T. P. Kee

5.7 Abiotic organic synthesis underneath the ocean floor Nils G. Holm

6. Small bodies in the Solar System: Comets, Meteorites, Moons

6.1 Unbiased meteorite statistics: an impossible task? The Oman experience

*Beda A. Hofmann, Edwin Gnos, Manuel Eggimann,

Ali Al-Kathiri

Lunch and EANA executive council meeting

$12.10-14.00$

Session Chair: Yves Ellinger

$14.00-15.20$

6.2 Organics on Wild 2 Comet: laboratory results of stardust samples

*J. R. Brucato and the Stardust Team

6.3 Composition of Saturnian E-ring Particles. Probing subsurface Oceans of Enceladus?

*F. Postberg, S. Kempf, R. Srama, E. Grün, J. K. Hillier,

S. F. Green, N. McBride

6.4 Interplay of novel organic and inorganic chemistry on Titan Sam Abbas

6.5 Photochemistry of organic molecules related to Mars, Titan, Meteorites and Comets: experiments in Terrestrial orbit and laboratory simulations

*Y. Y. Guan, H. Cottin, P. Coll, D. Coscia, N. Fray,

F. Macari, F. Stalport, F. Raulin, C. Szopa, D. Chaput, M. Viso, M. Bertrand, A. Chabin, L. Thirkell, F. Westall, A. Brack

Coffee

$15.20-15.40$

\section{EANA General assembly}

$15.40-16.20$

\section{Workshop Dinner and Sauna 16.30/18.45-23.00}

For participants for the sauna, the departure will be approx at 16.30 directly from Educarium.

The dinner only people will join the group later. The bus leaves at 18.45 sharp at the Orthodox Church by the Market place and at 19.00 at Caribia hotel.

Return is scheduled for 23.00. Further details will be given at the meeting.

\section{Wednesday October 24}

Session Chair: Gerda Horneck

$9.00-10.20$
7. Life in extreme conditions

7.1 Microbial life in extreme subglacial Antarctic lake environments: Lake Vostok

*S. Bulat, I. Alekhina, V. Lipenkov, V. Lukin, D. Marie,

C. Lavire, P. Normand, J. R. Petit

7.2 The enigma of the nitrate deposits in the Atacama Desert, Chile

*Rocco L. Mancinelli, Kimberley Warren-Rhodes, Amos Banin, Ragnhild Landheim

7.3 UV-induced DNA damage in Deinococcus radiodurans: photoproducts and gene expression

*P. Rettberg, U. Pogoda de la Vega, T. Douki, J. Cadet,

G. Reitz

7.4 Growth of microorganisms at Martian subsurface conditions: laboratory modelling

*A. K. Pavlov, V. N. Shelegedin, M. A. Vdovina,

A. V. Tretyakov

Coffee

$10.20-10.50$

Session Chair: Kirsi Lehto

$10.50-12.10$

7.5 New insights into the microbial diversity in spacecraft assembly clean rooms and the impact on planetary protection ${ }^{*}$ C. Moissl, Kasturi Venkateswaran, Gerhard Kminek

\section{Lithopanspermia}

8.1 Shock experiments in support of the Lithopanspermia theory: The influence of host rock composition, temperature and shock pressure on the survival rate of endolithic and epilithic microorganisms

${ }^{*}$ C. Meyer, D. Stöffler, M. Misgaiski, J. Fritz, R. Moeller,

E. Rabbow, G. Horneck, J.-P. De Vera, C. Cockell,

U. Hornemann

9. Education and Public Outreach

9.1 The science and culture of astrobiology in education and outreach

Mark Brake and Martin Griffiths

9.2 ABC-Net, a European astrobiology lecture course network *Gerda Horneck and the ABC-Net Team

Formal Ending of the meeting

(Lunch)

$12.10-13.35$

Y Young astrobiologist sessions

Youth Session Chair: Liisa Gunnelius $13.35-15.05$

Y.1 The origin of introns and mRNA

*Marc P. Hoeppner, Daniel C. Jeffares, Anthony M. Poole

Y.2 The importance of the Astrobiology for the young generations José Ruiz de la Herrán

Y.3 Irradiation of a homogeneous mixture of ammonia and carbon dioxide $\left(\mathrm{NH}_{3}, \mathrm{CO}_{2}\right)$ at low temperatures

*Sohan Jheeta, Anne LaFosse, Bhalamurugan Sivaraman, Slywia Ptasinska, Nigel Mason

Y.4 Dissociative recombination of nitrile ions - important processes in Titan's atmosphere 50

*Erik Vigren, Magdalena Kaminska, Vitali Zhaunerchyk, Mathias Hamberg, Mathias Danielsson, Richard D. Thomas, Jacek Semaniak, Patrik Andersson, Mats Larsson, Wolf D. Geppert 
Coffee

$15.05-15.30$

Youth Session Chair: Marc Hoeppner

$15.30-17.10$

Y.5 Compound model to explain water origins for Earth-like planets *Karla de Souza Torres and Othon Cabo Winter

Y.6 Structure and evolution of RNA polymerases Liisa Gunnelius
Y.7 Analysis of the critical moments of the evolution of biosphere macroparameters for revealing catastrophes *V. A. Ozheredov and N. G. Khorseva

Y.8 Searching for habitable-zone planets with SuperWASP *D. R. Anderson and The SuperWASP Consortium

Y.9 How to close the door leaving it open? - On the origin of membrane transport system Katarzyna Adamala

\section{TALKS}

\section{Astrobiology, Life and Habitable zones}

\author{
I.I Updating the Astrobiology Roadmap \\ Carl Pilcher \\ NASA Astrobiology Institute, USA
}

\section{I.2 Life, the Universe and habitable zones Rami Rekola \\ Tuorla Observatory, University of Turku, Finland}

We have only one example of a biosphere we know in the entire Universe. This is slightly surprising as we also know the Universe is enormous in size, possibly even infinite. There are more than a hundred billion stars in our own Galaxy. Even our Solar System has several planets, many moons, and a multitude of smaller objects. Why have we not seen or heard of life elsewhere? On the other hand, the Universe indeed is enormous. Distances between stars are currently beyond any capabilities we have of travelling in space. Even planetary research in our Solar System is really taking its first steps. We may have close neighbours, at least in the form of microbial life, which we know nothing about yet. A more educated viewpoint directs us to the source of our loneliness. Life cannot exist just anywhere. Some environmental criteria must be fulfilled for life to be born, to survive, to evolve and to thrive. Places suitable for life are called habitable zones. There is a multitude of ways habitable zones may be defined and clearly this is a crucial question in the search for extraterrestrial life. We can increase our chances in finding life - or at least decrease the time it takes - by restricting our search to locations where life is possible in the first place. Naturally we still have to answer the most fundamental question before we are able to define habitable zones. What is life anyway?

\section{I.3 The quest for terrestrial planets}

E. Szuszkiewicz

Institute of Physics and CASA*, University of Szczecin, Wielkopolska 15, 70-451 Szczecin, Poland

It is the best time now to search for terrestrial planets. The microlensing and high-precision radial velocity methods used from the ground may bring new discoveries at any time. Also a network of small groundbased telescopes is able to detect Earth-like planets using Transit Timing Variation techniques. The space mission COROT (COnvection ROtation and Transits), capable of detecting low mass planets, is already in operation. Next year, the Kepler satellite will be launched and will look for such planets as well. In my talk I describe the present state of our knowledge about the existence of low mass planets in extrasolar planetary systems and discuss the combined observational and theoretical efforts to find another Earth.

\section{Life, climate and the atmospheres}

\section{I Biological feedbacks as cause and demise of Neoproterozoic icehouse: implications for multicellular evolution

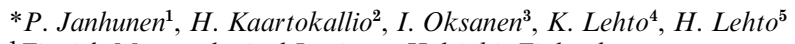 \\ ${ }^{1}$ Finnish Meteorological Institute, Helsinki, Finland \\ ${ }^{2}$ Finnish Institute of Marine Research, Helsinki, Finland \\ ${ }^{3}$ University of Helsinki, Department of Applied Chemistry and Microbiology, Helsinki, Finland \\ ${ }^{4}$ University of Turku, Department of Biology, Turku, Finland \\ ${ }^{5}$ University of Turku, Tuorla Observatory, Tuorla, and Department of Physics, Finland}

Several severe glaciations occurred during the Neoproterozoic eon, and especially near its end in the Cryogenian period (630-850 Ma). While the glacial periods themselves were probably related to the continental positions being appropriate for glaciation, the general coldness of the Neoproterozoic and Cryogenian as a whole lacks specific explanation. The Cryogenian was immediately followed by the Ediacaran biota and Cambrian Metazoa, thus understanding the climate-biosphere interactions around the Cryogenian period is central to understanding the development of complex multicellular life in general. We present a feedback mechanism between growth of eukaryotic algal phytoplankton and climate, which explains how the Earth system gradually entered the Cryogenian icehouse from the warm Mesoproterozoic greenhouse. The more abrupt termination of the Cryogenian is explained by the increase in gaseous carbon release caused by the more complex planktonic and benthic food webs and enhanced by a diversification of metazoan zooplankton and benthic animals. The increased ecosystem complexity caused a decrease in the inorganic carbon burial rate, breaking the algal-climatic feedback loop of the earlier Neoproterozoic eon. Prior to the Neoproterozoic eon, eukaryotic evolution took place in a slow timescale regulated by interior cooling of the Earth and Solar brightening. Evolution might have proceeded faster had these geophysical processes been faster. Thus, complex life could theoretically also be found around stars that are more massive than the Sun and have a main sequence life shorter than $10 \mathrm{Ga}$. We also suggest that snow and glaciers are, in a statistical sense, important markers for conditions that may possibly promote the development of complex life on extrasolar planets. 


\subsection{Life and climate: is there a link? Peter Ditlevsen}

Niels Bohr Institute, University of Copenhagen, Copenhagen, Denmark The habitability of our planet is strongly linked to the climate and existence of liquid water. The temperature on the early Earth was above the freezing point of water, despite the $30 \%$ fainter young Sun, which is probably required for development of life. The surface temperature is regulated by the amount of greenhouse warming from $\mathrm{CO}_{2}$ and possibly $\mathrm{CH}_{4}$. The atmospheric concentrations of these gases are influenced both by non-biotic geochemical processes, such as weathering of silicate rocks and by biotic processes, such as photosynthesis. These processes both act as climate regulators. From the perspective of a simple model, with substantially more realism than the Daisy world parable, it is discussed to which extent the Gaia hypothesis can be confirmed. The Climate-Biosphere system is in a dynamical steady state far from chemical and thermodynamic equilibrium. There are no known principles governing the character of this state. It has been proposed that a state of maximum entropy production could be relevant. This will be discussed in relation to the Gaia idea.

\subsection{The importance of high $\mathrm{CO}_{2}$ amounts in young terrestrial planetary atmospheres}

${ }^{*}$ H. Lammer ${ }^{1}$, M. L. Khodachenko ${ }^{1}$, M. Panchenko ${ }^{1}$, N. Terada ${ }^{2,3}$, Yu. N. Kulikov ${ }^{4}$

${ }^{1}$ Space Research Institute, Austrian Academy of Sciences, Graz, Austria

${ }^{2}$ National Institute of Information and Communications Technology,

Tokyo, Japan

${ }^{3}$ CREST, Japan Science and Technology Agency, Saitama, Japan

${ }^{4}$ Polar Geophysical Institute (PGI), Russian Academy of Sciences, Murmansk, Russian Federation

In order to understand the principles that generated Earth's long-time habitable environment compared with other terrestrial Solar System planets like Venus and Mars and terrestrial exoplanets inside the habitable zones of late-type stars, one has to understand the evolutionary influence of the Solar/stellar radiation and particle environment on the atmosphere and surface. Because the spectral type of the host star plays a major role in the photochemistry and evolution of planetary atmospheres, their water inventories must be understood within the context of the evolving stellar energy and particle fluxes. Only a stable and dense enough atmosphere, which allows water to be liquid over geological time periods and protects the planetary surface from hostile radiation, will allow the evolution of surface life and Earth-like biospheres. Atmospheric expansion and thermal and non-thermal escape depend on the stellar X-ray and EUV flux, as well as the Solar/stellar plasma flux which affects both the temperature of the upper atmosphere and photoionization processes. Observations of Solar proxies with various ages indicate that the active XUV phase of the young Sun (a representative for $\mathrm{G}$ stars) was about 6 times higher $3.5 \mathrm{Ga}$ ago. The young Solar phase included continuous flare events, and XUV radiation up to 100 times more intense than today, about $100 \mathrm{Myr}$ after the Sun arrived at the ZAMS. By using these astrophysical data one can model the influence of the evolving Solar radiation and particle environment in the upper atmospheres of Venus, Earth and Mars. Recent studies indicate that the very young Earth should have had Venus-like high values of $\mathrm{CO}_{2}$ during the first $500 \mathrm{Ma}$ after the planets origin. Such an atmosphere cooled the upper atmosphere due to IR emission and stabilized it against the high XUV flux of the young Sun. We discuss the relevance of our study for the evolution of the early Earth atmosphere and for habitability aspects of Earth-like exoplanets in orbits within habitable zones of dwarf stars.

\subsection{The life span of a photosynthetic-active biosphere on super-Earth planets \\ *C. Bounama, W. von Bloh, S. Franck}

Potsdam Institute for Climate Impact Research, Potsdam, Germany

Methods of Earth system analysis are applied to the assessment of the life span of a photosynthetic-active biosphere of super-Earth planets. Super-Earths are massive (up to 10 Earth masses) rocky planets with a chemical and mineralogical composition like Earth. They do not have significant gaseous envelopes of $\mathrm{H}_{2}$ and $\mathrm{He}$. Their water weight fraction stays below $10 \%$. The similarities in the composition of such planets with Earth allow the application of a thermal evolution model with volatile dependent rheology for whole mantle convection rescaled to super-Earth planets. With the help of a scaling law for mass and radius $\left(R \sim M^{0.27}\right)$ the thermal evolution of super-Earths is calculated und the time-dependent values of the geophysical forcing ratio (GFR) are determined. The GFR describes the influence of the volcanic activity and the continental area on the global climate in the most concise form. A hypothetical global biosphere is parameterized. The appropriate life forms feature a temperature tolerance from $0{ }^{\circ} \mathrm{C}$ to $100{ }^{\circ} \mathrm{C}$. They are able to perform photosynthesis down to a minimum $\mathrm{CO}_{2}$ partial pressure of $10 \mathrm{ppm}$. The thermal evolution of a super-Earth is linked to the luminosity evolution of its central star. Therefore, with the help of a simple climate model a given central star luminosity and $\mathrm{CO}_{2}$ concentration of the planetary atmosphere yields the surface temperature, which enters into the calculation of the productivity of the biosphere. The planet is habitable as long as the photosynthetic-active biosphere is productive. The luminosity evolution of the main-sequence central star is deduced by polynomial fitting of stellar evolution models. They also provide the nuclear time span, i.e. the time a star spends on the main sequence while burning its hydrogen fuel.

The GFR values increase with planetary mass resulting in an extended maximum life span of the biosphere $t_{\max }$. GFR values and $t_{\max }$ for a planet with a given mass are smaller as more planetary surface is covered by continents. For super-Earths, which are no pronounced land or water worlds, a power law dependence of $t_{\max }$ on planetary mass with an exponent of about 0.14 can be derived. Bigger values for $t_{\max }$ are not necessarily resulting in a longer duration of habitability. In fact the photosynthesis-sustaining habitable zone of super-Earths can be limited by the end of the main-sequence evolution of the central star. However, for less massive stars up to 0.9 Solar masses ( $\mathrm{K}$ and $\mathrm{M}$ stars) the realized life span of the parameterized photosynthetic biosphere is always determined by $t_{\max }$ and not by the nuclear time span of the host star.

\section{Mars research}

\section{I Detection of anticipated life on Mars by Phoenix}

*Joop M. Houtkooper ${ }^{1}$, Dirk Schulze-Makuch ${ }^{2}$

${ }^{1}$ Center for Psychobiology and Behavioral Medicine, Justus-Liebig-

University of Giessen, Germany

e-mail: joophoutkooper@gmail.com

${ }^{2}$ School of Earth and Environmental Sciences, Washington State

University, USA

The Phoenix lander, on its way since August, has instruments on board to explore the geology and climate of Mars in the Martian arctic and to explore if life ever arose on Mars. Here, we look at life based on, instead of water, a mixture of water and hydrogen peroxide and how this might be detected by Phoenix.

The conditions on Mars imply an evolutionary advantage for organisms employing a mixture of $\mathrm{H}_{2} \mathrm{O}_{2}$ and $\mathrm{H}_{2} \mathrm{O}$ in their intracellular fluid: the $\mathrm{H}_{2} \mathrm{O}_{2}-\mathrm{H}_{2} \mathrm{O}$ eutectic $\left(61.2 \% \mathrm{H}_{2} \mathrm{O}_{2}\right)$ has its freezing point at $-56.5^{\circ} \mathrm{C}$, but tends to supercool below that temperature. Furthermore the mixture is hygroscopic and a source of oxygen. With regard to its compatibility with cellular contents, although mostly known as a deinfectant, $\mathrm{H}_{2} \mathrm{O}_{2}$ is used for a variety of purposes in terrestrial biochemistry. 
The Viking Lander Biology Experiments have often been interpreted as the result of inorganic oxidants in the Martian soil. We interpret the Viking findings as the result of the reactions of $\mathrm{H}_{2} \mathrm{O}_{2}-\mathrm{H}_{2} \mathrm{O}$ based life (Houtkooper \& Schulze-Makuch 2007). Several hitherto puzzling findings are explained by the $\mathrm{H}_{2} \mathrm{O}_{2}-\mathrm{H}_{2} \mathrm{O}$ hypothesis. The lack of detected organics is the result of autooxidation of the organisms as these were gradually heated. The addition of water vapour at a relatively high temperature could only be withstood by the organisms for a short time, as they perished due to hyperhydration. Thus, the evolution of oxygen in the GEx experiment is explained by the high oxidative content of the organisms. Moreover, the GEx experiment allows the calculation of biomass in the Martian soil, based on measured evolution of reaction products, as 1300 ppmw.

The ability of the anticipated organisms to withstand low temperatures and the relatively high water vapour content of the atmosphere in the Martian arctic, means that Phoenix will land in an area not inimical to $\mathrm{H}_{2} \mathrm{O}_{2}-\mathrm{H}_{2} \mathrm{O}$ based life. Phoenix has a suite of instruments that may be able to detect this: The optical and atomic-force microscopes may be able to picture biological structures. Furthermore, the Thermal and Evolved Gas Analyser (TEGA) is a combination of a scanning calorimeter and mass spectrometer instrument that will analyse Martian ice and soil samples. A sample, sealed in an oven, is slowly heated at a constant rate to a temperature of $1000{ }^{\circ} \mathrm{C}$. The scanning calorimetry shows the transitions from solid to liquid to gas of the different components of the sample. The evolved gases are analysed by a mass spectrometer. For the anticipated organisms this means they decompose exothermically at a relatively low temperature compared to the endothermic reactions of the inorganic soil. This may provide a unique signature, especially in conjunction with the detection of the gaseous products, $\mathrm{CO}_{2}, \mathrm{H}_{2} \mathrm{O}, \mathrm{O}_{2}$ and $\mathrm{N}_{2}$. The isotopic composition may provide further evidence of biological origin.

Houtkooper, J. M. \& Schulze-Makuch, D. (2007). Int. J. Astrobiology 6, $147-152$

\subsection{Methane and formaldehyde: their abundance and sources on the Earth and Mars \\ ${ }^{*}$ C. Muller, D. Moreau}

Belgian Institute for Space Aeronomy and B.USOC, Brussels, Belgium Formaldehyde on earth was discovered 70 years ago in rain waters and methane was first seen as a permanent component of the Earth's atmosphere in 1948 in infrared Solar spectra recorded in Columbus, Ohio. These two components are now permanently monitored by earth observation satellites as SCIAMACHY. SCIAMACHY results will be presented and analysed in terms of biological activity on the earth.

The Martian situation is less clear, formaldehyde appears apparently in telescopic spectra obtained from the Palomar $5 \mathrm{~m}$ telescope in 1969, this observation will be described and revisited. The Russia infrared spectrometer AUGUSTE also obtained clear spectra of limb absorption by formaldehyde during the PHOBOS spacecraft mission in 1989, both these observations fail to record the presence of methane. Since 2005, methane has been recorded in nadir on Mars by the PFS interferometer on the MarsExpress spacecraft, this methane has shown spatial variations. As no chlorophyll was ever recorded on the Mars surface, the explanation of its origin is still to be decided between contemporary volcanism and a strongly active endolithic biomass.

These points will be reviewed with the assistance of the atmospheric Martian database accumulated from the entire set of Mars-Express observations.

\subsection{Geological and paleo-climatic constraints for the search of life on Mars \\ *V.-P. Kostama, M. Aittola, J. Korteniemi, H. Lahtela, T. Törmänen, T. Öhman, J. Raitala \\ Planetology Group, Astronomy Division, Department of Physical \\ Sciences, University of Oulu, Finland \\ When looking for possible locations of Martian life we study the re- gions with fluvial and glacial features to be incorporated to the future}

lander and sample return programs for closer examination. The recent Martian datasets (Zuber et al. 1992; Malin \& Edgett 2001; Christensen et al. 2004; Jaumann et al. 2007; McEwen et al. 2007) are used to study details of Martian geology and the evolution of the planet's hydrology and climate. Water was plentiful on Mars in its early history over 3.7 billion years ago, as is seen from the dendritic channel networks on the old highlands. Large channels and underwater sediments prove long-lasting widespread fluvial erosion, rivers and paleolakes (Raitala et al. 2004; Mangold \& Ansan 2005; Raitala et al. 2005). Closer to $\sim 3$ billion years ago the watery events in general became smaller. Although some recent localized formations show the effects of periodical water on the surface, most of the Martian water is now incorporated in the polar caps, permafrost, hydrated minerals, water vapour and icy particles. This is supported by our recent studies (Raitala et al. 2004; Raitala et al. 2005; Korteniemi et al. 2007; Kostama et al. 2007a,b; Lahtela et al. 2007; Raitala et al. 2007), which indicate ice accumulation on hill slope alcoves, valleylake chains, debris aprons and channel-related mesas in various regions on Mars.

Climate (Haberle 2004) has been important in repeating water accumulation and release on Mars. Within the last $10 \mathrm{Ma}$, water has been mobilized from the poles during high inclination periods of the rotation axis (now $25^{\circ}$, typical $45^{\circ}$, max. $83^{\circ}$ (Laskar \& Robutel 1993)). The insolation increase removed water from the poles and accumulated snow and ice on the mid-latitude hilltops and highlands (Head et al. 2003; Raitala et al. 2004; Head et al. 2005; Korteniemi et al. 2007; Raitala et al. 2007). The last such a period was $\sim 5.3 \mathrm{Ma}$ ago (Laskar \& Robutel 1993; Kreslavsky \& Head 2004) and several similar periods occurred $\sim 7.8-9.6 \mathrm{Ma}$ ago. The maximal pole insolation was 9000 summers long, 9.5 Ma ago (Laskar \& Robutel 1993; Kreslavsky \& Head 2004).

The reverse climate phase, decrease of inclination, in turn released water from the temporary ice reservoirs and moves it back to the poles (Kostama et al. 2006). Water release from the icy areas has subsequently eroded the surroundings and resulted in temporary channels and lakes (Raitala et al. 2005; Korteniemi et al. 2007; Kostama et al. 2007a,b; Lahtela et al. 2007; Raitala et al. 2007). An amount of water has penetrated the ground to form permafrost and groundwater leading to sapping and cavity formation (Raitala et al. 2007). This was repeated along the climate change cycle. It has resulted in frequent Martian hydrology events that provide a warmer and a more humid environment than the one observed today. In doing so, this also provides possible shelters to support the increase and evolution of life forms - if they have ever evolved on Mars.

Christensen et al. (2004). Space Sci. Rev. 110, 85-130.

Haberle (2004). LPSC 35, \#2010.

Head et al. (2003). Nature 426, 797-802.

Head et al. (2005). Nature 434, 346-351.

Jaumann et al. (2007). PSS 55, 928-952.

Korteniemi et al. (2007). Vernadsky-Brown Microsymposium 46.

Kostama et al. (2006). Geophys. Res. Lett. 33, L11201, doi:10.1029 2006GL025946.

Kostama et al. (2007a). JGR doi:10.1029/2006JE002848.

Kostama et al. (2007b). Vernadsky-Brown Microsymposium 46. Kreslavsky \& Head (2004). LPSC 35, \#1201.

Lahtela et al. (2007). Icarus (submitted).

Laskar \& Robutel (1993). Nature 361, 608-612.

Malin \& Edgett (2001). JGR 106, 23429-23570.

Mangold \& Ansan (2005). Icarus 180, 75-87.

McEwen et al. (2007). JGR 112, E05S02, doi:10.1029/2005JE002605.

Raitala et al. (2004). Vernadsky-Brown Microsymposium 40, 51.

Raitala et al. (2005). LPS XXXVI, 1307.

Raitala et al. (2007). Vernadsky-Brown Microsymposium 46.

Zuber et al. (1992). JGR 97, 7781-7797. 


\subsection{Modelling the surface and subsurface Martian} radiation environment: implications for astrobiology

${ }^{*}$ L. R. Dartnell ${ }^{1}$, L. Desorgher ${ }^{2}, J$. M. Ward ${ }^{3}$, A. J. Coates ${ }^{4}$

${ }^{1}$ CoMPLEX (Centre for Mathematics \& Physics in the Life Sciences and Experimental Biology), University College London, UK

e-mail:l.dartnell@ucl.ac.uk

${ }^{2}$ Physikalisches Institut, University of Bern, Switzerland

${ }^{3}$ Department of Biochemistry and Molecular Biology, University College London, UK

${ }^{4}$ Mullard Space Science Laboratory, University College London, UK

The warm wet primordial Martian environmental conditions support the prospect of the development of native life. The damaging effect of ionising radiation is one of the prime limiting factors on the survival of life in such potential astrobiological habitats. Surface life on Earth is protected from Solar and cosmic ionising radiation by a global dipole magnetic field and thick atmospheric column. Whereas primordial Mars is believed to have possessed a dense carbon dioxide atmosphere, and there is evidence from crustal magnetic anomalies that Mars had a global dipole around 4 billion years ago, present day Mars receives little shielding from cosmic radiation. The Martian topsoil is thought to have been rendered completely sterile by oxidising conditions created by UV radiation, but the penetration of energetic ionising particles, which are extremely damaging to life, exceeds this depth. We investigate the extent to which the Martian present and past atmosphere and various surface scenarios attenuate the radiation environment and the potential effect on the survival of life.

We have built a computer model of radiation penetration using Geant4, a simulation toolkit for particle physics. This Monte Carlo model tracks the propagation of primary particles, and the generated secondary cascades, through both the Martian atmosphere and three different surface scenarios (dry regolith, water ice, and regolith with layered permafrost) in order to calculate the particle spectra and microbial absorbed radiation dose as a function of depth underground. Both galactic cosmic rays (GCR) and Solar energetic particles (SEP) over a wide energy range are considered. The persistence times of metabolically dormant cells or spores at different depths can then be calculated. Such analysis will be invaluable in the planning of samplereturn missions. We will report results relevant to the question of planetary protection, life held dormant in regolith permafrost, and the possibility of deep subsurface life cryopreserved in the putative Elysium pack-ice.

\section{Early Life}

\section{I The origin and evolution of viruses}

D. H. Bamford

Institute of Biotechnology and Department of Biological and

Environmental Sciences, Biocenter 2, PO Box 56, University of Helsinki, Finland

Viruses are the most abundant living entities in the biosphere outnumbering their host organisms by one to two orders of magnitude. It is conceivable that they cause the highest selective pressure their hosts encounter. As obligate parasites viruses are dependent on their hosts but their origins seem to deviate from that of cellular life.

What are the possible principles to build viruses is an open question. However, structural studies on virus capsids and coat proteins propose that there are only a limited number of ways to construct a viral protein coat. Consequently, relatedness of viruses is not connected to the type of cells they infect and the same architectural principle of the capsid has been observed in viruses infecting bacteria as well as humans. Using the viral capsid structure it is possible to group viruses to several lineages that may have existed before the three cellular domains of life (bacteria, archaea and eukarya) were separated. This would mean that viruses are ancient and that early cells were infected with many viruses proposing that the origin of viruses is polyphyletic opposing the monophyletic origin of cellular life.

\subsection{Horizontal gene transfer: evolution by default or product of natural selection? Anthony Poole \\ Department of Molecular Biology \& Functional Genomics, Stockholm} University, Stockholm, Sweden

Horizontal gene transfer describes a broad range of processes by which genes may move between genomes. The realization that transfer is common between microbes has led to much speculation concerning its role in early evolution. In particular, it has been proposed that transfer may have been so extensive that it dominated vertical inheritance (the process by which genes are transferred from parent to offspring). This view considers horizontal gene transfer as a default state in biological systems, and that its origins do not require special explanation. I will argue against this standpoint, and propose that horizontal gene transfer is a product of natural selection. Certain genes may be lost from the genomes of species where environmental conditions are constantly in flux. The emergence of horizontal transfer is, in my opinion, a strategy that aids gene survival in the face of such loss, and is best understood by considering the selective pressures that have shaped bacterial and archaeal genomes.

\subsection{Membranes and the origin of cellular life \\ * Tiina Laiterä, Kirsi Lehto \\ Department of Biology, University of Turku, Turku, Finland}

Membranes are a crucial feature to modern cellular life. Modern membranes have complex structures and functions, assisted by different kinds of protein complexes, but in early life the primary function of membranes was most likely the formation of enclosed compartments for primitive cell chemistry to take place. Prebiotic reactions leading to chemical and biological evolution - and to life - would have never been possible in dilute surroundings of the Primordial Ocean, without some means for the concentration and accumulation of molecules and substrates. Due to the crucial nature of membranes in the origin of life many scientists debate the origin of membranes in the first place. Many theories of the very first membrane components have been presented, including aromatic hydrocarbons instead of lipid-like molecules, which are used in modern cells.

Here we will introduce different theories on the origin of the first membrane components, ranging from extraterrestrial origins to random pre-biotic chemistry, and on the nature of these components, ranging from non-carbon-based alternatives to aromatic hydrocarbons. The early assembly of simple lipid molecules due to wave actions of primordial shores will be also considered. Alternative theories will be given on the evolution of membrane vesicles, formed of simple source materials, to highly sophisticated and autonomous cellular systems: what events in the early life could have led primitive membranes to evolve at a rapid pace, what changes could have enabled the appearance of more and more complex structures, and how the primitive cell, as a mere encapsulated vesicle, could have performed primitive metabolism to create energy for its functions. The question is not just about the factors and conditions leading to the appearance of the first membrane vesicles, but also the nature of those membranes. What kind of actions did the very first membranes perform? How did they begin to interact with some primitive replicating molecules and other membrane vesicles? Are the modern viruses relicts of early life left behind from some developmental point between the vesicle and the first actual cell? 
4.4 Elaborate morphologies in probable microfossils from Archaean chert, Pilbara Craton, Western Australia: indicators of complex cell behaviour? ${ }^{*}$ K. Sugitani ${ }^{1}, K$. Grey ${ }^{2,3}$, T. Nagaoka ${ }^{4}, K$. Mimura ${ }^{1}$, M. R. Walter ${ }^{3}$ ${ }^{1}$ Graduate School of Environmental Studies, Nagoya University, Nagoya 464-8601, Japan

${ }^{2}$ Geological Survey of Western Australia, Department of Industry and Resources, 100 Plain Street, East Perth, WA6004, Australia

${ }^{3}$ Australian Centre for Astrobiology, Macquarie University, Sydney, NSW 2109, Australia

${ }^{4}$ School of Informatics and Sciences, Nagoya University, Nagoya 4648601, Japan

In the greater than ca. 2.97 Ga Farrel Quartzite (Gorge Creek Group) at Mount Grant and Mount Goldsworthy, Pilbara Craton, Western Australia, a diverse assemblage of highly probable microfossils is present (Sugitani et al. in press). Four major morphological types have been preliminarily identified: thread-like, film-like, spheroidal and lenticular to spindle-like, each of which can be further subdivided into several sub-types. Biogenicity of most of the microstructures can be inferred from indigenousness, syngenecity, the shallow sedimentary origin of the host chert, relatively narrow size distribution, composition (disordered carbon), evidence of flexible and/or breakable walls, apparent taphonomic features and the presence of colony-like aggregations.
Morphological variability suggests that a diverse microbial ecosystem flourished in the Pilbara region during the Archaean.

In addition to the above morphological types, several highly elaborate, complex morphologies were found. They are include (1) pairs of attached spheroids, (2) chain-like arrangements of multiple lenticular structures, (3) oblique arrangement of two lenticular structures, (4) a spheroid with a smaller bud-like appendage, and (5) large spheroids and spindles containing internal objects. These 'composite' morphologies are suggestive of colonial and reproductive styles observed in modern microorganisms. If some of the Farrel Quartzite microstructures can be shown to represent reproducing cells, this coupled with the unusually large size for prokaryotic microorganisms (spheroidal and spindle-like units are occasionally up to $30 \mu \mathrm{m}$ or more in size), would have major implications for evolution and indicate considerable biotic diversity by the late Archean. Based on the examination of more than 2000 specimens, we examine a working hypothesis that some Farrel Quartzite microstructures represent reproductive structures, providing a framework on which to base for future taxonomic and phylogenic studies.

Sugitani et al. (in press). Diverse microstructures from Archaean chert from the Mount Goldsworthy-Mount Grant area, Pilbara Craton, Western Australia: Microfossils, dubiofossils, or pseudofossils? Precambrian Res.

\section{Prebiotic chemistry}

\section{I Conformation-dependent racemization of aspartyl residues in model peptides \\ *A. Brack ${ }^{1}$, K. Kuge ${ }^{2}$, N. Fujii ${ }^{2}$ \\ ${ }^{1}$ Centre de biophysique moléculaire, CNRS, Orléans, France \\ ${ }^{2}$ Research Reactor Institute, Kyoto University, Osaka, Japan}

Peptides were expected to be protected against racemization in betasheet structures because the beta-sheet cannot accommodate heterochiral chains containing both $\mathrm{L}$ and $\mathrm{D}$ amino acids. In such a structure, the side-chains point perpendicular to the plane of the sheet and the hydrogen atoms lie almost in the plane. Stereoinversion, i.e. the replacement of some L-enantiomers by D ones, would force the sidechains of the D-residues to lie in the plane of the sheet, a situation that has been shown to be impossible. When increasing amounts of Denantiomers are introduced in a beta-sheet forming all-L peptides, only those segments having seven adjacent residues of identical chirality can associate to form beta-sheet nuclei surrounded by random coil heterochiral segments. In contrast, peptides with alpha-helical structures are capable of accommodating both $\mathrm{L}$ and $\mathrm{D}$ amino acids. Kinetic analysis of racemization rates of Asp residues in all-L (Leu-Asp-Asp-Leu)8-Asp and in all-L (Asp-Leu) 15 were run to investigate the influence of secondary structures on the racemization of Asp residues. The two peptides, (Leu-Asp-Asp-Leu)8-Asp and (Asp-Leu)15, have almost the same number of Asp residues but they adopt different chain conformations. The two peptides adopt a random coil conformation in pure water due to charge repulsion but the addition of low concentrations of $\mathrm{Zn}++$ cations specifically induces an alpha-helix structure for (Leu-Asp-Asp-Leu)8-Asp and a beta-sheet structure for (Asp-Leu)15. The kinetic data showed that the activation energy of racemization of Asp residues in beta-sheets of (Asp-Leu)15 is higher than that in alpha-helicoidal (Leu-Asp-Asp-Leu)8-Asp. Furthermore, at $37^{\circ} \mathrm{C}$, the time required for the $\mathrm{D} / \mathrm{L}$ ratios of Asp residues in (Asp-Leu) 15 and (Leu-Asp-Asp-Leu)8-Asp to approximate 1.0 were estimated at 123 and 13 years, respectively. These results indicate that Asp residues are protected against racemization in beta-sheet structures.

\subsection{Driving chirality}

Harry J. Lehto

Tuorla Observatory and Department of Physics, University of Turku, Finland

Life differs from the non-living in the respect that it uses specific chirality. All amino acids in proteins are of the L-configuration, and the sugars on the other hand are all of D-configuration. The source of chirality is still an enigma. A natural driving force for favouring one enantiomer over the other could be exposure to circularly polarized radiation (CPR). There have been several attempts in trying to explain CPR as of stellar origin, but the total flux of CPR from stellar light appears to be much too low.

A local source of CPR would be very beneficial in forcing selection towards a single chirality. This could have happened on the young Earth planet in a combination of an air-liquid-solid interface. When light strikes the surface of liquid at an angle, part of the light is reflected back as in a mirror. Part of the light is refracted into the liquid. The reflected light is linearly polarized in a 'horizontal' direction. The refracted light ray entering the liquid has a strong component, which is linearly polarized in a direction normal to the horizontal and the propagation direction of the refracted ray.

Linearly polarized light is not quite what we need. We would require a medium, which effectively works as a retarder or a 'quarter wave plate'. This could change the linearly polarized light into a circularly polarized one and cause a local excess of one CPR component.

The advantage of this system is that sunlight would be the original light source and the polarization would be produced locally. It would be available in relatively large quantities for extended periods and be generally present in any planet.

We will discuss the properties of possible retarders, effects of Solar motion on the sky, and the diffuse scattered polarization of the sky. We will also hypothesize about where on the Earth could this happen and what would be the consequences. 


\subsection{Emergence of chirality and molecular evolution \\ * Raphaël Plasson ${ }^{1,2}$, Hugues Bersini ${ }^{2}$, Axel Brandenburg ${ }^{1}$ \\ ${ }^{1}$ NORDITA, Stockholm, Sweden \\ ${ }^{2}$ IRIDIA, Université Libre de Bruxelles, Belgium}

Since Frank's article (Frank 1953), several far-from-equilibrium models where developed, investigating the spontaneous onset of a nonracemic state from a symmetrical system. They are generally based on open-flow systems, incorporating autocatalytic reactions. However, recent works show how such models can be improved by replacing open-flows by activated recycling of compounds, and by allowing emergent network-autocatalytic behaviours rather than direct autocatalytic reactions (Plasson et al. 2004, 2007). This approach leads to the description of non-equilibrium chemical systems as subsets of direct matter fluxes and simple reactions cycles. A formalism can be developed on the basis of the stoichiometric matrix of the reaction network, generalizing the formalism used in the literature to describe the metabolisms of alive beings. The coupling of this description with kinetic and thermodynamic parameters allows the description of evolutionary chemical networks. In this framework, homochirality doesn't appear as a strange event in the process of the origin of life, but is rather only one element that can be formed by the first emerging proto-metabolisms, potentially allowing further complexity. Such proto-metabolic patterns can be compared to Eigen's hypercycles (Eigen et al. 1977). They may have constituted the first replicator, not as template replicators of similar molecules, but allowing the replication of the hypercycles themselves as elementary objects, allowing bifurcation towards some, and extinction of others. Such a description leads to a hierarchical view of the emergence of life, from synergy between simple reactions producing the emergence of proto-metabolisms, to synergy between protometabolism producing the emergence of metabolisms, evolving with increasing complexity towards replicative molecules.

Eigen, M. \& Schuster, P. (1977). Naturwiss. 64, 541-565.

Frank, F. C. (1953). Biochem. Biophys. Acta 11, 459-463.

Plasson, R., Bersini, H. \& Commeyras A. (2004). Proc. Natl. Acad. Sci. USA 101, 16733-16738.

Plasson, R., Kondepudi, D. K., Bersini, H., Commeyras, A. \& Asakura, K. (2007). Chirality 19, 589-600.

\subsection{A terrestrial prebiotic route to pyrroles and higher aromatics}

*H. Strasdeit, S. Fox, D. Denysenko

Department of Bioinorganic Chemistry, Institute of Chemistry,

University of Hohenheim, 70599 Stuttgart, Germany

In the Hadean and early Archean, hot volcanic coasts probably occurred more frequently than today. At these locations, seawater must have formed salt crusts that often experienced temperatures of up to several hundred degrees Celsius. Organic compounds dissolved in the ocean were embedded in these crusts.

We have modelled this scenario by evaporating artificial seawater containing $\mathrm{NaCl}, \mathrm{KCl}, \mathrm{MgCl}_{2}$, and $\mathrm{CaCl}_{2}$, and low concentrations of one of the a-amino acids glycine, rac-alanine, rac-valine, rac-glutamic acid, rac-isovaline, and a-aminoisobutyric acid. The solid salt mixtures obtained were thermolized at $350^{\circ} \mathrm{C}$ in a slow stream of nitrogen. In all cases, except for glycine, $1 \mathrm{H}$-pyrroles were detected among the products. For rac-alanine (Hala) we found that under our experimental conditions neither the neat amino acid nor solid $\mathrm{NaCl} / \mathrm{KCl} / \mathrm{Hala}$ mixtures produced pyrroles on heating. In fact, $\mathrm{CaCl}_{2}$ plays a key role: we were able to show, by means of X-ray crystallography and vibrational spectroscopy, that solid artificial sea salt-alanine mixtures contain the previously unknown coordination compound $\mathrm{Ca}_{3} \mathrm{Cl}_{6}(\mathrm{Hala})_{2} 6 \mathrm{H}_{2} \mathrm{O}$ (1). Pure 1 could be prepared on a multi-gram scale.

GC/MS analyses showed that alkylated 1H-pyrroles (2) are by far the most abundant volatile thermolysis products obtained from 1 at $350{ }^{\circ} \mathrm{C} .31$ different methylated and ethylated pyrroles, up to tetraethyl$1 \mathrm{H}$-pyrrole, have been identified.

Pyrroles are formed over a wide temperature range of $300{ }^{\circ} \mathrm{C}$ to $800^{\circ} \mathrm{C}$. At $800^{\circ} \mathrm{C}$, several other compounds occur which are not observed at the lower end of the temperature range. Benzo-nitrile, styrene, indene, indole (3), naphthalene, quinoline, isoquinoline (4), acenaphthylene (5), fluorene, anthracene, phenanthrene (6), fluoranthene (7), and pyrene (8) were positively identified. The formation of pyrroles and polycyclic aromatic hydrocarbons (PAHs) is not restricted to sea salt-amino acid mixtures but also occurs when Ca-montmorillonite-amino acid intercalates are heated. The prebiotic thermolysis of a-amino acids was possibly a first step towards oligopyrroles such as porphyrins. It may also have opened a doorway into a ' $\mathrm{PAH}$ world' (Hazen 2005) or 'aromatic world' (Ehrenfreund et al. 2006).

Acknowledgment: We thank the Deutsche Forschungsgemeinschaft for financial support.

Ehrenfreund, P., Rasmussen, S., Cleaves, J. \& Chen, L. (2006). Experimentally tracing the key steps in the origin of life: the aromatic world. Astrobiology 6, 490-520.

Hazen, R. M. (2005). Genesis: The Scientific Quest for Life's Origin, ch. 17. Joseph Henry Press, Washington, DC.

\subsection{Oxidative pathways of reduced phosphorus compounds: a primary source of condensed phosphates on the early Earth \\ ${ }^{*}$ M. A. Pasek ${ }^{1}$, T. P. Kee ${ }^{2}$, J. I. Lunine ${ }^{1}$ \\ ${ }^{1}$ NASA Astrobiology Institute, University of Arizona, 1629 E. University Blvd., Tucson, AZ 85721, USA \\ e-mail:mpasek@lpl.arizona.edu \\ ${ }^{2}$ School of Chemistry, University of Leeds, Woodhouse Lane, Leeds LS2 9JT, UK}

Phosphorylated biomolecules like RNA, DNA, ATP, phospholipids, and many coenzymes are critical to life as we know it. Presumably, given their central role in life, phosphorylated biomolecules were also critical for the origin or early evolution of life on the Earth. The origin of these biomolecules remains one of the major questions in origins of life research. Recent discoveries by our group (Pasek et al. 2005, 2007) and by the Leeds group (Bryant \& Kee 2006) have suggested that the reaction of meteoritic schreibersite with water produces abundant reduced phosphorus compounds, which were both more soluble and chemically reactive than terrestrial phosphates. We discuss here the products resulting from the oxidation of phosphite (H-phosphonate) and hypophosphite (H-phosphinate) in aqueous solution. Phosphite oxidizes to orthophosphate, but also forms a significant quantity of pyrophosphate $(5-15 \%)$, and triphosphate $(0.1-1 \%)$. The relevance of these pathways to the origin and evolution of early life is discussed. Bryant, D. E. \& Kee, T. P. (2006). Chem. Commun. 22, 2344. Pasek, M. A. \& Lauretta, D. S. (2005). Astrobiology 5, 515.

Pasek, M. A., Dworkin, J. P. \& Lauretta, D. S. (2007). Geochim. Cosmochim. Acta 71, 1721

\subsection{Phosphorus redox chemistry in planetary environments: implications for the origins of life} D. E. Bryant ${ }^{1}$, I. B. Gorrell ${ }^{1}$, D. Greenfield ${ }^{2}$, S. M. Evans ${ }^{1}$, L. Wang ${ }^{3}$, D. E. Heard ${ }^{1}$, M. A. Blitz ${ }^{1}$, A. Goddard ${ }^{1}$ C. Smith ${ }^{4}$, M. A. Pasek, *T. P. Kee ${ }^{1}$

${ }^{1}$ School of Chemistry, University of Leeds, Woodhouse Lane, Leeds LS2 9JT, UK

e-mail:t.p.kee@leeds.ac.uk

${ }^{2}$ Centre for Corrosion Technology, Materials and Engineering Research Institute, Sheffield Hallam University, Sheffield S1 $1 \mathrm{WB}, U K$

${ }^{3}$ School of Chemistry, South China University of Technology, Guangzhou, 510640, China

${ }^{4}$ Department of Mineralogy, The Natural History Museum, Cromwell Road, London SW7 5BD, UK

${ }^{5}$ NASA Astrobiology Institute, University of Arizona, 1629 E. University Blvd., Tucson, AZ 85721, USA

On Earth, phosphorus occurs naturally as fully oxidized orthophosphate $\left(\mathrm{PO}_{4}{ }^{3-}\right)$ most commonly within igneous and metamorphic rocks as the apatite family $\mathrm{Ca}_{5}\left(\mathrm{PO}_{4}\right)^{3}(X)[X=\mathrm{Cl}, \mathrm{F}, \mathrm{OH}]$. However, recent discoveries by Pasek \& Lauretta in Arizona (Pasek \& Lauretta 2005) and in our own laboratory (Bryant \& Kee 2006) suggest that chemical modification of schreibersite inclusions (Fig. 1) within iron 
meteorites may have provided a source of phosphorus both significantly more water-soluble and chemically reactive than orthophosphate. Here we discuss a range of reactive phosphorus products including $\mathrm{H}$-phosphinic acid $\left(\mathrm{H}_{3} \mathrm{PO}_{2}\right)$ and phosphine $\left(\mathrm{PH}_{3}\right)$ that result from chemical modification of meteoritic phosphide inclusions under anaerobic photochemical, Galvanic and microwave plasma conditions. We report also the first application of the scanning electrochemical techniques such as SVET (Fig. 2) to explore corrosion on meteorites and discuss some potential consequences for prebiotic chemistry.

Pasek, M. A. \& Lauretta, D. S. (2005). Astrobiology 5, 515.

Pasek, M. A., Dworkin, J. P. \& Lauretta, D. S. (2007). Geochim. Cosmochim. Acta 71, 1721.

Bryant, D. E. \& Kee, T. P. (2006). Chem. Commun. 22, 2344.

\subsection{Abiotic organic synthesis underneath the ocean floor \\ Nils G. Holm \\ Department of Geology and Geochemistry, Stockholm University,} Stockholm, Sweden

The formose reaction has been outdated for abiotic synthesis of carbohydrates for a while. However, it is still possible that it is responsible for prebiotic formation of ribose in natural environments and that this may occur in close vicinity to purine synthesis and phosphorylation processes in hydrothermal environments of convergent margins. The potential of pyrophosphate formation upon heating of hydrogenated orthophosphates to a few hundred ${ }^{\circ} \mathrm{C}$ in geological environments where the activity of water is low has probably been underestimated. Once pyrophosphate is available, phosphorylation of ribose and/or nucleosides may occur. Boron is an element that has a strong affinity for organic material since it forms trigonal and tetrahedral complexes with oxygen groups. For instance, boric acid and borate readily form complexes with a wide variety of sugars - pentoses such as ribose, in particular - and other compounds containing cis-hydroxyl groups (humic substances). The awareness today that ribose is stabilized by borate has resulted in a metamorphosis of the formose reaction from a seemingly random and non-selective reaction into a very precise pre-RNA process.

\section{Small bodies in the Solar System: Comets, Meteorites, Moons}

\section{I Unbiased meteorite statistics: an impossible} task? The Oman experience

*Beda A. Hofmann ${ }^{1}$,Edwin Gnos ${ }^{2}$, Manuel Eggimann ${ }^{1,3}$, Ali Al-Kathir ${ }^{4}$ ${ }^{1}$ Natural History Museum Bern, Bernastrasse 15, CH-3005 Bern

${ }^{2}$ Musée d'histoire naturelle de la Ville de Genève, 1 route de Malagnou, CP 6434, CH-1211 Geneva, Switzerland

${ }^{3}$ Institute for Geological Sciences, Baltzerstrasse 1-3, CH-3012 Bern

${ }^{4}$ Ministry of Commerce and Industry, Salalah, Sultanate of Oman

Meteorites are dominantly asteroidal samples delivered to earth 'for free'. Knowledge of the relative contribution of different types of meteorites to this flux is of great interest because it reveals information about the relative abundance of different types of asteroids. However, statistical results obtained on a dataset including all meteorite finds are not representative for the composition of the flux, because certain types (e.g. irons) are more easily recognized, especially in temperate climates. Collections from deserts suffer from usually unresolved pairing of strewnfields. The same effect probably applies to the Antarctic meteorite collections as well. Nullarbor (Australia) and Antarctic collections show mass distributions with median masses around $0.01 \mathrm{~kg}$ (Bevan et al. 2001). The roughly 1000 observed falls are commonly referred to as the most representative sample, but this is also biased due to an over-representation of large meteorites (median mass $3.0 \mathrm{~kg}$ ). We have used the Omani-Swiss collection recovered during six search campaigns (2001-2007) to obtain a meteorite compositional statistic for the Omani desert. This statistic is based on 4985 meteorite samples (3800 kg mass). We have checked all our Oman samples for pairing based on find locations and petrography by direct comparison of thin sections. This sample represents the only large group of meteorites for which a correction for pairing has been made by direct comparison of meteorites. Pairing groups range from large strewnfields (JaH 073: 3000 stones, $600 \mathrm{~kg}$; JaH 091: 600 stones, $3 \mathrm{t}$ mass) to medium strewnfields (tens of stones) to few paired stones. After consideration of all pairings, our Oman sample represents $300-350$ fall events. An interesting result of this effort is a pairing-corrected ratio of 1.6 for $\mathrm{H} / \mathrm{L}$ ordinary chondrites, similar to values obtained for Antarctica and the Sahara, while recent falls show a lower ratio of 0.9 (Bevan et al. 2001). $\mathrm{The} \mathrm{H} / \mathrm{L}$ ratio is unlikely to be affected by the various sources of bias. Carbonaceous/anomalous chondrites only represent $2 \%$ of finds $(5 \%$ of falls). Our results indicate that recent falls represent only a short time average, while the flux may be subject to significant changes over several tens of thousands of years.

We recognize that our Oman sample also is biased due to: (1) possible under-representation of small meteorites due to their lower visibility, and due to disintegration; (2) near-lack of iron meteorites due to sinking into soil and/or ancient collecting; (3) differential weathering of meteorite types. However, the exact knowledge of all fall locations, the low median mass (compared with recent falls) and the availability of samples for direct comparison probable render the Oman sample one of the statistically soundest. Further comparisons with observed falls will also consider mass-abundance relations for each meteorite type.

Bevan, A. W. R., Bland, P. A. \& Jull, A. J. T. (2001). Meteorites: Flux with time and impact effects, ed. Grady, M. M., Hutchison, R., McCall, G. J. H. \& Rothery, D. A., p. 59. Geological Society, London.

\subsection{Organics on Wild 2 comet: laboratory results of stardust samples \\ *J. R. Brucato and The Stardust Team \\ INAF-Osservatorio Astronomico di Capodimonte, Napoli, Italy}

We report combined micro-infrared, micro-Raman, and Field Emission Scanning Electron Microscope analyses of particles collected by the Stardust spacecraft during its fly-by of comet $81 \mathrm{P} /$ Wild 2 on 2 January 2004 and successfully returned back to Earth on 15 January 2006. We present data acquired from 16 particles. These data indicate that cometary grains are rich in various and complex organic compounds and show compositional and structural heterogeneities. The aliphatic content indicates the presence of longer or less branched aliphatic chains that those observed in the interstellar medium. A comparison with micro-Raman spectra obtained from interplanetary dust particles (IDPs) and meteorites yields that the cometary carbonaceous material spans a range of processing similar to IDPs and the most primitive meteorites, indicating the presence of highly 'primitive' materials. Low-mass polyciclic aromartic hydrocarbons (PAHs) are also detected. Both the IR and Raman data imply very labile carbonaceous components, mixed with refractory minerals, which have not been observed in meteorites and IDPs. Moreover, hydrated minerals may be present in two particles one of which may also contain carbonates. 


\subsection{Composition of Saturnian E-ring Particles. Probing subsurface Oceans of Enceladus?}

*F. Postberg ${ }^{1}$, S. Kempf ${ }^{1,2}$, R. Srama ${ }^{1}$, E. GRü̈N ${ }^{1}, J$. K. Hillier ${ }^{3}$, S. F. Green ${ }^{3}$, N. McBride ${ }^{3}$

${ }^{1}$ MPI für Kernphysik, Heidelberg, Germany

${ }^{2}$ Institut für Geophysik und extraterrestrische Physik, Universität Braunschweig, Germany

${ }^{3}$ Planetary and Space Science Research Institute, The Open University, Milton Keynes, UK

The population of Saturn's outermost tenuous E-ring is dominated by tiny water ice particles. Active cryo volcanism on the moon Enceladus, embedded in the E-ring, has been known to be a major source of particles replenishing the ring since late 2005. Therefore, particles in the vicinity of Enceladus provide crucial information about the dynamic and chemical processes occurring far below the moon's icy surface. We present a compositional analysis of thousands of impact ionization mass spectra of Saturn's E-ring particles, with sizes predominantly below $1 \mu \mathrm{m}$, detected by the Cosmic Dust Analyser onboard the Cassini spacecraft. Our findings imply that organic compounds are a significant component of icy particles ejected by Enceladus plumes. Our in situ measurements are supported by detections of other Cassini instruments and hint at a dynamic interaction of a hot rocky core with liquid water at one of the candidates for a habitat in the Solar System.

\subsection{Interplay of novel organic and inorganic chemistry on Titan \\ Sam Abbas \\ El Paso Community College, Val Verde Campus, Texas, USA}

Titan's atmospheric and surface environments house complex hydrocarbon and nitrogen chemistry that may lead to the production of biologically-significant molecules such as amino acids and nitrogenous bases (Abbas et al. 2006). Data obtained from the Cassini-Huygens mission indicates a heterogeneous setting that may contain certain microenvironments of biological interest. Here we assert the notion that plausible organic synthesis that may lead to the production of biologically-important molecules is limited to restricted microenvironments that are the site of several constraints under Titan's conditions (Abbas et al. 2007). These microenvironments are limited to bounded interfaces that make use of energy gradients generated by instant heating as may be provided by meteoritic impacts in an otherwise kinetically-slow environment. Sources of energy that may drive organic synthesis include atmospheric convective currents as well as convection from the interior, chemical potentials at the interfaces, and free energy from reactions and low entropy process. A picture of Titan's near surface environment illustrating the collaboration of these effects in such microenvironments will be drawn. The interaction between inorganic ingredients and their contribution to organic synthesis in such microenvironments will be evaluated and the relevance of such chemistries to the overall progress of prebiotic evolution on Titan will be discussed.
Abbas, S., Ezzeddine, R., Lannutti, N. \& Schulze-Makuch, D. (2006). Synthesis of biologically-important precursors from nitrogencontaining species on Titan. Proc. 6th European Workshop on Astrobiology, Lyon, France, 16-18 October, 2006.

Abbas, S., Lannutti, N. \& Schulze-Makuch, D. (2007). The possibility of life in microenvironments on Titan. Poster Presentation at the Sackler Colloquium of the National Academy of Sciences, Irvine, CA, 5-6 January, 2007.

\subsection{Photochemistry of organic molecules related to Mars, Titan, meteorites and comets: experiments in terrestrial orbit and laboratory simulations}

*Y. Y. Guan 1 , H. Cottin 1 , P. Coln ${ }^{1}$, D. Coscia 1, N. Fray ${ }^{1}$, F. Macari ${ }^{1}$, F. Stalport ${ }^{1}$, F. Raulin ${ }^{1}$, C. Szopa ${ }^{2}$, D. Chaput ${ }^{3}$, M. Viso ${ }^{4}$, M.

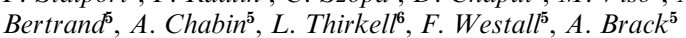

${ }^{1}$ LISA, Université Paris 7 \& Paris 12, UMR 7583 CNRS, Avenue du Général de Gaulle, 94010 CRETEIL cedex, France

${ }^{2}$ Service d'aéronomie, UMR 7620 CNRS, Réduit de Verrières - BP 3, Route des Gatines, 91371 Verrières le Buisson Cedex, France, France ${ }^{3}$ CNES, Centre spatial de Toulouse, 18 avenue Edouard Belin, 31401 Toulouse Cedex 9, France

${ }^{4}$ CNES, 2 place Maurice Quentin, 75039 Paris Cedex 01, France

${ }^{5} C B M, C N R S$, Rue Charles-Sadron, 45071 Orleans Cedex 2, France

${ }^{6}$ LPCE, CNRS, Avenue de la Recherche Scientifique, 45071 Orleans

Cedex 2, France

Photochemistry is leading the chemical evolution in the Solar System. The VUV photolysis of organic compounds is easy to study in the laboratory, with monochromatic sources, but it is difficult to simulate the whole range of wavelengths corresponding to the most energetic part of the Solar radiation $(<190 \mathrm{~nm})$. This is why the results obtained in the laboratory are difficult to extrapolate to the extraterrestrial environments. Space is the only laboratory allowing the exposure of samples to all the space parameters simultaneously.

We present the preparation and follow-up of experiments with exobiological interest, in Terrestrial orbit (AMINO, PROCESS, and UV-olution), organized by the European Space Agency (ESA). The experiments are carried out on a FOTON capsule, using the BIOPAN facility (UV-olution September 2007), and on the International Space Station, using the EXPOSE facilities (AMINO, PROCESS 2008-2009). Firstly, we will present the development of the protocols for the preparation of thin layers of molecules with exobiological interest (relative to Mars, Titan, meteorites and comets). These organic deposits are carried out on windows made of Quartz or $\mathrm{MgF}_{2}$, which constitutes our samples. These samples will remain in space between 10 days and 18 months (according to the experiment). The evolution of the samples can be studied when the samples return to Earth. These analyses will be supplemented by simulations of irradiation in the laboratory in order to calculate kinetic constants and products of these reactions. This work should contribute to the better understanding of the evolution of organic matter in the extraterrestrial environments and their potential implication in exobiology. Preliminary results of the Biopan Experiment will be presented. 


\section{Life in extreme conditions}

7.I Microbial life in extreme subglacial Antarctic lake environments: Lake Vostok

${ }^{*}$ S. Bulat ${ }^{1,5}$, I. Alekhina ${ }^{1,5}$, V. Lipenkov ${ }^{2}$, V. Lukin ${ }^{2}$, D. Marie ${ }^{3}$, C. Lavire ${ }^{4}$, P. Normand ${ }^{4}, J$. R. Petit ${ }^{5}$

${ }^{1}$ Petersburg Nuclear Physics Institute RAS, St. Petersburg-Gatchina, Russia

e-mail:sergey.bulat@ujf-grenoble.fr

${ }^{2}$ Arctic and Antarctic Research Institute, St. Petersburg, Russia

${ }^{3}$ Station Biologique de Roscoff, Roscoff, France

${ }^{4}$ Ecologie Microbienne, UMR-CNRS 5557, Université Claude BernardLyon I, France

${ }^{5}$ Laboratory of Glaciology and Geophysics of Environment CNRS, Grenoble, France

Since subglacial Antarctic lake environments (SALE) are thought to be analogues for extraterrestrial environments such as moon Europa of Jupiter, the ability of these environments to support microbial life is hotly discussed because of the high chance of 'forwardcontamination', which necessitates caution when interpreting the results.

The objective of the study was searching for DNA signatures in accretion ice from Lake Vostok buried beneath the $4 \mathrm{~km}$ thick East Antarctic ice sheet. The ultimate goal was discovering microbial life in this extreme icy environment featuring $\sim 15 \mathrm{Ma}$ long isolation, no light, high pressure (about 400 bars), low temperature (close to the freezing point), ultra-low dissolved organic carbon (DOC) content, and predicted excess of dissolved oxygen.

The 16S ribosomal RNA gene sequencing constrained by Ancient DNA research criteria of authentication was used as a main approach. The flow cytometry was used for cell enumeration.

The accretion ice retrieved by deep ice coring at Vostok provides the best present-day template for searching for life in SALE. This ice consists of two layers: the uppermost ice (type 1) containing small micaclay inclusions and the deeper ice (type 2) which is just very clean. Both ice types feature ultra-low total gas (2-3 order of magnitude lower than in glacier ice) (anaerobic conditions) and ultra-low DOC content $(<10$ ppbC) (ultra-oligotrophic conditions). The possible redox couples capable of supporting chemoautotrophic life seem to be limited to hydrogen (and possibly sulphides) as reducers and sulphates and carbon dioxide as electron acceptors and carbon source. Therefore, in accretion ice (namely ice 1) we could discover anaerobic chemoautotrophic piezophilic psychrophiles while in the water body (supersaturated with oxygen) - the same forms but 'oxygenophilic' or aerobic.

However, the DNA study showed that accretion ice in general is essentially microbial DNA-free. Up to now, a few bacterial phylotypes have been recovered from accretion ice type 1 only and they will be discussed in terms of the unusual geobiochemical environment of the lake. Amongst them the only chemolithoautotroph proved to be the true thermophile Hydrogenophilus thermoluteolus. It was initially found at $3607 \mathrm{~m}$ depth but later confirmed for another ice horizon $(3561 \mathrm{~m})$, which differed by $47 \mathrm{~m}$ core depth and about $5000 \mathrm{yr}$ time-period. The thermophiles seem to originate not from the lake water but rather within the lake bedrock living in deep faults in warm anoxic sediments reach in $\mathrm{CO}_{2}$ and hydrogen and sporadically flushing out to the veins into the lake due to a seismotectonic activity.

Cell concentrations in strictly decontaminated ice samples measured by flow cytometry did not exceed 10 cells/ml in both type 1 and 2 accretion ice. All these results point out the very low microbial biomass in the Vostok accretion ice samples, biomass levels two-three orders of magnitude lower than previously published, and leave the question of whether life exists in subglacial Lake Vostok open. Thus, the lake may be viewed as the only extremely clean giant aquatic system on the Earth providing a unique test area for searching for life on icy planets and moons.

\subsection{The enigma of the nitrate deposits in the} Atacama Desert, Chile

${ }^{*}$ Rocco L. Mancinelli ${ }^{1}$, Kimberley Warren-Rhodes ${ }^{1,2}$, Amos Banin $^{1}$, Ragnhild Landheim

${ }^{1}$ Carl Sagan Center for the Study of Life in the Universe, SETI Institute, Mountain View, Moffett Field, CA, USA

${ }^{2} N A S A$ Ames Research Center

Nitrogen is an essential element for life. Understanding the planetary nitrogen cycle is critical to understanding the origin and evolution of life. Of particular significance to prebiotic and biotic chemistry is 'fixed $\mathrm{N}^{\prime}\left(\mathrm{NH}_{3}, \mathrm{NH}_{4^{+}}, \mathrm{NO}_{x}\right.$, organic-N). Without fixed nitrogen life would not have originated on earth and would most likely not occur on any other planet.

The Atacama Desert in Chile is an enigma in that it contains vast nitrate deposits. Elsewhere on earth, nitrate is either denitrified through the activity of microorganisms, or is dissolved and leached from the system. The low water supply (1-2 $\mathrm{mm}$ rainfall $\left.\mathrm{yr}^{-1}\right)$ in the Atacama limits biological activity, including denitrification, and leaching of salts from the soil, and is hypothesized as the primary reason for the observed stability of nitrate. In some parts of the Atacama, such as Yungay $\left(24^{\circ} \mathrm{S}, 69^{\circ} \mathrm{W}\right)$, the soil parent material is volcanic in origin. As a result, these soils contain high concentrations of oxidized transition metal minerals, particularly chromium and manganese, as well as perchlorate. The presence of these oxidized metals stabilizes the soil redox potential at high values, which may inhibit denitrification and also contribute to nitrate stability.

Denitrifiers in most soil environments are ubiquitous and should be in the atmosphere and falling to the desert surface. The results of experiments to isolate and culture denitrifying organisms from Yungay soils were negative. However, fragments of the nir-s gene could be amplified from the soil at a low level, suggesting that denitrifiers occur in the soil. It has been shown that very low concentrations of organic- $\mathrm{C}$ and organic- $\mathrm{N}$ exist in the surface soil, i.e. stable soil organic matter, which indicates very limited biological soil activity.

Rates of denitrification were determined using acetylene to block the reduction of $\mathrm{N}_{2} \mathrm{O}$ to $\mathrm{N}_{2}$. The soil samples were placed into vials fitted with syringe septa, and incubated for at least 10 hours, dry, wet, anaerobic, and aerobic. Controls were run with no acetylene. Periodically, gas samples were collected and analysed via gas chromatography. Rates of nitrogen fixation were determined using the acetylene reduction method. No nitrogen fixation was detected, which is not unexpected due to the abundance of $\mathrm{NO}_{3}$. Denitrification tests were negative, including those from the wet test, suggesting that low water activity alone cannot account for the lack of denitrification in this system. It is known that $\mathrm{O}_{2}$ inhibits denitrification and raises the cell's redox level. Following from this we hypothesize that the high soil redox potential is also responsible for the lack of denitrification. To date, the Yungay region represents the only place in the world where anaerobic denitrification cannot be detected in the presence of nitrate.

\subsection{UV-induced DNA damage in Deinococcus radiodurans: photoproducts and gene expression} *P. Rettberg ${ }^{1}$, U. Pogoda de La Vega ${ }^{1}, T$. Douki ${ }^{2}, J$. Cadet ${ }^{2}$, G. Reitz ${ }^{1}$ ${ }^{1}$ DLR, Institute of Aerospace Medicine, Radiation Biology Division, Köln, Germany

'Laboratoire 'Lésions des Acides Nucléiques', CEA-Grenoble, France Deinococcus radiodurans is a polyextremophilic non-spore forming bacterium. Despite multiple studies aimed at assessing the mechanisms that underlie its unique ionizing radiation resistance only the typical prokaryotic repair pathways like nucleotide excision (NER) and homologous recombination (HR) repair, amongst others, were identified. Our research focuses on the repair pathways of UV-induced DNA damage, because the energy-rich short-wavelength UV 
radiation of the early Earth has been a major driving force in evolution. We investigated the repair-defective mutant strains UVs78 (uvrA1 uvsE, UV damage endonuclease-deficient) and 1R1A (recAdeficient) due to the loss of their UV-related repair and stress response proteins. This was achieved by profiling their transcriptional response through the application of the DNA microarray technology. We first monitored the post-irradiation recovery profile of the UVC $(254 \mathrm{~nm})$ exposed mutant strains and the wild-type strain R1. The UVC-induced photoproducts were measured after different post-irradiation incubation times using high performance liquid chromatography (HPLC) coupled with tandem mass spectrometry. Astonishingly, a recovery time of $0.5 \mathrm{~h}$ was sufficient to give the first clues to the UV-repair process flow: The UV damage endonucleasedependent excision repair (ER) efficiently removes both cyclobutane pyrimidine dimers (CPD) and 6-4 bipyrimidine adducts. HR assists removal of CPD from UV-irradiated DNA, but plays a minor role in the repair of 6-4 adducts. Though the ER is not present in the ER-deficient mutant UVs78, up to $10 \%$ of both bipyrimidine photoproduct types have been equally repaired. Preliminary data analysis of the transcription profile of the D. radiodurans strains differing in their repair capacity, revealed 41 genes that are either up- or down-regulated in all three strains. Of these 41 genes, 18 were identified to belong to the top 100 induced and repressed genes in either strain (3187 open reading frames per microarray of D. radiodurans). Among these 18 identified genes, eight are hypothetical proteins and two are putative transposases, and these eight unknown genes have no gene ontology-conform function attributed so far.
7.4 Growth of microorganisms at Martian subsurface conditions: laboratory modelling

*A. K. Pavlov ${ }^{1,2}$, V. N. Shelegedin ${ }^{3}$, M. A. Vdovina ${ }^{1}$, A. V. Tretyakov ${ }^{1}$

${ }^{1}$ Ioffe Physico-Technical Institute RAS, St. Petersburg, Russia

${ }^{2}$ Russian Astrobiology Center, St. Petersburg, Russia

${ }^{3}$ St. Petersburg Polytechnical State University, St. Petersburg, Russia e-mail: anatoli.pavlov@mail.ioffe.ru

Modern environmental conditions on Mars prohibit the existence of liquid water in the surface layer of Martian soil because of extremely low atmospheric pressure. But according to observational data large amounts of water ice is present in subsurface layers of Mars. In this case ice, is able to undergo intensive sublimation if the surface is heated enough by sunlight. According to TES temperature data, some areas of the Martian surface can be heated up to $300 \mathrm{~K}$ during daytime. Under such conditions vapours diffuse through the porous surface layer. As a result the 'wet layer' appears under the sand's surface. In our experiment we used a special vacuum chamber for the modelling process of ice sublimation and vapour diffusion while heating. In order to model it we used a water ice sample covered by several centimetres of sand with a weight fraction of organic matter (glucose) $\sim 10-5-10-4$. Ice sublimation was driven by radiation heating of the sand's surface.

We studied the possibility of growth of microorganisms in the 'wet layer' under three different temperatures: $280 \mathrm{~K}, 300 \mathrm{~K}$ and $200 \mathrm{~K}$. Bacteria Vibrio sp. X were added to the sand. We carried out several three-day experimental runs of the intensive sublimation of ice. As a result, we discovered an increase of bacterial population in the 'wet layer' after each run at $280 \mathrm{~K}$ and $300 \mathrm{~K}$. These results confirm that shallow ground ice and a few hours of heating per day could provide sufficient conditions for growth of the bacterial population under the Martian surface. Environments of some areas of modern Mars are close to that which we have modelled in our experiments.

\section{Lithopanspermia}

\section{I Shock experiments in support of the} Lithopanspermia theory: the influence of host rock composition, temperature and shock pressure on the survival rate of endolithic and epilithic microorganisms

${ }^{*}$ C. Meyer ${ }^{1}$, D. Stöffler ${ }^{1}$, M. Misgaiski ${ }^{1}$, J. Fritz ${ }^{1}$, R. Moeller ${ }^{2}$ E. Rabbow ${ }^{2}$, G. Horneck ${ }^{2}$, J.-P. De Vera ${ }^{3}$, C. Cockell, U. Hornemann ${ }^{5}$ ${ }^{1}$ Humboldt University Berlin, Museum of Natural History, Berlin, Germany

e-mail:cornelia.meyer@museum.hu-berlin.de

${ }^{2}$ German Aerospace Center, Cologne, Germany

${ }^{3}$ Heinrich Heine University, Institute of Botany, Düsseldorf, Germany ${ }^{4}$ Open University, Milton Keynes, UK

${ }^{5}$ Fraunhofer Institute of High Speed Dynamics, Ernst Mach Institute, Efringen-Kirchen, Germany

${ }^{6}$ Institute of Mineralogy, University of Heidelberg, Germany

Introduction: In view of the geological and climatological development of planet Mars, the origin and evolution of life in the first 1.5 billion years of Martian history appear possible. There is also convincing evidence that a significant amount of surface material was ejected from Mars by impact processes and a substantial portion of it transferred to Earth as meteorites. The Martian meteorites collected so far indicate an exposure to shock waves in the pressure range of 5 to $55 \mathrm{GPa}$ (Fritz et al. 2005). As terrestrial rocks are frequently inhabited by microbial communities, rocks ejected from a planet by impact processes may carry endolithic microorganisms, if microbial life existed/exists on this planet.

Experiment: We performed shock recovery experiments with an explosive set-up in which three types of microorganisms inside various types of host rocks were exposed to strong shock waves: the endospore Bacillus subtilis, the lichen Xanthoria elegans, and the cyanobacterium Chroococcidiopsis sp. 029. In these experiments, three fundamental parameters were systematically varied: (1) the shock pressure, (2) the type of host rock and (3) the pre-shock ambient temperature. The applied pressures were in the range from 5 to $50 \mathrm{GPa}$. The preshock temperatures were set at 293,233, and $193 \mathrm{~K}$ in order to simulate the Martian temperature environment (147 to $290 \mathrm{~K}$ ). The host rocks included non-porous igneous rocks (gabbro and dunite as analogues for the Martian shergottites and chassignites, respectively), porous sandstone, rock salt (halite), and artificial Martian regolith (MRS07) as porous analogues for dry and water saturated Martian regolith.

Results: The experiments with gabbro at an initial temperature of $293 \mathrm{~K}$ show an exponential decrease of the survival rate of the microorganisms with increasing shock pressure up to about $40 \mathrm{GPa}$ where B. subtilis and X. elegans still survived. B. subtilis spores shocked at preshock temperatures of $233 \mathrm{~K}$ or $193 \mathrm{~K}$ survived significantly better than their counterparts shocked at a pre-shock temperature of $293 \mathrm{~K}$. Porosity in host rocks has no influence on the survival rate of $B$. subtlis spores but a negative influence on the survival rate of the vegetative cells of Chroococcidiopsis sp. 029 and X. elegans. The experiments with mafic to ultramafic host rocks (e.g. dunite), which are heated by shock to lower temperatures compared to feldspar-richer rocks (e.g. gabbro) showed higher survival for all tested organisms.

Conclusion: Our data show that the transfer of viable endolithic and epilithic microbes from Mars to Earth is favoured by the low surface and subsurface temperatures of Mars. The experimentally determined survival rates are reasonably high for the Martian temperature conditions and for most of the pressure range observed in Martian meteorites. Survival rates are higher in ultramafic host rocks (corresponding to chassignites, nakhlites) than in basaltic rocks (e.g., shergottites). Therefore, Martian meteorites of the type 'nakhlites' and 'chassignites' are the most favourable carriers for the transfer of living microorganisms from Mars to Earth. Porosity and water saturation in host rocks have a negative influence on the survival rates of microbes. 


\section{Education and public outreach}

\section{I The science and culture of astrobiology in education and outreach \\ * Martin Griffiths and Mark Brake \\ University of Glamorgan, $U K$}

To achieve an effective and inspiring science communication of astrobiology, an educational programme detailing an evolution of both the science and culture of the discipline has been developed. This paper details our cross-curricular approach, which has led to a unique and extensive European Social Funded outreach programme on the possibility of alien life, and the development of a full undergraduate BSc curriculum in astrobiology. The paper details the way in which the science and culture of astrobiology are used in educational programmes at the Centre for Astronomy and Science Education at the University of Glamorgan, Wales, UK. The paper describes how students are encouraged to engage in best practice in the science communication of astrobiology, in order to critically and imaginatively communicate the nature and evolution of the extraterrestrial debate. The programme hopes to illustrate how cultural forms such as science communication and fiction are often unconscious and therefore particularly valuable reflections of the assumptions and attitudes held by society. By virtue of its ability to project and dramatize, science fiction has been a particularly effective, and perhaps for many readers the only, means for generating concern and thought about the social, philosophical and moral consequences of the search for alien life and intelligence.

\subsection{ABC-Net, a European astrobiology lecture course network \\ ${ }^{*}$ Gerda Horneck ${ }^{1}$ and the Abc-Net Team ${ }^{2}$ \\ ${ }^{1}$ German Aerospace Center DLR, Institute of Aerospace Medicine}

${ }^{2} A B C$-Net Team:

Jorge Vago, Pietro Baglioni, Massimo Sabbatini (ESA-ESTEC)

Harry Lehto, Kirsi Lehto (University of Turku)

Francois Raulin, Herve Cottin, Gabriel Tobie (University of Paris 12 \&

Paris 7, University of Nantes)

Stefanos Fasoulas, Helga Stan-Lotter, Heike Rauer, Petra Rettberg (TU

of Dresden, of Salzburg, and of Berlin and DLR)

Fred Goesmann, Dieter Schmitt (MPI Solar System Research,

Universities of Braunschweig and Göttingen)

Raffaele Saladino, A. Rotundi (University of Tuscia, Viterbo, and of Napoli)

Ewa Szuszkiewicz, Franco Ferrari (University of Szczecin)

Maria E. Webb (New University of Lisboa)

Natalia Gontareva (University of St. Petersburg)

Charles Cockell, Monica Grady (Open University, Milton Keynes, UK)

Astrobiology includes the study of the origin, evolution and distribution of life in the Universe. In this endeavour, scientists from a wide variety of disciplines are involved, such as astronomy, planetary research, organic chemistry, palaeontology and the various sub disciplines of biology including microbial ecology and molecular biology. Space technology plays an important part by offering the opportunity for exploring our Solar System, for collecting extraterrestrial samples, and for utilising the peculiar environment of space as a tool. However, because of the multidisciplinary character of astrobiology the full expertise in astrobiology is not always available at a single university thereby rendering it difficult to organize a comprehensive course in astrobiology. The European Space Agency has provided the infrastructure, tools and staff for setting up each lecture as a live television production, broadcasting it in real-time to all other participating university sit technology required for astrobiology missions. They were part of the universities curricula with the possibility of getting European credit points for the students. The lectures were recorded and are available at the ESA web site: http://www.spaceflight.esa.int/exobio under 'Teaching'. They are also published in 'Complete Course in Astrobiology, Horneck G. and Rettberg P. (eds.) Wiley-VCH, Weinheim, Germany, 2007'. The ABC-net will be continued in 2007/ 2008 with a broader participation of European universities.

\section{Young Astrobiologist Session}

\section{Y.I The origin of introns and mRNA}

* Marc P. Hoeppner ${ }^{1}$, Daniel C. Jeffares ${ }^{2}$, Anthony M. Poole ${ }^{1}$

${ }^{1}$ Department of Molecular Biology and Functional Genomics, Stockholm

University, 10691 Stockholm, Sweden

${ }^{2}$ Wellcome Trust Sanger Institute, Wellcome Trust Genome Campus,

Hinxton CB10 1SA, UK

The advent of protein synthesis in an RNA-rich world is considered one of the key events in the transition from RNA- to modern, DNA-based life on Earth. In modern cells, DNA stores the information necessary for protein synthesis, but in return is dependent upon proteins for its replication. It is therefore difficult to imagine how such a relationship originally could have come about. While there has been extensive study devoted to the origin and evolution of the protein synthetic machinery - the ribosome - and to the genetic code, less effort has been devoted to the question of the origin of genes that code for proteins. How might such information arise in a genome, and how could it be expressed? In other words, what is the evolutionary origin of messenger RNA (mRNA)?

We have previously published a hypothesis - introns first (Poole et al. 1998) - which aims to address this question. Briefly, we have argued that some modern introns carry early RNA genes, and that their status as the spacers between protein-encoding exons is a derived trait. Support for this idea comes from the finding that small nucleolar RNAs (snoRNAs) are still transcribed from modern introns. These are found in archaea and eukaryotes, and are involved in the processing and modification of the ribosomal RNAs integral to the ribosome.

Our model argues that there was already a gene expression system in the RNA world, where cleavage was required to produce functional RNAs from larger transcripts. If there were gaps between functional sequences, these would be expressed as a side effect of snoRNA production, and we argue that RNA splicing would be a chemical side effect of this production. Exons in protein-coding genes would therefore have arisen from short stretches of nucleotides between RNA genes, and have been spliced together and expressed as a side-effect of RNA gene expression.

This hypothesis continues to attract interest (Rodriguez-Trelles et al. 2006) in that it solves several outstanding issues in RNA world theory, but has yet to be tested. The presence of snoRNAs in the Last Universal Common Ancestor (LUCA) of the three domains of life would support 
our view of early RNA genes as ancestors of modern introns and strengthen the notion of mRNAs originating as a by-product of early RNA-gene transcription. We will describe an evolutionary bioinformatic analysis of intron-encoded small nucleolar RNAs (snoRNAs) that aims to infer if any of these RNAs can be dated back to the LUCA. Poole, A. M., Jeffares, D. C. \& Penny, D. (1998). The path from the RNA world. J. Mol. Evol. 46, 1-18.

Rodriguez-Trelles, F., Tarrio, R. \& Ayala, F. J. (2006). Origins and evolution of spliceosomal introns. Ann. Rev. Genet. 40, 47-76.

\section{Y.2 The importance of astrobiology for the young generations \\ José Ruiz de La Herrán \\ Universidad Nacional Autónoma de México (UNAM)}

The UNAM is the most important university in Spanish speaking countries, with the main campus south of México City and many campuses in other states. The Institute of astronomy in UNAM operates the National Astronomical Observatory in San Pedro Mártir, Baja California, México.

I am Technical Advisor and founder of the Museo de las Ciencias and Museo de la Luz, both part of the 'Dirección General de Divulgación de las Ciencias' (DGDC), a UNAM institution.

As a separate institution a few years ago, a small group of doctors and professors from UNAM and other universities recently founded in México the 'Sociedad Mexicana de Astrobiología' (SOMA), with Dr. Ramiro Iglesias as its president; the current president is Dr. Rafael Navarro, a well known scientist in this field.

As a member of UNAM and SOMA my interest is in divulgating to the general public in the museums and specially to the young students at schools (from 12 to 22 years old) the great diversity of subjects that astrobiology covers, which have important connections with the many problems of all kinds that we on Earth have been accumulating since the Industrial Revolution.

Letting the young generation know the conditions that a planet has to have in order for life to exist will help them to better understand the way we must in the immediate future behave to maintain good living conditions on our small Earth. But astrobiology is much more than that. As it gives a broader scope of our Universe, it help us humans to look at the 'Big Picture' and to better understand our mission that connects science, industry, social behavior, health and nature to direct our actions for obtaining a better way of living in this globalized world.

Astrobiology, apart from its scientific activities, works in the mind of humans in many other fields of thinking and helps us to comprehend more profoundly our responsibilities in the present and the future.

\section{Y.3 Irradiation of a homogeneous mixture of ammonia and carbon dioxide $\left(\mathrm{NH}_{3}, \mathrm{CO}_{2}\right)$ at low temperatures}

*Sohan Jheeta ${ }^{1}$, Anne Lafosse ${ }^{2}$, Bhalamurugan Sivaraman ${ }^{1}$, Slywia Ptasinska ${ }^{1}$, Nigel Mason ${ }^{1}$

${ }^{1}$ The Open University, Depart. of Physics \& Astronomy, Walton Hall, Milton Keynes, UK

${ }^{2}$ Laboratorie des Collisions Atomiques et Moleculaires, Paris, France Carbon dioxide and ammonia are two of the most abundant ices to be found in the Solar System. Carbon dioxide ice is prevalent on Mars and Jupiter, whilst ammonia ice has recently been detected in the upper atmosphere of Saturn and is a strong surface signature of Pluto, with ammonia hydrate having been detected on the surface of several Kuiper belt objects (e.g. Quaoar). A comprehensive study of the processing of such ices under planetary conditions is important to ascertain if life existed elsewhere other than on Earth.

In this experiment we report the results of electron irradiation of an omogenous gaseous mixture of $\mathrm{NH}_{4}$ and $\mathrm{CO}_{2}$ deposited on a zinc selenide $(\mathrm{ZnSe})$ substrate at a temperature of $\sim 30 \mathrm{~K}$. The electron energy used to irradiate the resulting ice formation was in the region of
$1 \mathrm{keV}$ and electron currents of $\sim 10 \mu \mathrm{A}$ were used. The results were analysed by infrared spectroscopy and are outlined below:

(a) Two new IR peaks were observed. The first peak was at $2140 \mathrm{~cm}^{-1}$ and is associated with a stretching vibration of the CO molecule. The second was at $\sim 2167 \mathrm{~cm}^{-1}$ and this was attributed to $v_{\mathrm{as}}(\mathrm{N}=\mathrm{C}=0)$ of $\mathrm{OCN}$-ions by Brucato et al. (2006) and Raunier et al. (2003), and to a nitrile compound containing some $\mathrm{O}$ that is bound to the organic residue $v(\mathrm{R}-\mathrm{O}-\mathrm{C} \equiv \mathrm{N})$ by Palumbo et al. (2000). This suggests that electron induced chemistry within the ice is liberating a rich range of reactive species;

(b) When the temperature of the irradiated sample was slowly raised (220-270 K), ammonium carbamate was formed (Frasco 1964).

These preliminary investigations lend themselves for future experimentation in that the effect of lower electron energies $(20-500 \mathrm{eV})$ levels should be explored. Such energy levels are feature of various Solar System bodies mentioned above.

Brucato, J. R., Baratta, G. A. \& Strazzulla, G. (2006). An infrared study of pure and ion inrradiated frozen formamide. Astron. Astrophys. 455, 395 .

Raunier, S., Chiavassa, T., Marinelli, F., Allouche, A. \& Aycard, J.-A. (2003). An infrared and theoretical study about $<<\mathrm{XCN}\rangle>$ band formation: reactivity of $\mathrm{HCNO}$ with $\mathrm{NH}_{3}$ astrophysical ice laboratory analogues and spontaneous production of OCN. J. Phys. Chem. A 107, 9335.

Palumbo, M., Strazzula, Y., Pendleton, Y. \& Tielens, A. (2000). R$\mathrm{O}-\mathrm{C}=\mathrm{N}$ species produced by ion irradiation of ice mixture: comparison with astronomical observations. Astrophys. J. 534, 534.

Frasco, J. (1964). Infrared spectra of ammonium carbamate and deuteroammonium carbamate. Chem. Phys. 41, 2134.

\section{Y.4 Dissociative recombination of nitrile} ions - important processes in Titan's atmosphere ${ }^{*}$ Erik Vigren ${ }^{1,2}$, Magdalena Kaminska ${ }^{1,3}$, Vitali Zhaunerchyk ${ }^{1}$,

Mathias Hamberg ${ }^{1}$, Mathias Danielsson ${ }^{1}$, Richard D. Thomas,

Jacek Semaniak $^{2}$, Patrik Andersson ${ }^{4}$, Mats Larsson ${ }^{1}$, Wolf D. Geppert ${ }^{1}$

${ }^{1}$ Department of Physics, AlbaNova, Stockholm University, SE-10691

Stockholm, Sweden

${ }^{2}$ Astrobiology Graduate School, Stockholm University, Stockholm,

Sweden,

www.astrobiology.physto.se

${ }^{3}$ Institute of Physics, Swietokrzyska Academy, Swietokrzyska 15, PL25406 Kielce, Poland

${ }^{4}$ Department of Chemistry, Atmospheric Science, Göteborg University, SE-41296 Göteborg, Sweden

During a Titan flyby on April 16 2005, the Ion Neutral Mass Spectrometer on board the Cassini spacecraft recorded surprisingly strong mass over charge signals at (for example) $m / z=42,54$ and 56 . These signals are believed to come from protonated acetonitrile $\left(\mathrm{CH}_{3} \mathrm{CNH}+\right)$, protonated acrylonitrile $\left(\mathrm{C}_{2} \mathrm{H}_{3} \mathrm{CNH}+\right)$ and protonated propionitrile $\left(\mathrm{C}_{2} \mathrm{H}_{5} \mathrm{CNH}+\right)$, respectively (Vuitton et al. 2006). Acetonitrile $\left(\mathrm{CH}_{3} \mathrm{CN}\right)$ was detected in the atmosphere of Titan in 2002 and its abundance was much higher than what was expected from earlier model calculations (Marten et al. 2002). These discoveries indicate that the nitrile chemistry of Titan's atmosphere is much more important than previously believed.

To improve the models of Titan's atmosphere the formation and destruction routes of nitriles and nitrile ions need to be investigated. Dissociative recombination (DR) (the reaction between an electron and a molecular ion, leading to dissociation of the highly excited intermediate into neutral products) is vital for the destruction of molecular ions in planetary ionospheres. Not only is DR a crucial pathway for ion destruction, it can also be a final step in production of neutral molecules. For example, acetonitrile can become protonated by hydrocarbon ions present in Titan's ionosphere. The produced ions can undergo DR leading to acetonitrile and a free hydrogen atom (in which case acetonitrile has been recycled) or other fragments, which results in net destruction of the nitrile. In DR reactions of polyatomic molecular ions often many pathways are exoergic. Therefore, exact 
knowledge of rate coefficients and branching ratios of the DR of ions abundant in Titan's ionosphere is vital for the quality of model predictions.

Motivated by the Cassini results we have investigated the DR of protonated acetonitrile and protonated acrylonitrile at the heavy ion storage ring CRYRING in Stockholm. Branching fractions, cross sections and thermal rate coefficients have been deduced for the DR of both ions. The methods and results of these experimental studies will be presented alongside implications for the atmosphere of Titan.

Marten, A. et al. (2002). Icarus 158, 532.

Vuitton, V. et al. (2006). Astrophys. J. Lett. 647, 175.

\section{Y.5 Compound model to explain water origins for \\ Earth-like planets \\ * Karla de Souza Torres ${ }^{1}$, Othon Cabo Winter ${ }^{2}$ \\ ${ }^{1}$ Instituto Nacional de Pesquisas Espaciais (INPE), Brazil \\ ${ }^{2}$ Faculdade de Engenharia De Guaratinguetá (Feg/Unesp), Brazil}

In its gaseous and solid forms, water is present in the most distant galaxies, among the stars, in the Sun, in its planets and their satellites and ring systems, and in comets. In its liquid form, it has played an essential part in the appearance, development and maintenance of terrestrial life. The origin of water on Earth remains one of the most important subjects of debate and controversy in Solar System formation science. Possible sources of water can be divided into endogenous and exogenous. The most accepted endogenous source is the absorption of water from gas onto grains in the accretion disk. The most accepted exogenous source is that the bulk of the Earth's water may have come from the asteroid belt in the form of planetary embryos with up to $10 \%$ of water by mass. However, no sole source of water is enough to explain Earth's water as a whole. We have composed a compound model incorporating both the principal endogenous and exogenous theories and we use dynamical simulations of planetary formation and water delivery to investigate the implications of this. We analyse our results using chemical constraints like $\mathrm{D} / \mathrm{H}$ ratio as the discriminator. The goal is to understand how terrestrial planets got their water in the Solar System and expand it to extrasolar systems. From the results we can conclude that the compound model incorporating both main theories better explains the D/H ratio of Earth's water. We are now performing an analysis also considering the impact of a cometary component in the model. Future work will apply these results to other planetary system data.

\section{Y.6 Structure and evolution of RNA polymerases Liisa Gunnelius University of Turku, Turku, Finland}

The purpose of this presentation is to give a general view of shared and lineage-specific features of different RNA polymerases, focusing on cyanobacterial RNA polymerase (RNAP) and its close relative, the plastid-encoded polymerase (PEP).

We start with bacteria, since they have only one RNA polymerase. The bacterial RNAP core enzyme consists of beta and beta-prime subunits, which form a channel for the DNA-to-RNA-polymerase reaction; two identical alpha subunits, which bind to an upstream promoter element for enhanced reaction rate; and an omega subunit, which is a chaperone protein that helps the beta-prime subunit in folding and assembly to core. For initiation and promoter recognition, bacteria rely on the sigma factor subunit. Bacteria usually have more than one kind of sigma factor. The most interesting group is the sigma70, which includes three subgroups: group 1 are the so-called primary sigmas, which are responsible for transcription of housekeeping genes and therefore usually cannot be deleted from the genome, group 2 are the primary-like or alternative sigmas and group 3 are more distantly related. E. coli has only one group 2 sigma factor, but cyanobacteria typically have a few. Alternative sigma factors are functional in various stresses and developmental stages. Because the DNA-binding amino acids are very conserved across species and even between group 1 and 2 sigmas, promoter specificity probably lies in the variable areas. A strong candidate might be the large non-conserved domain between conserved regions 1 and 2 , which in the three-dimensional structure comes near the DNA at -10 promoter element.

In plants, the plastome contains the core subunits (except omega, which has presumably been lost at some point of evolution) whereas the sigma factor genes have been transferred to the nucleus. Arabidopsis, for example, has six genes encoding PEP-associated sigma factors. Some of these have evolved after the endosymbiotic event. However, none of them seems to be absolutely necessary to the plant, indicating that there is no primary sigma factor for the PEP. This is understandable because even a nucleus-encoded polymerase (NEP) exists in plastids. The NEP is similar to the mitochondrial polymerase (also nucleus-encoded), a T7 phage-type single polypeptide polymerase that is solely responsible for all transcription in mitochondria. In fact, there are indications that the NEP has evolved from the mitochondrial enzyme. Eukaryotes in general have several RNA polymerases, both for the transcription of DNA and replication of endogenous or other RNA, which plays an important role in development and defence against viruses. These RNA-dependent RNA polymerases also share subunit homology with the bacterial RNAP. The enzyme that is responsible for transcription of protein-coding DNA is called RNA Polymerase II and the polymerization core has high sequence similarity with the bacterial core, but there are additional subunits. Eukaryotes do not have sigma factors either but need several other transcription factors for initiation. Archaea also have several RNA polymerases and initiation factors, and the whole system resembles more the eukaryan system.

\section{Y.7 Analysis of the critical moments of the evolution of biosphere macroparameters for revealing catastrophes \\ *V. A. Ozheredov, N. G. Khorseva}

Space Research Institute RAS, Profsoyznaya 84/32, 117997 Moscow, Russia

e-mail: ojymail@mail.ru

Daily data over 28 years characterizing the population parameters of mortality and 2 years of daily blood pressure measurements have been analysed for revealing so-called 'phase-flow tubes', invented to be harbingers of catastrophes in the biosphere. In order to arrange variations of bioparameters by relaxation, time spectral analysis was substituted by GAP-factor analysis allowing rearrangement of the pool of signal components into trends and oscillations. Lack of oscillations means the onset of catastrophes. The moment of the phaseflow tube's appearance was characterized by a prominent shift of the GAP-spectrum obtained from decomposition by eigenfunctions of the GAP-operator to the components of a smooth trend. The advantages of such an approach in comparison with traditional spectral analysis are:

(1) We can now operate in terms of 'trend' and 'oscillations' in the case of lack of information about the model of randomization of the time series while usage of the 'noise' term is absolutely incorrect due to the absence of a valid model of the considered system.

(2) We had the opportunity to describe the relation between mutual trends and oscillations of the arbitrary number of variables by means of a single spectrum.

(3) We need significantly less spectral components in order to describe the behavior of time series fairly well (the qualitative estimator is a determination coefficient).

It was clearly shown that phase-flow tubes are present in explored mortality time series and the appearance of them is correlated with averaged Kp-index. We have also shown that the GAP-spectrum of blood pressure has prominent seasonal variation. 


\section{Y.8 Searching for habitable-zone planets with SuperWASP \\ *D. R. Anderson ${ }^{1}$ and The Superwasp Consortium ${ }^{2}$ \\ ${ }^{1}$ Astrophysics Group, Keele University, Keele, UK \\ ${ }^{2}$ The SuperWASP consortium comprises Cambridge University, the Instituto de Astrofisica de Canarias, the Isaac Newton Group of telescopes, Keele University, Leicester University, the Open University, Queen's University Belfast and St. Andrew's University}

A planetary transit occurs when a planet passes in front of its parent star along the line of site, causing a small dip in the measured brightness of the star. Ground-based transit surveys are focusing on the search for Hot-Jupiters, and certainly the surveys are most sensitive to these objects. The discovery of Hot-Jupiters provides us with statistics on planetary systems and places constraints on theories of planet formation. This will enable us to understand what type of planetary systems exist and at what frequency, which has implications for the search for life. However, a more directly applicable quest is the search for habitable planets. I propose to perform such a search around red dwarf stars.

Red dwarfs are small, cool stars with masses less than half of that of the Sun. They are the most common type of star in the Galaxy, making up around $70 \%$. The small size of red dwarfs mean that a planetary transit of the star will be of greater depth and therefore easier to detect. As red dwarfs are cool, their habitable zones lie closer to the star than is the case in our own Solar System. This facilitates detection in two ways: the probability of a transiting alignment is higher; a smaller baseline of observations is required to see one or more transits.

The detection of Jovian-size planets around red dwarfs opens up the possibility of detecting terrestrial bodies in the same system. A terrestrial planet can influence the orbit of the Jovian-size planet sufficiently that the transit period of the massive planet varies by a detectable amount (Transit Timing Variations). The effect will be greatest for planets in mean motion resonances and would be detectable for Trojans (bodies in the same orbit as the massive planet).

A Jovian-size planet in the habitable zone of a red dwarf may have habitable terrestrial moons. Precise photometry could detect the transit signature of the moon in the transit profile of the massive planet.
SuperWASP (Wide Angle Search for Planets) is a UK-led, groundbased transit survey responsible for the discovery of two Hot-Jupiters to date. SuperWASP is being used to search for Jovian-size planets in the habitable zone of red dwarfs. If any are found, follow-up observations on other instruments will be used to search for terrestrial, potentially habitable, planets and moons.

\section{Y.9 How to close the door leaving it open? - On the origin of membrane transport system \\ Katarzyna Adamala}

Center for Computational and Integrative Biology, Massachusetts General Hospital, Boston, MA, USA

The evolution of cellular life, with cells as individual specimens, become possible only when various cycles of primitive metabolic reactions were separated from the environment and from each other. Thus, to the origin of life, encapsulation of protometabolic reactions inside vesicle compartments was necessary.

However, encapsulation of primitive metabolism would be no advantage at all without immediate origin of selective membrane transport system. Encapsulated metabolism must have been able to exchange matter and energy with the environment, uptake nutrients, remove waste and collect information about environmental changes. The cross-membrane gradients of concentration of various compounds must have been kept from the very beginning of life's encapsulated history. To maintain these gradients, a trans-membrane system of channels and pores must have been able to provide selective permeability of inorganic ions and small organic molecules. There are three main groups of compound potentially able to provide transport across bilayer membrane. Peptides, nucleic acids and small organic compounds can all serve as efficient channels and carriers. However, there are various problems of availability of these compounds and conditions required to channel formation. During this talk, the main hypotheses explaining the primitive transport system will be presented, along with some experimental data supporting some of these hypotheses.

\section{POSTERS}

\section{PI. Life}

\begin{abstract}
PI.I What is life?
Anthony Mellersh and Sohan Jheeta

Department of Physics \& Astronomy, The Open University, Walton Hall, Milton Keynes MK7 6AA, UK

Current definitions of life are unsatisfactory. For instance the NASA definition 'a self-sustaining system capable of Darwinian evolution'; is not really very helpful because life requires input from the environment and 'Darwinian evolution' is itself not well defined. Similarly the M.E.R.R.I.N.G. criteria leave room for doubt. Bacterial spores do not move ( $\mathrm{M}$ criterion) while crystals and fire grow $(\mathrm{G})$. Are post-menopausal women dead because they cannot reproduce (R)? Neither definition is useful in the search for extraterrestrial life. We propose, for discussion, a new definition, which we feel overcomes some of these problems.
\end{abstract}

\section{PI.2 Computer simulated macroevolution: beyond Bak-Sneppen and generalized Lotka-Voltera models Wojciech Borkowski \\ Institute for Social Studies, Warsaw University, Poland}

Christopher G. Langton, the 'godfather' of Artificial Life researches said: 'Biology is the scientific study of life-in principle, anyway.
In practice, biology is the scientific study of life on Earth based on carbon-chain chemistry. There is nothing in its charter that restricts biology to carbon-based life; it is simply that this is the only kind of life that has been available to study.' This problem especially concerns Astrobiology, which should consider other examples for extracting most universal properties of life, but for now cannot, in the absence of any alien life forms available for study.

For such reasons astrobiologists are currently mostly 'astrobio-physico-chemists', but a different way is possible - we can recreate important biological phenomena from scratch using computers or other artificial media. My simulation model presented here is just one, I hope interesting, example: the term 'macroevolution' refers to biological evolution that occurs at or above the level of species. Because it acts on the ecosystems and the whole biosphere level in very long timescales, even on the Earth macroevolution is not available for experimental research - only post-factum observations are possible. So, like in astrophysics, simulation models are very important for connecting known facts and deep understanding of observable processes and patterns, but the current 'dominating', physically originated models: Bak-Sneppen, Generalized Lotka-Voltera Models and Agend Based Predator-Prey Systems, each lack different, but important aspects of biological evolution, so they are not attractive for the real 
biologist. In opposition to them, I tried to make a Cellular Automata like model, with as simple as possible rules, which takes care of a few of the most important aspects: potential infinity of space of ecological niches including places both for specialists and opportunists, random searching by speciation (the emergence of new species), and the limiting role of local energy transfer between interacting individuals. Because of the importance of multispecies co-evolution the model is called COEVO.

A dynamic of this simulation recreates what is known from behaviours of popular models and also many other 'patterns' known from real paleobiology. One can observe the Lotka-Voltera like cycles - locally in short time scale, and Bak-Sneppen like punctuated equilibrium and 'phylogenetic trees'-globally in a large time scale, and also important phenomena such as adaptive radiations, spontaneous increasing of ecosystem complexity, correlation between availability of abiotic resources and ecosystem complexity, and especially chaotic emergence of smaller, larger and mass extinctions for which 'specialization trap' and 'key innovation escape' play a crucial role, but catastrophic changes in environment are not necessary.

\section{P2. Life, climate and the atmosphere}

\section{P2.I The habitability of super-Earths in Gliese 58I \\ *W. von Bloh ${ }^{1}$, C. Bounama ${ }^{1}, M$. Cuntz ${ }^{2}$, S. Franck ${ }^{1}$ \\ ${ }^{1}$ Potsdam Institute for Climate Impact Research, PO Box 601203 , 14412 Potsdam, Germany \\ ${ }^{2}$ Department of Physics, University of Texas at Arlington, Box 19059, Arlington, TX 76019, USA}

The planetary system around the $\mathrm{M}$ star Gliese 581 consists of a hot Neptune (Gl 581b) and two super-Earths (Gl 581c and Gl 581d) with 5.06 and 8.3 Earth masses with a semi-major axis of $0.073 \mathrm{AU}$ and $0.25 \mathrm{AU}$, respectively. Super-Earths are rocky planets from one to ten Earth masses with a chemical and mineral composition similar to Earth. The habitability of this system with respect to the superEarths is investigated following a concept that studies the long-term possibility of photosynthetic biomass production on a dynamically active planet.

The habitable zone is determined by the limits of photosynthetic life on the planetary surface. Therefore, all the pertinent astrophysical, climatological, biogeochemical, and geodynamic processes involved in the generation of photosynthesis-driven life conditions are taken into account. The main point is the persistent balance between carbon dioxide in the atmosphere/ocean system and the metamorphic (plate tectonic) sources. A thermal evolution model for a super-Earth is used to calculate the sources and sinks of atmospheric carbon dioxide. We use scaling laws to obtain total radius, mantle thickness and average density as a function of planetary mass. Models with different ratios of land/ocean coverage are investigated.

We find that the super-Earth Gl 581c is clearly outside the habitable zone, since it is too close to the star. In contrast, Gl 581d is a tidally locked habitable super-Earth near the outer edge of the habitable zone. A planet with eight Earth masses has more volatiles than an Earth size planet to build up a sufficiently dense atmosphere. This prevents the atmosphere from freezing out due to tidal locking. Despite the adverse conditions on this planet, at least some primitive forms of life may be able to exist on its surface. Therefore Gl 581d is an interesting target for the planned TPF/Darwin missions to search for biomarkers in planetary atmospheres.

\section{P2.2 Simulating terrestrial effects of a nearby supernova \\ *Dimitra Atri, Adrian L. Melott, Brian C. Thomas}

Department of Physics and Astronomy, University of Kansas, USA

Several observations suggest frequent supernova events in the Solar neighbourhood in the past couple of million years. There is a direct geochemical evidence of such an event, which occurred about 2.8 million years ago at an estimated distance of about $40 \mathrm{pc}$. We examine the effects of such an event on the Earth's atmosphere and the resulting biological implications. We investigate ozone depletion, the subsequent increase of Solar UVB reaching the Earth's surface, and the resulting biological impact.

\section{P2.3 Conditions for lightning in gas-dusty atmosphere of water-containing bodies of the Solar System Yuriy Serozhkin Institute of Semiconductor Physics, Kyiv, Ukraine e-mail: yuriy.serozhkin@zeos.net} After the Miller and Urey experiments (Miller 1953) electric discharges are consider as one of the energy sources for the synthesis of biochemical compounds both in Earth's atmosphere (Chyba \& Sagan 1991) and in atmospheres of others bodies of the Solar System (Navarro-Gonzalez et al. 2001; Desch et al. 2002). Numerous theoretical and experimental researches of gas-grain chemistry show that the chemical reactions on the gas-ice boundary play a considerable role in changes in the evolution of molecular composition of gas-grain media (Allamandola \& Hudgins 2003; Hugh et al. 2003).

The real role of the electrical discharge in the synthesis of biochemical compounds is not clear for several reasons. The discharges used in experiments on the synthesis of organic substances do not replicate the discharges observable in a nature. What types of discharges occur, and in what conditions, can play a substantial role in the synthesis of biochemical compounds.

As practically all terrestrial lightning is connected with the presence of water, we should be ready to acknowledge that in the waterless atmosphere the minimum probability of lightning exists. In this connection the greatest interest (in the opinion of the author) represents searching for electrical activity or tracks of such activity in the form of chemical species simulated by electrical discharges in atmospheres of water-containing bodies of a Solar System (comets, ice satellites of Jove and Saturn). In the submitted work the conditions for origin of electrical discharges in near-surface atmospheres of these bodies are analysed. From the point of view of the development of electrical discharges, the gas-dusty cometary atmosphere is an extremely interesting object because of the presence of water and ice particles. The atmosphere of comets is a complicated dynamic object, in which there is a directional motion of particles, convection, melting of ice and fracture of large particles.

In at least two cases above, where a surface of ice satellites transiently, there can be an atmosphere with physical performances that gives the hypothetical possibility for accumulation of charges on a dust component and subsequent discharges. The first case is connected to the eruption of a volcano and cryovolcano, and the second with the origin of an atmosphere above places of bombardment of an ice surface by small bodies.

Miller, S. L. (1953). Science 117, 528.

Chyba, C. \& Sagan, C. (1991). Electrical energy sources for organic synthesis on the early Earth. Orig. Life Evol. Biosph. 21, 3-17.

Navarro-Gonzalez, R., Ramirez, S. I., de la Rosa, J. G., Coll, P. \& Raulin, F. (2001). Production of hydrocarbons and nitriles by electrical processes in Titan's atmosphere. Adv. Space Res. 27(2), 271-282. 
Desch, S. J., Borucki, W. J., Russell, C. T. \& Bar-Nun, A. (2002). Progress in planetary lightning. Rep. Prog. Phys. 65, 955-997.

Allamandola, L. J. \& Hudgins, D. M. (2003). From interstellar polycyclic aromatic hydrocarbons and ice to astrobiology. In Proc of the NATO ASI entitled 'Solid State Astrochemistry', ed. Pirronello, V. \& Krelowski, J. Kluwer, Dordrecht.

Hill, H. G. M. \& Nuth, J. A. (2003). the catalytic potential of cosmic dust: implications for prebiotic chemistry in the solar nebula and other protoplanetary systems. Astrobiology 3(2), 291-304.
P2.4 Evolution of Earth-size planetary atmospheres: from planets to exoplanets

*Kaijun Liu, Riku Järvinen, Ilkka Sillanpää, Walter Schmidt, Esa Kallio, Pekka Janhunen

Finnish Meteorological Institute, Helsinki, Finland

We present some preliminary results from our 3D quasi-neutral hybrid (QNH) simulations of planet/exoplanet-Solar/stellar wind interactions. The QNH model has been successfully applied to the study of different planets, such as Mercury, Venus, and Mars. Applying the model to other planets/exoplanets, which have different ages, will help us to understand the evolution of Earth-size planetary atmospheres. This also provides unique new possibilities to study the formation and evolution of planetary objects in our Solar System and beyond.

\section{P3. Mars}

P3.I Identification of $\beta$-carotene in a Martian-analog evaporitic matrix using Raman spectroscopy - a methodical approach

*P. Vitek, K. Osterrothová, J. Jehlicka

Charles University, Institute of Geochemistry, Mineralogy and Mineral Resources, Albertov 6, 12843 Prague 2, Czech Republic

In the search for traces of life on Mars $\beta$-carotene serves as a potential biomarker molecule. Not only is it a UV-protective pigment due to its screening effect, but it also functions as a quenching agent. From terrestrial conditions we know that a variety of extremophilic microorganisms develop this pigment in order to survive harsh conditions resulting from high UV doses. Due to the presence of harmful UV radiation on the Martian surface, microbial life, if present on Mars, should produce these UV-protecting compounds as part of its survival strategy. In cases where external conditions do not allow for surface growth, rocks become the sanctuary for microorganisms that need additional protection. The rocks to which the microorganisms tend to migrate are usually transparent, such as gypsum or halite. The transparency of these rocks allows radiation to reach the microorganisms in the deeper mineral layers, which in turn allow photosynthesis. As a result, these rocks can be considered potential Martian geological habitats.

Raman spectroscopy was tested as a non-destructive method of determining the presence of $\beta$-carotene in experimentally prepared mineral matrices. Samples prepared by mixing $\beta$-carotene with powdered gypsum, halite and epsomite were analysed using two different excitation wavelengths $(514 \mathrm{~nm}$ and $785 \mathrm{~nm})$. Various concentrations of $\beta$-carotene in the matrix were investigated to determine the detection limits of this biomarker. We situated $\beta$-carotene mixed with halite or epsomite first inside the UV-transparent crystals of gypsum $\left(\mathrm{CaSO}_{4}\right.$ $\left.2 \mathrm{H}_{2} \mathrm{O}\right)$ and then in the frame of epsomite $\left(\mathrm{MgSO}_{4} 7 \mathrm{H}_{2} \mathrm{O}\right)$, thereby creating artificial inclusions similar to those that could be present in Martian minerals. All the samples were analysed using two excitation wavelengths. $100 \mathrm{ppm}$ of $\beta$-carotene mixed with halite inside the gypsum crystal was easily detected. If a similar concentration of $\beta$-carotene exists in the Martian samples, then Raman spectroscopy will be able to identify it. These results will aid both in-situ analyses on Mars and sample analyses on Earth. Studies are in progress to develop Raman techniques and to achieve lower detection limits.

\section{P3.2 A low wax crude oil could explain possible liquid hydrocarbon seeps on Mars \\ *M. S. Direito, M. E. Webb \\ Faculty of Sciences and Technology, New University of Lisbon, 2829- \\ 516 Campus Caparica, Portugal \\ e-mail:ew@fct.unl.pt}

We have previously proposed the possible existence of liquid hydrocarbon seepages on Mars. Indeed, we suggested that the dark slope streaks at the Mars equatorial region and some dark features at the Martian South Pole visible on Mars Global Surveyor (MGS) Mars Orbital Camera (MOC) images could be oil or oily-like mixtures of hydrocarbons flowing to the surface of this planet (Direito \& Webb 2007). In fact, we suggested that the dark slope streaks could be liquid hydrocarbon seepages or mixtures of liquid hydrocarbons and water. The observed seeps cannot be just water, since the current Martian conditions don't allow water in liquid state at the planet's surface. Liquid water would freeze due to the low temperatures or would rapidly evaporate due to the current low atmospheric pressure (mean value of 5.6 mbar) (Horneck 2000). Currently, we propose that a naphthenic crude oil type could easily explain how liquid hydrocarbons could flow at the currently low Martian temperatures. Indeed, on Earth naphthenic oils have lower pour points (the lowest temperature oil is observed to flow) due to the absence of waxes (United States Army Corps of Engineers 1999). On our planet, many natural occurring naphthenic crude oils have pour points below $273.15 \mathrm{~K}$.

Direito, M. S. \& Webb, M. E. (2007). Search for oil reservoirs on Mars (European Astrobiology Network Association 2006 Meeting Abstracts). Int. J. Astrobiol. 6(1), 62-63.

Horneck, G. (2000). The microbial world and the case for Mars. Planetary Space Sci. 48, 1053-1063.

United States Army Corps of Engineers (1999). Types of oil. In Lubricating Oils, Lubricant and Hydraulic Fluids, Engineer Manuals, ch. 3. Headquarters USACE Publication Bulletins http://www.usace.army. $\mathrm{mil} /$ publications/eng-manuals/em1110-2-1424/c-3.pdf.

\section{P3.3 Raman LIBS Instrument for ExoMars 2013:} calibration and data refining procedures

N. Tarcea ${ }^{1}$, T. Dörfer ${ }^{1}$, M. Schmitt ${ }^{1}$, M. Hilchenbach ${ }^{3}$, H. Thiele ${ }^{4}$, H. Henkel ${ }^{5}$, I. Rauschenbach ${ }^{6}$, E. K. Jessberger ${ }^{6}, F$. Rull , R. Hochleitner ${ }^{8}$, F. Langenhorst ${ }^{9},{ }^{*} J$. Popp ${ }^{1,2}$

${ }^{1}$ Institute for Physical Chemistry, University of Jena, Jena, Germany ${ }^{2}$ Institute of Photonic Technology (IPHT), Jena, Germany

${ }^{3}$ Max-Plank-Institut für Sonnensystemforschung, Katlenburg-Lindau, Germany

${ }^{4}$ Kayser-Threde GmbH, Munich, Germany

${ }^{5}$ von Hoerner \& Sulger GmbH, Schwetzingen, Germany

${ }^{6}$ Institute für Planetologie, Westfälische Wilhelms Universität, Münster, Germany

${ }^{7}$ Cristalografia y Mineralogía, Universidad de Valladolid

${ }^{8}$ Mineralogische Staatssammlung München, Munich, Germany

${ }^{9}$ Institut für Geowissenschaften, University of Jena, Germany

*e-mail: juergen.popp@uni-jena.de

ExoMars is the first mission of the European Space Exploration Program Aurora. For this mission, planned to fly to Mars on 2013, new scientific instrumentation is considered. One of the new instruments that are being developed as part of the scientific exobiology instrumentation payload is a combined Raman-LIBS device. 
The combined Raman-LIBS instrument aims at detecting key molecular Raman fingerprints and elemental composition (via LIBS) for the assessment of mineral and organic materials. Establishing the biologic origin of the findings is of utmost importance and might be achieved by the synergy of the two methods (Raman and LIBS) when measurements are made on the very same spot. A precise calibration of the instrument under Martian environmental conditions and unambiguous spectral data collected in the laboratory for supporting the analysis of the instrumental data is a must for the success of this undertaking.

If organics are found on Mars, the question of their origin becomes prominent. Their presence may reflect biological processes or they may be the result of meteoritic input and/or abiotic synthesis. Organics that derive from biological processes (metabolism products or dead organisms) differ from the ones created via non-biological processes in several aspects. As observed on Earth, biology is selective and specific in its use of molecules. For example, Earth life uses 20 left-handed amino acids and biologic activity usually leaves characteristic isotopic patterns. Biological activity often also produces large complex molecules in high concentrations, for example the protective pigments of photosynthetic organisms in extreme Antarctic deserts.

The spectral fingerprints (for both Raman and LIBS) of molecules produced by these different processes differ but the differences are usually extremely small and might be undetected. For a given sensitivity and spectral resolution of the instrument the spectral assignment accuracy is highly dependent on the calibration precision. Methods of achieving a good calibration of the instrument are discussed. Also the correct and unambiguous spectral identification have to be assured. This implies reaching back to unambiguous spectral data measured in the laboratory and eventually organized in a Raman-LIBS data repository. The amount of laboratory data needed for a comprehensive repository is huge therefore the contributions from the Raman and LIBS communities have to be brought together. A possible way to achieve a standardization of the laboratory scientific data aimed to be used for the Raman-LIBS experiment on Mars is proposed. Also a Raman-LIBS community platform for standards and data exchanges will be presented with the expectation that the scientific Raman-LIBS community might this way better concentrate the effort towards achieving the main goals of the Raman-LIBS experiment on Mars.

\section{P3.4 The effect of short wavelength UV radiation} on uracil thin layer. An application of the 'Mars lamp' *A. Bérces ${ }^{1}$, G. Kovács ${ }^{1}$, H. Lammer ${ }^{2}$, Ch. Kolb ${ }^{2}$, Gy. Rontó ${ }^{1}$

${ }^{1} M T A-S E$ Research Group for Biophysics of Hungarian Academy of Sciences, Budapest, POB 263, H-1444, Hungary

${ }^{2}$ Space Research Institute, Department for Extraterrestrial Physics, Austrian Academy of Sciences Schmiedlstr. 6, A-8042, Graz, Austria Ultraviolet (UV) radiation can act on putative organic/biological matter at the Martian surface in several ways. Only absorbed, but not transmitted or reflected, radiation energy can be photo-chemically effective. The most important biological UV effects are due to photochemical reactions in nucleic acids, DNA or RNA, which constitute the genetic material of all cellular organisms and viruses. UV radiation can induce wavelengths-specific types of DNA damage. At the same time it can also induce the photo-reversion reaction of a UV induced DNA photoproduct of nucleic acid bases, the pyrimidine dimers. Intense UVB and UVC radiation, experienced on early Earth and present-day Mars, has been revealed to be harmful to all organisms, including extremophile bacteria and spores. Following this, more laboratory simulations are vital in order to investigate and understand UV effects on organic matter in the case of Mars. We have designed a 'Mars lamp' that simulates the anticipated Martian UV surface spectrum between 200 and $400 \mathrm{~nm}$ (UVC-UVA). The Mars-UV simulator represents a device for several different Mars simulation facilities with specific emphasis on Mars research topics.

The dimerization and reversion efficiency of the ultraviolet radiation in the UVB and the UVC range were quantified on polycrystalline uracil thin layers irradiated with quasi-monochromatic radiation using interference filters of a bandwidth of $10 \mathrm{~nm}$. The dimer formation and monomerization (reversion) dose-effect relations were determined by optical spectroscopy. The decrease of the OD value of the uracil thin layer at $288 \mathrm{~nm}$ was taken as a measure of the dimer formation, while the increase of the OD of a completely irradiated (until reaching the saturation level) uracil layer was the sign of the monomerization. The two processes in the UVB and the UVC range take place simultaneously, the individual characterization of the dimerization efficiency was performed from the initial slope of the dose-effect function and an action spectrum for dimerization was constructed. The reversion efficiency was found practically the same with all of the investigated wavelengths.

\section{P3.5 Studies of resistance to Mars UV conditions with extremely halophilic archaea}

*Sergiu Fendrihan, Helga Stan-Lotter

Department of Molecular Biology, Section of Microbiology, University of Salzburg, Billrothstrasse 11, A-5020, Salzburg, Austria

e-mail: sergiu.fendrihan@sbg.ac.at

The strong UV radiation demonstrated to be present at the Mars surface and the early Earth environment of 3.8 billions years ago showed that the potentially extinct or maybe extant micro biota need to be resistant to this kind of radiation. Exposure to space radiation of haloarchaeal species in the Biopan experiments was performed by Mancinelli et al. (1998) and they showed the high resistance of this organism to space conditions. Experiences with Halobacterium salinarum strain NRC-1 in liquid cultures exposed to UV radiation were performed by Baliga et al. (2004), which demonstrated the survival of this strain at an irradiation dosage of about $110 \mathrm{~J} / \mathrm{m} 2$.

We report some preliminary results of experiments within the HALOSPACE project (a subproject of ADAPT/EXPOSE; Principal Investigator: Dr. Petra Rettberg, DLR). The experiments were performed with the Martian UV Simulation Lamp, which produces similar UV radiation from $200-400 \mathrm{~nm}$ and an intensity as on the Mars surface (Kolb et al. 2004). The experiments were performed with strain Halococcus dombrowskii H4, a haloarchaeal strain isolated from Permian aged halite. Viability was determined by staining with the LIVE/DEAD kit, colony forming units and liquid culturing. The results showed that Hcc. dombrowskii survived (embedded in salt crystals), even at the dosage of $140.9 \mathrm{~kJ} / \mathrm{m}^{2}$.

Baliga, N. S., Bjork, S. J., Bonneau, R., Pan, M., Iloanusi, C., Kottemann, M. C. H., Hood, L. \& DiRuggiero, J. (2004). Systems level insights into the stress response to UV radiation in the halophilic archaeon halobacterium NRC-1. Genome Res. 14, 1025-1035.

Kolb, C., Abart, R., Bérces, A., Garry, J. R. C., Hansen, A. A., Hohenau, W., Kargl, G., Lammer, H., Patel, M. R., Rettberg, P. \& Stan-Lotter, H. (2005). An ultraviolet simulator for the incident Martian surface radiation and its applications, Int. J. Astrobiol. 4(3-4), 241-249.

Mancinelli, R. L., White, M. R. \& Rothchild, L. J. (1998). Biopan survival I: exposure of the osmophile Synechococcus sp (Nageli) and Haloarcula sp. to the space environment. Adv. Space Res. 22(3), 327-334.

\section{P3.6 Survivability and performance of cyanobacteria under simulated Martian UV-radiation

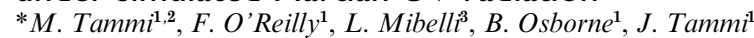 \\ ${ }^{1}$ University College Dublin, Dublin, Ireland \\ ${ }^{2}$ University of Turku, Turku, Finland \\ ${ }^{3}$ Universidad Central de Venezuela, Caracas, Venezuela}

Cyanobacteria are known to withstand extreme environmental conditions. Because of this, they have been proposed as candidates for biogenerative life support systems providing food, recycling water and producing oxygen for crew needs during planetary missions such as manned missions to Mars. In order to use cyanobacteria as the basis for life support systems, their responses to Martian conditions need to be known.

In this study we tested the survivability and performance of two edible cyanobacterial species - Arhtrospira platensis and a strain 
belonging to the genus Nostoc - to 20 minutes of that simulated Martian ultraviolet radiation. Treatment included all levels of UVradiation from UVC (200-280 $\mathrm{nm})$ through UVB (280-315) and UVA $(315-400 \mathrm{~nm})$ together with visible light $(400-700 \mathrm{~nm})$. Treatments were given using a rotating table in order to maximize the homogeneity of the treatments. The light environment was provided using two lamps, whose combined spectrum simulated that encountered on Mars.
During the treatments growth, cell numbers, amount of pigment concentration and photosynthetic activity were followed and monitored daily for eight days.

All cultures exposed to Martian levels of UV-radiation survived the treatment. When cultures were transferred to normal growth conditions, their growth rate and metabolic activity recovered fully.

\section{P4. Early life}

\section{P4.I Informational polymers in the primitive Earth: nonlinear analysis of archaea genomes and eukaryotic exons compared to computer-generated random sequences}

Giorgio Bianciardi

Department Patologia Umana e Oncologia, University of Siena, Via delle Scotte 6, 53100 Siena, Italy/Centro Studi di Esobiologia, Milano, Italy e-mail: gbianciardi@unisi.it

Informational polymers were born in the primitive Earth but nothing is known about the way(s) from which efficient prebiotic synthetic routes arose. However, in a possible scenario for the origin of life there was a time in which informational genetic polymers played primary roles. Possible 'fossils' of that primitive era (billions of years ago) may be considered the archaea domain (Howland 2000).

Nucleotide sequence data of archaea genes and codifying mRNA in eukarya (mammals) were obtained from the Institute for Genomic Research (TIGR) and the GeneBank library (http://cmr. tigr.org/tigr-scripts/CMR/CmrHomePage.cgi. and http://www.ncbi. nlm.nih.gov/). Random sequence data (white noise) were obtained from the algorithm by Press \& Teukolsky (1992). Nucleotide sequences were analysed as random walks, where each base represents a different step in a two-dimensional space; vice versa, the uniform and random distributed data points over the unit interval algorithm-generated were divided in 16 intervals to which A,C,G,T (U) letters were attributed.

Nonlinear parameters (relative LZ complexity, largest Lyapunov exponent, Hurst exponent, correlation dimension, entropy, BDS statistic, Manhattan and Euclidean fractal dimensions) of nucleotide sequences and computer-generated random sequences were evaluated making use of Chaos Data Analyser (Sprott \& Rowlands 1995) or Gates' formulation (fractal dimensions (Gates 1986)).

Our data show that the values of nonlinear parameters obtained from the archaea are lower than the values of randomly generated sequences $(p<0.01)$ and higher than the values from eukarya $(p<0.05)$. These data are in agreement with the ones by Weiss et al. (2000), showing a significant reduction of the Shannon entropy $(-1 \%)$ in protein sequences compared to random polypeptides. Our results suggest that in the primitive Earth informational polymers might be originated from slightly edited random strings and that during biologic evolution the distance from pure randomness increased. Deviation from pure randomness should be arisen from some constraints like the secondary structure of the biologic macromolecules, and, in eukarya, from chromatin interactions, according to the neoselectionist theory of genome evolution (Bernardi 2007).

Howland, J. L. The Surprising Archaea. Oxford University Press, 2000. Press, W. H. \& Teukolsky, S. A. (1992). Portable random number generators. Comput. Phys. 6, 522-524.

Sprott, J. C. \& Rowlands, G. (1995). Chaos data Analyser. Physics Academic Software.

Gates, M. A. (1986). A simple way to look at DNA. J. Theor. Biol. 119 319-328.

Weiis, O. et al. (2000). Information content of protein sequences. J. Theor. Biol. 206, 379-386.

Bernardi, G. (2007). The neoselectionist theory of genome evolution. PNAS 104(20), 8385-8390.

\section{P4.2 Viruses in the origin, evolution and panspermia} of life

* Matti Jalasvuori, Jaana K. H. Bamford

Department of Biological and Environmental Science, University of

Jyväskylä

Viruses are the most abundant genetically reproducing elements on our planet as they outnumber their hosts (the cells) in orders of magnitude. Current evidence suggests that viruses originated before the last universal common ancestor of cells, thus these parasites must have been an important factor in the evolution of the primitive life. But what is their role actually? Viruses are by their very nature selfish replicators, thus their need to exploit primordial resources might have given a logical pathway for natural selection to increase the complexity of proto-cells. Viruses in the form of an extra cellular threat to cells can be the acting force in the emergence of cell walls and therefore, indirectly, in the establishment of major domains of life and effective energy metabolism. Afterwards the evolutionary arms race between viruses and hosts might have caused the appearance of various different features, these being for example the second membranes over some bacteria.

Interestingly, if Panspermia of bacterial endospores has ever taken place, then viruses have relevance in understanding the further evolution of life on an alien planet. Our results suggest that the forming endospores of Bacillus thuringiensis only seldom take provirus genomes within, thus an endospore that revives on an alien planet after successful Panspermia probably does not bring any viruses to the founded ecosystem. The absence of viruses might fundamentally lead astray the evolutionary pathway from the one observed on Earth. The wide variety of different viruses on our planet also suggests that the life here is self-originated and not a result of interplanetary or interstellar voyage of micro-organisms.

\section{P4.3 Is the case against ribose proven?}

* Anthony R. Mellersh, Nigel J. Mason

Department of Physics and Astronomy, Open University, Walton Hall, Milton Keynes MK7 6AA, UK

The RNA world (Gilbert 1986) is an exciting prospect and there is persuasive evidence that life was based on RNA at some stage (Joyce 1989). The obvious route to ribose is through the 'formose' reaction. This yields a large number of sugars of which ribose is only a minor component (Decker et al. 1982). Furthermore ribose is unstable. For these reasons doubt is being cast on the role of ribose at the beginning of life, and alternative nucleic acid systems (ANAs) have been postulated. The idea is that such a system would initiate life before the emergence of RNA. The synthesis of ANAs is itself problematic, as is the mechanism of the transfer to RNA. In this poster we present an outline scheme for the synthesis and stabilization of ribose-5-phosphate, which could potentially overcome these problems and thus remove the need for a pre-RNA world.

Decker, P., Schweer, H. \& Pohlmann, R. (1982). J. Chromatography 244, 281-291.

Gilbert, W. (1986). Nature 319, 618.

Joyce, G. (1989). Nature 338, 217-224. 
P4.4 Shallow-water biolaminated sediments in a

\subsection{Ga-old chert from Barberton}

${ }^{*}$ Frances Westall ${ }^{1}$, Gisela Gerdes ${ }^{2}$, Axel Hofmann ${ }^{3}$

${ }^{1}$ Centre de Biophysique Moléculaire, CNRS, Orléans, France

e-mail: westall@cnrs-orleans.fr

${ }^{2}$ c/o Research Institute Senckenberg, Wilhelmshaven, Germany

${ }^{3}$ School of Geological Sciences, University of KwaZulu-Natal,

South Africa

Finely laminated, silicified volcanic sands and muds in the Josefsdal Chert from the $3.33 \mathrm{Ga}$ Kromberg Formation, Barberton Greenstone Belt are interpreted as shallow water, biolaminated deposits, similar to those found on modern mud-flat shores, e.g. Mellum in the North Sea. A finely structured, probably anaerobic photosynthetic microbial mat has previously been described from one of the bedding surfaces (Westall et al. 2006a,b). Recent field studies show that the Josefsdal Chert occurs at a stratigraphic level that is equivalent of the Footbridge Chert ( $3.333 \mathrm{Ga}$ ). The Footbridge Chert is interpreted as silicified deep water sediments (Lowe \& Byerly 1999). In contrast, detailed sedimentological investigations document the shallow water nature of the Josefsdal deposit. The sediments are fine-grained sands and muds that contain cyclical structures including fine laminations that are parallel, flaser, wavy or rippled. Channel bedding and lag deposits also occur. The ensemble of sedimentary structures indicates that the deposit formed in a back-barrier, intertidal flat environment.
The top layers of each cycle represent periods of equilibrium sedimentation (neither net erosion nor deposition). They are characterized by laminar concentrations of organic carbon. Generally there is much organic debris mixed in with the sediments. On the basis of the detailed study of the photosynthetic mat on one of the carbon-rich bedding planes (Westall et al. 2006a,b), and comparison with modern mud-flat environments, the organic-rich laminae are interpreted as probably representing the remains of microbial mats. We therefore conclude that these are biolaminated mud flat deposits.

Given formation of the Early Archaean terrestrial sediments in an anaerobic environment that is an excellent analogue for sediments formed on early Noachian Mars, these biolaminated deposits, with their cyclic, macroscopic structures and laminated concentrations of organic matter, are of interest for testing the kind of instruments that will be flown on in situ missions on Mars.

Lowe, D. R. \& Byerly, G. R. (eds) (1999). Geologic Evolution of the Barberton Greenstone Belt, South Africa, 329, GSA SP.

Westall, F. et al. (2006a). Implications of a 3.472-3.333 Ga subaerial microbial mat from the Barberton greenstone belt, South Africa for the UV environmental conditions on the early Earth. Phil. Trans. R. Soc. Lond. Series B 361, 1857-1875.

Westall, F. et al. (2006b). Geochemical profiling of an Early Archaean, littoral environment, photosynthetic microbial mat from the Barberton greenstone belt, South Africa. EANA abstract, Lyon.

\section{P5. Prebiotic chemistry}

\section{P5.I The fate of amino acids adsorbed on mineral matter}

*J.-F. Lambert, L. Stievano, I. Lopes

Laboratoire de Réactivité de Surface, UMR 7609, Université Pierre et Marie Curie, 4 Pl. Jussieu, 75252 Paris Cedex 05, France

Bernal's hypothesis, namely that mineral surfaces were instrumental in promoting the prebiotic condensation of small biomolecules to biopolymers, allowing such reactions to proceed in spite of unfavorable solution thermodynamics, has been the subject of much speculation and research activity. Here we summarize current knowledge on the topic from the point of view of surface science, and underline those points where further investigation is needed, concentrating on the case of amino acids polymerization to peptides. A lot is known on the molecular mechanisms of amino acids on oxides and silicate minerals (and very little on sulfide minerals). According to the nature of the amino acid and the adsorbent (with silica, alumina, and titania being the best studied), and the experimental conditions, electrostatic adsorption, covalent binding, adsorption by specific patterns of H-bonding, and by hydrophobic interaction, may all occur. Significant adsorption selectivities, and thus amino acids separation, are expected in some cases. Polymerization upon simple adsorption is a theoretical possibility, but has never been demonstrated due to a lack of experimental work. On the other hand, polymerization upon subsequent drying and heating at moderate temperatures has been demonstrated convincingly many times, in the frame of 'setting-and-drying' scenarii purporting to simulate climatic variability. 'Clean' polymerization, separated from further thermla degradation reactions, can definitely be obtained, but is unclear how to avoid the dead-end of cyclic dipeptides. Polymerization selectivities remain insufficiently explored so far, and it is desirable to devise experiments aimed at determining whether minimally functional peptide chains can be reasonably formed in minerals-supported systems. There is also a potential to follow thermal transformations in situ (without a desorption step) by techniques such as solid-state NMR.

\section{P5.2 Chemical evolution: amino acids at hot volcanic coasts}

*S. Fox, H. Strasdeit

Department of Bioinorganic Chemistry, Institute of Chemistry, University of Hohenheim, 70599 Stuttgart, Germany

It is generally believed that the earliest living cells emerged as a result of chemical evolution in the first billion years of Earth's history (Brack 2005). In this abiogenesis, amino acids might have played a key role. Some possible sources of amino acids can be considered (e.g. meteorites (Pizzarello 2004)). Thermal transformation of amino acids at hot lava coasts could have been the next step of chemical evolution towards new compounds. In the Hadean and early Archean, hot volcanic coasts were probably more abundant and the heat production was considerably higher than today. At such locations seawater evaporated and the remaining amino acid containing salt crusts experienced high temperatures. To model this scenario we thermolized an evaporated solution of an artificial sea salt mixture $(705 \mathrm{mmol}$ of $\mathrm{NaCl}, 15 \mathrm{mmol}$ of $\mathrm{KCl}, 15 \mathrm{mmol}$ of $\mathrm{CaCl}_{2}$, and $80 \mathrm{mmol}$ of $\mathrm{MgCl}_{2}$ ) that contained glycine ( $3 \mathrm{mmol})$, rac-alanine $(2 \mathrm{mmol})$, and rac-valine, rac-isovaline, racglutamic acid and a-aminoiso-butyric acid ( $1 \mathrm{mmol}$ each). The amino acid ratio used in this experiment is similar to the average ratio found in meteorites (Shock \& Schulte 1990). The thermolysis was conducted under an $\mathrm{N}_{2}$-atmosphere at $350^{\circ} \mathrm{C}$ and the volatile products were analysed by GC-MS. Among the decomposition products different Calkylated 1H-pyrroles were detected.

To demonstrate the importance of $\mathrm{Ca}^{2}+$ and $\mathrm{Mg}^{2}+$, a salt mixture containing only $\mathrm{NaCl}, \mathrm{KCl}$ and rac-alanine was prepared. Infrared and Raman spectra showed signals fitting to neat racalanine. In contrast, when $\mathrm{Ca}^{2}+$ and $\mathrm{Mg}^{2}+$ are present, the signals of the coordination compound $\mathrm{Ca}_{3} \mathrm{Cl}_{6}(\mathrm{Hala})_{2} \cdot 6 \mathrm{H}_{2} \mathrm{O}$ are found. The thermal behaviour of neat and $\mathrm{NaCl} / \mathrm{KCl}$ embedded rac-alanine is identical. In both cases only sublimation of rac-alanine and the formation of the cyclic dipeptide can be observed. The thermal behaviour of rac-alanine is strongly dependent on the coordination to metal ions, which therefore is of prime importance for the transformation, e.g. into pyrroles.

Based on these results it seems possible that on the early Earth pyrroles were formed from amino acids. As building blocks of important biomolecules (e.g. cytochromes, chlorophyll) pyrroles could 
have been the next step in chemical evolution. The following plausible reactions could have been the formation of oligopyrroles and their coordination to metal ions. The oligopyrroles and their metal complexes could have been used by primitive metabolizing systems and later modified into porphyrin-like macrocycles.

Acknowledgements: We thank the Deutschen Forschungsgemeinschaft for financial support. S. F. thanks the European Astrobiology Network Association (EANA) for a travel grant.

Brack, A. (2005). From the Origin of Life on Earth to Life in the Universe in: Lectures in Astrobiology, Vol. 1, ed. Gargaud, M., Barbier, B., Martin, H., \& Reisse, J., pp. 1-23. Springer, Berlin.

Pizzarello, S. (2004). Chemical evolution and meteorites: an update. Orig. Life Evol. Biospheres 34, 25-34.

Shock, E. L. \& Schulte, M. D. (1990). Summary and implications of reported aminoacid concentrations in the murchison meteorite. Geochim. Cosmochim. Acta 54, 3159-3173.

\section{P5.3 Protein subunits and the search of protein precursors \\ Franco Ferrari \\ Institute of Physics and CASA*, University of Szczecin, Poland}

Apart from the fold structures in proteins, which have been known for a long time, the existence of much smaller protein subunits has been recently suggested. Examples are the so-called motifs, which have a particularly stable structure and the loop subunits proposed by E. Trifonov and coworkers. Subunits of this kind seem to be intimately related to protein evolution and their detection inside proteins could provide invaluable information about the nature of primordial life. The detection of subunits in proteins poses many challenges from the technical point of view. In this poster the detection of subunits in crystallized proteins with the help of microcalorimetric techniques will be discussed.

\section{P5.4 How long can left and right handed life forms coexist? \\ Axel Brandenburg \\ Nordita, Stockholm, Sweden}

Reaction-diffusion equations based on a polymerization model are solved to simulate the spreading of hypothetic left and right handed life forms on the Earth's surface. The equations exhibit front-like behavior as is familiar from the theory of the spreading of epidemics. It is shown that the relevant time scale for achieving global homochirality is not, however, the time scale of front propagation, but the much longer global diffusion time. The process can be sped up by turbulence and large scale flows. It is speculated that, if the deep layers of the early ocean were sufficiently quiescent, there may have been the possibility of competing early life forms with opposite handedness.

\section{P5.5 Quantum origin of life}

Y. Toyozawa', *J. E. Dmochowski², M. Plaza ${ }^{2}$

${ }^{1}$ University of Tokyo (professor emeritus), Japan

${ }^{2}$ Kielce University of Technology, Faculty of Electrical and Computer Engineering, Al. 1000-Lecia Panstwa Polskiego 7D, 25-314 Kielce, Poland

The hypothesis that light coming from the Sun was at the very beginning of the life processes on the Earth is analysed: the temperature of the Sun, 5000-6000 K, 'seen' by the observer on the Earth facilitates 'non-equilibrium' - 'quantum' accumulation of energy by molecules. After excitation some molecules are subjected to photo-chemical reactions which could be described by the so-called configurationcoordinate diagram or Jahn-Teller effect. The latter phenomena are well known for solid-state physicists dealing with optical processes in solids. Energy accumulated in molecules could be used for 'selforganization' of molecules. The latter process could be facilitated in a warm thermal and water environment. The scenario given by
Y. Toyozawa in 'Optical Processes in Solids' seems to be very likely - 'Quantum' origin of life on the Earth.

\section{P5.6 Biforked state of a prebiotic microsystem: the intermediate step to living unit}

Vladimir N. Kompanichenko ${ }^{1,2}$

${ }^{1}$ Institute for Complex Analysis, 4 Sholom-Aleyhem St, Birobidzhan 679016, Russia

e-mail:kompanv@yandex.ru

${ }^{2}$ Department of Chemistry, University of California, 1156 High St, Santa Cruz 95064 CA, USA

According to the elaborated systemic conception (Kompanichenko 2004), organic microsystems of any kind that are considered as a prebiotic, have a chance to be transformed into primary living units only through the intermediate 'biforked' state. Bifurcation of a prebiotic microsystem may arise under non-equilibrium oscillating conditions in the environment when the microsystem simultaneously strives to transit into a new state and maintain the initial state with equal forces. In this case the striving of a prebiotic microsystem to develop into the different states maintains inner instability, while the balance between the opposite trends keeps its stability. A prebiotic microsystem at this paradoxical state of 'stabilized instability' acquires some properties which are at the background of biological organization: selfmaintaining heterogeneous structure, incessant fluctuations and rearrangement of molecules, exchange with the surroundings by matter and energy, the ability for dichotomy, etc. The most appropriate chemical composition of a prebiotic microsystem, which can be transformed into the intermediate biforked state, can be clarified through laboratory experiments in non-equilibrium oscillating conditions. It is argued that changeable conditions in hydrothermal systems and adjoining oceanic areas were suitable for such transformation of organic matter on the early Earth. The exploration of some present hydrothermal systems has allowed the characterization of the scale of thermodynamic minifluctuations that can be put in the background of advanced laboratory experiments on prebiotic chemistry: amplitudes $0.2-0.8$ bars (pressure) and $0.5-2{ }^{\circ} \mathrm{C}$ (temperature), period of oscillations $10-70$ minutes.

Kompanichenko, V. N. (2004). Systemic approach to the origin of life. Frontier Perspectives 13(1), 22-40.

\section{P5.7 Absorption of compounds of biological importance in solid surfaces and their relevance in terrestrial and extraterrestrial conditions \\ *López-Esquivel Kranksith Laura, Negrón-Mendoza Alicia, Ramos-Bernal Sergio \\ Instituto de Ciencias Nucleares, UNAM, Cd. Universitaria. Circuito Exterior, C.P. 04510, México}

Three prebiotic stages in the origin of life are recognized: inorganic, chemical and biological. Laboratory studies have been carried out simulating the chemical evolution in the possible conditions of primitive Earth. Experiments with various terrestrial solids (iron, clays, aluminium-silicates) have shown that they could act not only as surfaces of support, but also as catalysts. Studies of interstellar matter reveal the presence of organic molecules such as polycyclic aromatic hydrocarbons (PAH), fullerenes and possibly carbon nanotubes (CNTs), acetamide (a precursor of amino acids), simple amino acids and sugars. Irradiated clays, such as sodium montmorillonite, have been recognized to promote the synthesis of polymers and they act as non-covalent, catalytic crystalline insulators.

Carbon compounds called nanotubes (CNTs) are also able to function as supports and as catalysts, and they can also absorb specific compounds. The presence of CNTs in meteorites suggests that these solid tubular surfaces, could promote the formation of organic compounds of biological importance leading to exobiological evolution. In prebiotic chemistry it is important to investigate alternative routes for polymerization reactions and for the evolution of chemical compounds relevant for biology. 
In our present work the objective is to study the adsorption of compounds such as amino acids (Tyrosine (Y), Triptophan (W), Histidine (H), Aspartic acid (D)) onto silicates, clays and CNTs as a possible phase in the chemical evolution. We also study further the behavior of the compounds formed by amino acids adsorbed in these solid surfaces, in different conditions of $\mathrm{pH}$, concentration and levels of irradiation, simulating early Earth conditions.

We promote the hypothesis that amino acids, as biological units of proteins, were transported to the primitive Earth by meteorites (under chemical and physical adsorption) promoting later the catalysis of biological compounds on the Earth. The relevance of this work is to explain the possible contribution of solids (clay, silicates, CNTs) as promoters of polymerization and as shields for the adsorbed organic compounds against external sources of energy. We verify experimentally that the CNTs are better substrates for amino acids for maintaining their biological properties.

\section{P5.8 Characteristics of fluctuating conditions in the hydrothermal medium suitable for the origin of life *Vladimir Kompanichenko ${ }^{1,2}$, Polona Kralj ${ }^{3}$, Boris Fishman ${ }^{1}$, Konstantin Shlufman ${ }^{1}$, Efim Frisman ${ }^{1}$ \\ ${ }^{1}$ Institute for Complex Analysis, Birobidzhan, Russia \\ ${ }^{2}$ Department of Chemistry and Biochemistry, University of California at Santa Cruz, USA \\ ${ }^{3}$ Geological Survey of Slovenia, Ljubljana, Slovenia}

According to the elaborating systemic conception, the transition of prebiotic microsystems into simplest living units was stimulated by significant fluctuations of thermodynamic and physic-chemical parameters. Such fluctuating conditions are usual in hydrothermal systems including their outcrops in ocean or terrestrial groundwater aquifers. In this context characteristics of oscillations in natural hydrothermal systems can be used in advanced laboratory experiments on prebiotic organic synthesis under changeable conditions. To characterize a scale of the thermodynamic and physic-chemical fluctuations four hydrothermal systems were explored: Mutnovsky and Pauzhetsky springs in active volcanic Kamchatka region of eastern Russia, Mura (Slovenia) and Kuldur (Russian Far East) thermal fields situated in volcanically non-active regions. The temperature of water and the water-steam mixture in the Mutnovsky and Pauzhetsky fields ranges from less than $100{ }^{\circ} \mathrm{C}$ up to $240{ }^{\circ} \mathrm{C}$, water in Mura and Kuldur thermal basins is characterized with the highest temperature of $50-70{ }^{\circ} \mathrm{C}$. Data from monitoring pressure, temperature and some chemical parameters in wells of these fields were mathematically processed. Periods of long-range macrofluctuations of pressure and temperature in the Mutnovsky and Kuldur fields are 2-4.5 months, maximum amplitudes of temperature in the wells' orifices are $53{ }^{\circ} \mathrm{C}$ and $9{ }^{\circ} \mathrm{C}$ correspondingly, the maximum amplitude of pressure in the Mutnovsky field is 34 bars. Periods of minioscillations are from 10 to 70 minutes in the Mutnovsky, Pauzhetsky and Mura fields, average amplitudes of pressure are $0.2-0.7$ bars. Amplitudes of temperature and $\mathrm{pH}$ minioscillations in the Mura basin are about $1-2{ }^{\circ} \mathrm{C}$ and 0.2 correspondingly; there exists a strict positive correlation of temperature with $\mathrm{pH}, \mathrm{K}+, \mathrm{Na}+, \mathrm{Ca}^{2}+, \mathrm{HCO}_{3-}, \mathrm{SO}_{4}{ }^{2-}, \mathrm{Cl}-, \mathrm{F}-$, concentrations of $\mathrm{Mg}^{2}+, \mathrm{NH}^{4}+, \mathrm{CO}_{2}$ changing independently. The general conclusion is that minifluctuations of thermodynamic and physic-chemical parameters in hydrothermal systems are usual phenomenon. From time to time the parameters significantly change because of macrofluctuations that can be initiated by various causes (including earthquakes and volcanic eruptions). This changeable non-equilibrium medium is suitable for the geological Cradle of life on the early Earth. Kompanichenko, V. N. (2004). Systemic approach to the origin of life. Frontier Perspectives 13(1), 22-40.

\section{P6. Small bodies in the Solar System: Comets, Meteorites, Moons}

\section{P6.I Thermal history of micrometeoroids during the atmospheric entry \\ *G. Briani, S. Aiello ${ }^{1}$, A. Belleni ${ }^{2}$, L. Graziani ${ }^{1}$ \\ ${ }^{1}$ Department of Astronomy and Space Science, University of Florence, Largo Fermi 2, 50125 Firenze, Italy \\ e-mail:briani@arcetri.astro.it \\ ${ }^{2}$ Department of Civil Engineering, University of Florence, Via Santa Marta 3, 50139 Firenze, Italy}

Micrometeoroids (MMs) represent the largest mass flux of extraterrestrial matter to the present-day Earth and their flux was probably even larger during the heavy bombardment period. Micrometeoroids may have been the dominant source of organic carbon and may have contributed significantly to the delivery of water on the primitive Earth (Gounelle et al. 2005). Upon atmospheric entry micrometeoroids suffer a frictional heating, because of which their surface temperature rapidly reaches values as high as $1000 \mathrm{~K}$ or greater. At these temperatures organic compounds evaporate or decompose. Most of the current models pertaining to this topic assume the temperature of the particle as homogeneous through the whole volume. However, micrometeorites collected on the Earth's surface show evidences that thermal gradients developed during the atmospheric passage (Genge et al. 1997). The existence of these gradients may allow the survival of organic species in the interior of micrometeorites.

The high compositional and structural heterogeneity of micrometeoroids makes any exact treatment of the heat transfer extremely difficult. Moreover, MMs entering the atmosphere undergo transformations. Approximations are therefore unavoidable. In this work we propose a heuristic approach to the problem, i.e. we investigate and compare the effects of high temperature structure's alterations (dehydration, melting), and a temperature thermal conductivity's dependency. Assuming spherically symmetrical geometry and considering a MM composed by several concentric layers, we can solve the heat diffusion equation in 1D. Choosing the appropriate values of thermal parameters for the three resultant phases (compact, porous, melt) we can evaluate if temperature gradients appear, and if the internal layers of a MM can avoid heavy alterations. A critical issue is represented by the MMs surface temperature profiles used as boundary conditions for the heat diffusion equation. We have derived these time dependent profiles using a specifically designed numerical code that allows computation of the evolution of MMs temperature, velocity, mass and size, as a function of the initial values of speed, angle, radius and mass. The internal temperature profiles are computed along the MMs radius for different initial conditions, assuming MMs composed by silica. We adopted $900 \mathrm{~K}$ as the temperature for the passage from a compact to a porous structure (Flynn 1995), and $1880 \mathrm{~K}$ as Si melting temperature. Assuming the thermal conductivity of compact and porous phases varying with temperature as $1 / \mathrm{T}$, we use $1 \mathrm{~W} / \mathrm{m} / \mathrm{K}$ (Miyamoto et al. 1981) and $0.2 \mathrm{~W} / \mathrm{m} / \mathrm{K}$ (Bronshten 1983) as reference values (conductivity at $300 \mathrm{~K}$ ) for the two phases respectively. The magnitude of the temperature differences across the radius that result in MMs upon the crossing of the atmosphere depends on their entry speed and angle. As an example, our results show that for 400 and $500 \mu \mathrm{m}$ initial radius, with $12 \mathrm{~km} / \mathrm{s}$ entry speed and entry angle of $45^{\circ}$ with respect to the vertical, substantial gradients are produced. But if the entry angle is shallower (e.g. $80^{\circ}$ ) then these gradients do not appear.

This work was carried out as part of a broad theoretical and experimental program to study the passage of MMs through the Earth's atmosphere.

Bronshten, V. A. (1983). Physics of meteoric phenomena. D. Reidel Publishing Company.

Flynn, G. J. (1995). LPS Conference, XXVI.

Genge, M. J. et al. (1997). GCA 61, 5149-5162.

Gounelle et al. (2005). GCA 69, 3431.

Miyamoto et al. (1981). Proc. LPS 12, 1145. 


\section{P6.2 Dissociative recombination studies of} $\mathrm{CH}_{2} \mathrm{OH}+$ and $\mathrm{CD}_{2} \mathrm{OD}+$

${ }^{*}$ M. Hamberg ${ }^{1}$, W. D. Geppert ${ }^{1}$, R. D. Thomas ${ }^{1}$, V. Zhaunerchyk, F. Österdahl ${ }^{1}$, A. Ehlerding ${ }^{1}$, M. Kaminska ${ }^{2}, J$. Semaniak ${ }^{2}$, M. af

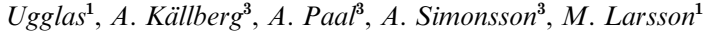

${ }^{1}$ Department of Physics, Stockholm University, Stockholm, Sweden

${ }^{2}$ Institute of Physics, Swietokrzyska Academy, Kielce, Poland

${ }^{3}$ Manne Siegbahn Laboratory, Stockholm, Sweden

Dissociative recombination (DR), the process where an electron recombines with a molecular ion and the excited neutral swiftly dissociates into neutral fragments is one of the most dominant reactions occurring in environments such as dark interstellar clouds, comas of comets and northern lights. Although common, DR is still considered to be 'unpredictable' and needs further investigation (Hamberg et al. 2007).

The Giotto spacecraft gathering samples from the nebula of comet Halley in 1986 was equipped with an ion mass spectrometer (IMS). The peak in the spectra corresponding to $31 \mathrm{amu} / \mathrm{e}$ is believed to be dominated by $\mathrm{CH}_{2} \mathrm{OH}+$. The ion is amongst the major ions in the inner coma, which makes the ion important for number density estimations of many neutral species in the same region (Haider \& Bhardwaj 2005).

Experimental studies of the branching ratios (BR) and the thermal reaction rate of the DR of the molecular ion $\mathrm{CH}_{2} \mathrm{OH}+$ and its deuterated isotopologue $\mathrm{CD}_{2} \mathrm{OD}+$ have been undertaken at the CRYRING storage ring located in Stockholm, Sweden.

Evaluation of the measured data yielded the following BR:

\begin{tabular}{llrl}
\hline Ion & Products & \multicolumn{1}{l}{$\mathrm{BR}$} & $\mathrm{Ch}$ \\
\hline $\mathrm{CH}_{2} \mathrm{OH}+$ & $\mathrm{COH} x+y \mathrm{H}+z \mathrm{H}_{2}$ & $92 \pm 2 \%$ & $\alpha 1$ \\
& $\mathrm{CH}+\mathrm{H}_{2} \mathrm{O}$ & $2.5 \pm 2 \%$ & $\beta 1$ \\
& $\mathrm{CH}_{2}+\mathrm{OH}$ & $5.5 \pm 1 \%$ & $\gamma 1$ \\
$\mathrm{CD}_{2} \mathrm{OD}+$ & $\mathrm{COD} x+y \mathrm{D}+z \mathrm{D}_{2}$ & $77 \pm 2 \%$ & $\alpha 2$ \\
& $\mathrm{CD}+\mathrm{D}_{2} \mathrm{O}$ & $5 \pm 1 \%$ & $\beta 2$ \\
& $\mathrm{CD}_{2}+\mathrm{OD}$ & $18 \pm 2 \%$ & $\gamma 2$
\end{tabular}

In the table the letters $x, y$ and $z$ are used to denote the possible variations of $\mathrm{H} / \mathrm{D}$ in the $\alpha$ channels. As can be seen, there is a distinct difference between the two isotopologues. Isotope effects within DR have been encountered before but tend in most cases to be of minor extent.

A comparison with the ions $\mathrm{DCO}+, \mathrm{CD}_{2} \mathrm{OD}^{2}+$ and $\mathrm{CH}_{3} \mathrm{OH}^{2}+$ shows a tendency towards $\mathrm{C}-\mathrm{O}$ bond fissure with increasing number of hydrogen/deuterium atoms.

Evaluation of the reaction rate coefficients yielded $7.0 \times 10^{-7}$ $(T / 300)^{-0.78} \mathrm{~cm}^{3} \mathrm{~mol}^{-1} \mathrm{~s}^{-1}$ for $\mathrm{CH}_{2} \mathrm{OH}+$ and $7.5 \times 10^{-7}$ $(T / 300)^{-0.70} \mathrm{~cm}^{3} \mathrm{~mol}^{-1} \mathrm{~s}^{-1}$ for $\mathrm{CD}_{2} \mathrm{OD}+$. The differences are considered to be within the error bars.

Hamberg, M. et al. (2007). Mol. Phys. 105(5-7), 899.

Haider, S. A. \& Bhardwaj, A. (2005). Icarus 177(1), 196.

\section{P6.3 Application of a minimum energy principle to the amino acids found in the carbonaceous chondrites \\ *Y. Ellinger ${ }^{1}$, M. Lattelais $^{1}, F$. Pauzat ${ }^{1}$, B. Zanda ${ }^{2}$ \\ ${ }^{1}$ Laboratoire de Chimie Théorique (LCT/LETMEX), Université Pierre et Marie Curie, Paris \\ ${ }^{2}$ Muséum National d'Histoire Naturelle, Paris}

Among the $\sim 70$ amino acids identified in carbonaceous chondrites, not a single one has been observed for sure in the interstellar medium. The question of the relative stability of these prebiotic compounds with respect to the other possible species of the same chemical formula is therefore an important question to be addressed. It is done here by means of 'state of the art' methods of quantum chemical simulations (DFT and Coupled Cluster calculations (CCSD(T)).

Considering all possible isomers of general formulas $\mathrm{CH}_{3} \mathrm{NO}$, $\mathrm{C}_{2} \mathrm{H}_{5} \mathrm{NO}$ and $\mathrm{C}_{3} \mathrm{H}_{7} \mathrm{NO}$ we found that the peptide bond containing species, namely, formamide (Chiaramello et al. 2005), acetamide and propanamide are the most stable. The recent discovery of acetamide by Hollis and co-workers (Hollis et al. 2006) is another illustration of the theoretical prediction that, when several isomers of the same chemical formula are possible, the most stable should always been observed. By contrast, we found that biological glycine (Lattelais et al. 2007), alanine and aminobutyric acid are far from being the most stable compounds of their respective families of isomers. It is generally considered that ion-molecule reactions are key processes of interstellar chemistry. In this context, we reconsidered all amino acids under their protonated forms. It appears that protonated glycine, $\beta$-alanine (more stable than biological alanine) and aminobutyric acids are the most stable compounds that can be formed.

On the other side, these compounds (in their neutral forms) are the most abundant amino acids found in the analysis of the carbonaceous matter in chondrites CI. These results suggest that amino acids could have been formed as protonated species in the interstellar medium and then incorporated in the meteorites in which they were neutralized. Protonated species should now be the next targets for astronomical search.

Chiaramello, J. M., Talbi, D., Berthier, G. \& Ellinger, Y. (2005). Int. J. Astrobiology 4, 125.

Hollis, J. M., Lovas, F. J., Remijan, A. J., Jewell, P. R. \& Ilyushin, K. I. (2006). Astrophys. J. 643, L25.

Lattelais, M., Ellinger, Y. and Zanda, B. (2007). Int. J. Astrobiology 6, 37 .

\section{P6.4 The limnological structure of Titan's hydrocarbon lakes and its astrobiological implication T. Tokano \\ Institut für Geophysik und Meteorologie, Universität zu Köln, Köln,} Germany

While the presence of extended hydrocarbon oceans on Saturn's moon Titan has been ruled out, several putative liquid hydrocarbon lakes have been recently detected in Titan's north polar region by the Cassini radar. Such lakes may contain organic sediments deposited from the atmosphere and promote further prebiotic chemistry driven by cosmic rays, by which more complex molecules such as nitrogen-bearing organic polymer or azides could be produced. Even the possibility of methanogenic life consuming acetylene and hydrogen or silane-based life in hydrocarbon lakes has been speculated. We investigate the physical properties of the lake and their temporal evolution under realistic Titan's climatic setting. It is shown that the lakes can undergo various evolutions depending on the initial composition and depth of the lake and hydrocarbon abundance in the near-surface atmosphere. Pure methane lakes have no chance of surviving since they evaporate, freeze up and eventually dry up. On the other hand, lakes filled with a mixture of methane, ethane and nitrogen are more stable and freezing or drying up can be prevented in most cases. These lakes undergo a seasonal cycle of thermal stratification and convective overturning, affecting the vertical exchange of dissolved gases and solids within the lake, between the lake and atmosphere and between the lake and lake sediments. Overturning may drive suspension and redistribution of solid acetylene on the lake bottom. These and other details are relevant in the consideration of Titan's 'prebiotic soup' as well as in the characterization of the lake as a putative habitat.

\section{P6.5 Non-destructive detection of biphosphammite and nickel-boussingaultite - two $\mathrm{NH}_{4}$ group containing minerals using vibrational spectroscopy} Adam Culka ${ }^{1,2}$

${ }^{1}$ Faculty of Science, Institute of Geochemistry, Mineralogy and Mineral Resources, Charles University in Prague

${ }^{2}$ Faculty of Science, Department of Inorganic Chemistry, Charles University in Prague

Raman spectroscopy has proven to be a very useful method for detection and analysis of various organic minerals and related substances. 
A wide range of materials of chemical or biological origin, such as organic minerals, biological pigments and fossil resins, can be easily detected by this method. These compounds and other biomarkers can frequently be used as proofs of pre-existing biosynthesis. This study focuses on two minerals containing $\mathrm{NH}_{4}$ group; biphosphammite $\left(\mathrm{NH}_{4}, \mathrm{~K}\right) \mathrm{H}_{2} \mathrm{PO}_{4}$ and nickel-boussingaultite $\left(\mathrm{NH}_{4}\right)_{2} \mathrm{Ni}, \mathrm{Mg}$ $\left(\mathrm{SO}_{4}\right)_{2} \cdot 6 \mathrm{H}_{2} \mathrm{O}$. Both are secondary minerals, whose formation is connected to life processes, the first being the 'cave' mineral from bat guano deposits. The aim of this study was to collect and interpret the Raman spectra of minerals that may be connected to the life processes on Earth.

In this study two spectroscopic techniques were used: Raman spectroscopy, being capable of non-destructive analysis of even a very small amount of sample, and infrared spectroscopy, which provides complementary data. Several excitation sources were used to minimize fluorescence, which may be caused by inorganic impurities.

In Raman and infrared spectra of both minerals, individual parts of mineral crystals give rise to various bands occurring in specific spectral regions. $\mathrm{NH}_{4}$ group is manifested in the spectral region between $3700-3000 \mathrm{~cm}^{-1}$ along with $\mathrm{OH}$ group (stretching vibrations) and at around $1650 \mathrm{~cm}^{-1}$ (bending vibrations). Bands corresponding to stretching and bending vibrations of both $\mathrm{SO}_{4}$ and $\mathrm{PO}_{4}$ groups were observed at lower wavelengths (e.g. 1144, 1134, 992, 989, $670 \mathrm{~cm}^{-1}$ ). Data obtained on materials studied here and on similar compounds can be useful for future investigations of extraterrestrial materials in the astrobiological context.

\section{P6.6 Raman spectroscopic non-destructive detection of organic minerals for exobiological studies \\ *J. Jehlicka1 ${ }^{1}$ H. G. M. Edwards ${ }^{2}$ \\ ${ }^{1}$ Charles University, Faculty of Science, IGMMR, Prague, Czech Republic}

${ }^{2}$ University of Bradford, Chemical and Forensic Sciences, Bradford, UK Organic minerals represent crystallized organic compounds occurring in rocks or mineral inclusions. They can be considered as a forgotten group of organic compounds in the geological record. The survival of organic minerals such as salts of carboxylic acids, terpenoid hydrocarbons and aromatic minerals in the geological or extraterrestrial record is important for an appreciation of the diversity of molecular compounds that need to be assessed in the search for life detection signatures not only in terrestrial but more importantly extraterrestrial scenarios.

Raman spectral signatures have been identified for different salts of carboxylic acids including mellite (hydrated aluminium salt of benzenehexacarboxylic acid), terpenoids, fichtelite (norabietane) and hartite (a-phyllocladane). Medium to high-temperature transformation processes can be documented by the presence of idrialite (complex polycyclic aromatic hydrocarbonaceous mineral), anthracene, phenenthrene, 9,10-antraquinone and phthalimide.

Raman microspectroscopy permits us to obtain information that is not easily obtained by other analytical methods. Its advantage consists in the possibility of evaluating the spectral characteristics non-destructively, using an objective lens of an optical microscope. This allows for the identification of individual parts of complex materials, organic compounds in the neighbourhood of minerals and micrometric inclusions in inorganic or organic minerals.

More attention can be given to Raman data application in the exobiological context - to detect several fragments or key structures that can be considered as having relevance to biogenesis. Detection of all kind of organic compounds and their radiation or heat transformation products could be of major importance. Obtained results suggest that further studies of organic minerals would be beneficial and appropriate for extension and inclusion in databases of biomolecular signatures which may have relevance to the adoption of Raman spectroscopic techniques for inclusion in instrumentation for life-detection and recognition of biological evidence in the geological records of extraterrestrial material.

\section{P6.7 Investigation of Europa biosignatures via hypervelocity impact physics \\ Katarina Miljkovic}

The Open University, Milton Keynes, UK

Europa is one of Jupiter's moons. It is important to explore because it is suspected of hosting some kind of life. Some of Europa's characteristics are related to the properties of a habitat, like the ones that can be found on Earth.

Europa (as many other Solar System bodies) doesn't have the atmosphere so the micrometeoroids that are attracted by gravity can easily reach the surface of the moon. Hitting the surface at certain velocity, micrometeoroids knock some material off. If the ejected material has sufficient kinetic energy, it can escape high enough above the surface of the moon and contribute to the dust cloud population around the moon. It is estimated that the dust spends quite some time in the cloud (and then either falls back and sticks to the surface or leaves Europa's gravity).

Therefore, if there is a dust detector flying through the dust cloud in the orbit around Europa, it could capture those dust grains (and analyse them too).

The surface composition will be written in the dust particles and this is the most important assumption! That includes any biomarkers (signs of life and biogenic activity in general) that exist on the surface. A previous mission indicated that the surface of Europa is mainly icy, but there are some reddish areas that are non-icy. That reddish material is most likely a type of sulphur-based hydrated salts and its origin is a bit mysterious. The sulphur may originate from Io (another of Jupiter's moons known by its volcanoes) or can be a product of geological activity in the Europa's core or can be sign for pre-biotic chemistry, even a by-product of extremophile like sulphur eating bacteria.

This poster presents current research on possible biomarkers on Europa and their detectability limitations as well as its application to a new dust detector design.

\section{P6.8 Stardust and Stardust Next missions} Johan Silen

Finnish meteorological institute, Helsinki, Finland

We present an overview of some results of the ongoing analysis of data retrieved by the Stardust spacecraft. The spacecraft was operated so well that it has been redirected to the comet Tempel-1 under the project name of Stardust Next. The goal of the mission is to acquire additional comet in situ dust observations and to take pictures of the Deep Impact mission impact crater. The spacecraft will arrive at the comet 14.2.2011.

\section{P6.9 Large icy satellites as possible sites for existence of biosphere}

Michael B. Simakov

Group of Exobiology, Institute of Cytology, RAS, St Petersburg, Russia All giant planets of the Solar System have a high number of satellites (61 of Jupiter, 52 of Saturn, known in 2003). A small part of them consist of very large bodies, quite comparable to planets of terrestrial type, but including a very significant share of water ice. Some of them have an atmosphere, e.g. the mass of a column of the Titan's atmosphere exceeds 15 times the mass of the Earth atmosphere column. Formation (or capture) of satellites is a natural phenomenon, and satellite systems definitely should exist at extrasolar planets. A hypothetical satellite of the planet HD 28185, with a dense enough atmosphere and hydrosphere, could have biosphere of terrestrial type (within the limits of our notion about an origin of terrestrial biosphere). 


\section{P7. Life in extreme conditions}

\section{P7.I Hydrocarbon rich extreme econiche} established in the deepest ice borehole at Vostok, East Antarctica

*I. Alekhina ${ }^{1,2}$, D. Marie ${ }^{3}$, J.-R. Petit ${ }^{2}$, V. Lukin ${ }^{4}$, V. Zubkov ${ }^{5}$, S. Bulat ${ }^{1,2}$

${ }^{1}$ Petersburg Nuclear Physics Institute RAS, St. Petersburg-Gatchina, Russia

e-mail:alekhina@lgge.obs.ujf-grenoble.fr

${ }^{2}$ Laboratory of Glaciology and Geophysics of Environment CNRS, Grenoble, France

${ }^{3}$ Station Biologique de Roscoff, Roscoff, France

${ }^{4}$ Arctic and Antarctic Research Institute, St. Petersburg, Russia

${ }^{5}$ St. Petersburg State Mining Institute, St. Petersburg, Russia

The main objective of this study was to analyse the original Vostok drilling fluid (DF) sampled from the borehole 5G-1 at different depths (up to $3600 \mathrm{~m}$ ) for bacterial content by $16 \mathrm{~S}$ ribosomal DNA sequencing and by this to evaluate the DF-containing borehole as a possible extreme econiche for hydrocarbon degrading bacteria. Additional objectives included gathering of information requested for the environmental assessment upon further exploration of Subglacial Antarctic Lake Environments (SALE) as well as that required for authentication of Vostok ice core findings (not discussed there).

The DNA analysis has led to identify six bacterial phylotypes. Two phylotypes dominated by clone numbers and recovered from the deepest and relatively warm borehole horizons $\left(>3400 \mathrm{~m} ;>-10{ }^{\circ} \mathrm{C}\right.$ ) were assigned to Sphingomonas - well-known degraders of aromatic compounds. Total cell concentrations there were about 102 cells $/ \mathrm{ml}$. By sequence similarity analysis and phylogenetic reconstruction one of two phylotypes, Sph. natatoria-related, proved to be of the same species with the isolate shown to degrade some mono-aromatic hydrocarbons while another one, Sph. sp. - with two isolates efficiently degrading phenanthrene and naphthalene both compounds present in original Vostok DF. The remaining four phylotypes were presumed to be contaminants due to their human or soil source.

Thus, the study showed that Vostok DF sampled from deep borehole horizons features two Sphingomonas phylotypes as indigenous DF-originating bacteria that fit the list of bacteria capable of degrading aromatic hydrocarbons and may expect to metabolize them in situ.

It seems that the new deeply ice-buried human-made extreme econiche is revealed in Antarctica. It is made of mostly aliphatic hydrocarbons but still containing aromatic, halogenated and fluorohydrocarbons, deeply buried beneath the ice (at $3000 \mathrm{~m}$ and deeper) and exposed with no light at temperatures higher than $-20^{\circ} \mathrm{C}$ and under high pressure (300 bars and higher) for a rather long time-period (up to 12 years). In general, such an icy environment could provide a habitat for anaerobic (low aerobic?) chemoorganotrophic piezophilic psychrophiles and specifically Sphingomonas are amongst them. The existence of such an econiche has distinct implications for searching for the life in organic-rich (harsh or toxic?) cold/icy extraterrestrial environments as existing elsewhere (e.g. Titan of Saturn) and indeed can pose the environmental issues upon exploring SALE.

\section{P7.2 Activity of a sulphate reducing bacteria community isolated from an acidic lake \\ *D. Wolicka ${ }^{1}$, A. Borkowski ${ }^{2}$ \\ ${ }^{1}$ Institute of Mineralogy, Geochemistry and Petrology, Faculty of Geology, Warsaw University, al. Zwirki i Wigury 93, 02-089 Warsaw, Poland \\ e-mail:d.wolicka@uw.ed \\ ${ }^{2}$ Institute of Agricultural Microbiology, Department of Soil Sciences, Faculty of Agriculture and Biology, Warsaw Agricultural University, Nowoursynowska 159, 02-776 Warsaw, Poland \\ Sulphate-reducing bacteria (SRB) play a significant role in the environment, mainly because of their ability to metabolize organic}

matter. It is estimated that they participate in the degradation of over $50 \%$ of the organic matter present in different environments.

The aim of this study was the isolation, selection and search activity of SRB from an acidic lake. The research was focused on the activity of an anaerobic bacterial community capable of reducing sulphate in an extreme environment at a low $\mathrm{pH}$ value of about 3 .

Anaerobic communities of microorganisms were isolated from the 'Purple Pond' acidic lake, located in the central part of the Sudety Mountains (SW Poland), within an abandoned pyrite mine near the village of Wiesciszowice.

The bacterial communities were isolated on media containing ethanol and/or lactate as the sole carbon source on a Postgate or/and minimal medium. Both media were enriched with sulphates. The microorganisms were isolated from sediments, multiplied using the method of microcosms and incubated at room temperature of about $20{ }^{\circ} \mathrm{C}$. Resazurin in the concentration $0.001 \mathrm{~g} / 1$ was added to all cultures as an indicator of redox conditions in the medium.

A total of eight cultures were established. In general, the high activity of SRB was observed only on a Postgate medium with lactate. Later the products of biotransformation of sulphate during the culture growth were analysed. During the analysis both microbiological and geochemical methods were applied, such as diffractometric and SEM analysis, thanks to which the morphological contents and chemical composition of the resulting products could be determined.

The results of the research show that biogenic sulphate reduction, as well as oxidation of sulphide, are the main processes in an acidic lake. A very important stage in the biogeochemical sulphur cycle is the biological reduction of sulphate by SRB. Moreover, studies on the chemical composition of products of bacterial activity can aid a better understanding of geomicrobiological processes in a low $\mathrm{pH}$ environment.

\section{P7.3 Biosignatures in pillow lava alteration rims (Atlantic Ocean): implications for biogenecity and their detection}

B. Cavalazzi ${ }^{1},{ }^{*} F$. Westall ${ }^{2}$, R. Barbieri ${ }^{1}$

${ }^{1}$ Dipartimento di Scienze della Terra e Geologico-Ambientali, Università di Bologna, Bologna, Italy

${ }^{2}$ Centre de Biophysique Moléculaire-CNRS, Orléans, France

Microbes living within modern oceanic crusts (bioalteration) expand the range of known extreme microbial environments. Cryptoendolithic microorganisms can bioerode fractures in the glassy selvages of pillow lavas leaving behind traces of microbial life as microtubules. Mineralfilled microtubules recovered in the Archean upper oceanic crust are now claimed as a biosignature for life on early Earth and perhaps Mars. Depending on thermal conditions, however, abiotic processes can be involved in alteration features observed in basaltic glass of submarine pillow lavas.

Here we present the preliminary results of the investigation of features occurring within pillow lavas at the alteration front. Samples come from the upper portion of altered, recent pillow lava samples collected during the scientific cruise SWIM 2004, at station 29 by R/V Urania, Atlantic Ocean - Gulf of Cadiz. A multi-analytical approach (optical and confocal microscope, and ESEM equipped with EDS) was then used for testing the biogenicity of the filamentous morphologies and their $3 \mathrm{D}$ reconstruction. In summary, the biogenicity of the filamentous morphologies in the studied material is based on: (i) the evidence of morphological structures at different magnifications and their comparison with modern and fossil analogues; (ii) the geological and environmental context that support the syngenicity of the microbial-like morphologies; the petrographical analyses and microbe-mineral interactions that suggest metabolic pathways of the microbial-like morphologies; (iii) the X-ray mapping and spot analyses of the elemental distributions that demonstrate significant carbon 
concentrations within the filamentous morphologies. This study documents the utility of confocal microscopy for detailed investigations of subsuperficial structures and morphologies of microbial origin.

Acknowledgments: The Authors would like to thank Nevio Zitellini (ISMAR-CNR, Bologna), PI of the SWIM 2004 (ESF project: 'Earthquake and Tsunami hazards of active faults at the South West Iberian Margin: deep structure, high-resolution imaging and paleoseismic signature'), for providing the samples used in this investigation. Thanks are due to Gilles Breuzard, Centre de Biophysique Moléculaire, CNRS (Orléans), and Roberto Braga (Università di Bologna) for their support during the confocal microscope observations and for the interpretation of petrographical data, respectively. This work was partially supported by CNR-CNRS International Program 2006-2007.

\section{P7.4 Mycobacterium species in geothermal areas from Yellowstone National Park \\ ${ }^{*}$ Ricardo Santos $^{1,2}$, João Fernandes ${ }^{1}$, Fernanda Oliveira ${ }^{1}$, Manuela Cadete ${ }^{1}$ \\ ${ }^{1}$ Instituto Superior Técnico, Laboratório de Análises, Av. Rovisco Pais 1049-001 Lisboa, Portugal \\ e-mail: ricardosantos@ist.utl.pt \\ ${ }^{2}$ School of Biological Sciences, MBC, Queen's University Belfast,} Belfast, Northern Ireland

Mycobacterium is one of the oldest bacterial genera. Although some mycobacteria are agents of diseases in man and other animals, most mycobacteria are free-living saprophytes that form part of a balanced microbial community in natural habitats such as soil and water.

Mycobacteria optimal growth conditions are mild temperature and acidic $\mathrm{pH}$ but some species are able to survive, in hot water, and in extreme acidic conditions. Recently, one study isolated several sequences belonging to mycobacteria in pore water extracted from rocks collected, in extreme environments, in Norris Geyser Basin, Yellowstone National Park, but no cultivable microbes have been recovered. It was, therefore, important to recover cultivable microbes from these environments to better understand physiological and genetic adaptations that allow these usually mesophilic species to survive in geothermal environments and to understand their influence in the ecology of these extreme environments. Water samples were collected from different thermal locations in Yellowstone National Park. Samples were analysed by culture-based, fluorescent microscopy and molecular biology methods. Mycobacterium species have been isolated in several locations in Yellowstone National Park, mainly along Norris Geyser Basin. Thermal resistance profiles were made to confirm the origin of isolated strains. Further studies are being conducted to better understand temperature-resistance mechanisms that allow these microorganisms to survive in such extreme environments.

\section{P7.5 Life in mud volcanoes}

A. Denzil ${ }^{1}$, S. Haque ${ }^{1 *}$, H. J. Lehto ${ }^{2}$, A. Ramsubhag ${ }^{1}, B$ Wilson $^{1}$

${ }^{1}$ The University of the West Indies St Augustine Campus, Trinidad and Tobago, The West Indies

${ }^{2}$ Tuorla Observatory and Dept of Physics, University of Turku, Finland Geochemical data collected from numerous under water mud volcanoes such as those along the Mediterranean Ridge and Lake Baikal indicate a diversity of bacterial life and microorganisms. This experiment involves the investigation of three mud volcano sites in Trinidad. They are located at Digity, Devil's Woodyard and Piparo. Sediment samples were extracted from each site at depths of 0.1 metre and 1 metre. Samples were cultured under aerobic, anaerobic, methane aerobic and methane anaerobic conditions. Other types of organisms could have been analysed, however for this experiment only the types listed above were investigated. After a one-week incubation period the cultures were observed to have active bacteria colonies present. We will characterize these.

At the Isidis Planitia Basin on Mars mound like features have been observed on the surface. These could be of a result of the expulsion of fluids and gases from sediment layers similar to mud volcanoes. The implication is that if methane gas and subsurface fluids was expelled from these features onto Mars' surface, organisms may be capable of survival as they do in the mud volcanoes of Earth.

\section{P7.6 Fungi in astrobiology \\ Tapani Yli-Mattila} Department of Biology, University of Turku, Finland

Green photosynthetic plants form the basis of the energy and organic compounds required for making a planet or a space ship capable of surviving. But plants cannot survive without other organisms. Fungi have several important roles in the ecosystems. They are essential in decomposing and circulating organic material and compounds. They are also important symbionts in mycorrhizas, which assist nutrient uptake in plants. In lichens the role of fungi in nutrient uptake and protecting the symbiotic algae against UV light is essential. In addition, fungi are the most important group of organisms causing plant diseases. On the other hand fungal spores are very resistant against the conditions present in space and might survive in meteorites and space ships from one planet to another planet. Thus, astrobiologists have to take fungi into consideration, when they are investigating, what kind of organisms would be necessary for ecosystems outside earth.

\section{P7.7 Growth and survival of coloured fungi in space} ${ }^{*}$ Gomoiu Ioana ${ }^{1}$, Piso Marius ${ }^{2}$, Hasegan Dumitru ${ }^{3}$,

Evangelia Sarantopoulou ${ }^{4}$

${ }^{1}$ Institute of Biology, Bucharest, Romania

${ }^{2}$ Romanian Space Agency, Bucharest, Romania

${ }^{3}$ Institute of Space Science, Bucharest, Romania

${ }^{4}$ National Hellenic Research Foundation, TPCI, Athens, Greece

The project 'Growth and survival of coloured fungi in space' is based on the experiments both on Earth and in Space inside of microcapsules made by experts from the Romanian Institute of Space Science. Microbiology activity studies and Atomic Force Microscope imaging of spores will be performed in the Romanian Institute of Biology and National Hellenic Research Foundation, TPCI, respectively. The fungal species chosen for experimental studies belong to different genera and were selected as organic material decomposers. Studies with Ulocladium sp cultures and spores belonging to different fungal species will be done both in normal conditions on Earth and in the ESA EXPOSE facility loaded on the SOYUZ-taxi - inside of ISS in order to determine the effect of microgravity and cosmic radiation on the colonial growth and survival rates of spores. Fungal species containing melanin are protected against UV rays. Survival of black fungal spores in space is highly important for the theory of panspermia and for preventing contamination during interplanetary travelling. Results can be taken into consideration for calculation of the survival and possible proliferation of potential contaminants transferred from Earth to the spacecraft. It would be possible to explain if terrestrial fungi are able to survive, germinate and replicate to make new hyphae under extreme conditions.

According to exobiology, life appeared in extreme conditions and the project will give some answers about how life can be maintained and for how long in extreme conditions existing in space.

The main tasks of the project are:

- Measurements of colonial growth of Ulocladium sp., being 0-4 days old, during spaceflight. Using proper software the diameters of colonies and the length of active growth area will be measured. In addition the dynamic of sporulation during spaceflight will be done by comparing different photographic images. The same biological features are measured on Earth.

- Spores exposed for a short and a long time in ISS will be examined for their survival, germination and capacity to make new colonies. Some of the spores belonging to chosen species can be responsible for contamination of spacecraft during space missions. Phenotypic and metabolic expressions of mutagenesis will be put in evidence. 
- Observation of the spreading and adhesion of spores on different substrates in microgravity conditions. This process can be put in evidence by optical microscopy as a proof of spore spreading in microcapsule. The above measurements will be performed in microcapsules placed on Earth.

- Optical microscope and AFM images of spores and hyphae exposed at cosmic radiation and microgravity will be compared with the corresponding ones under normal conditions (laboratory) in order to reveal the changes induced by the above parameters.

\section{P7.8 Effects of the ISS low Earth orbit related environment on the transcriptome and proteome expression in the ESA Life Support System bacterium Rhodospirillum rubrum ATCC25903 \\ $* F$. Mastroleo ${ }^{1,2}, N$. Levs ${ }^{2}$, R. Benotmane ${ }^{2}, F$. Vanhavere ${ }^{2}$, A. Janssen $^{2}$, L. Hendrickx ${ }^{2}, M$. Mergeay ${ }^{2}, R$. Wattiez ${ }^{1}$ \\ ${ }^{1}$ University of Mons-Hainaut (UMH), Laboratory for Proteomics \& \\ Protein Biochemistry, Mons, Belgium \\ ${ }^{2}$ Belgian Nuclear Research Center (SCK•CEN), Laboratory for \\ Molecular \& Cellular Biology, Mol, Belgium}

MELiSSA (Micro-Ecological Life Support System Alternative) is a bioregenerative life support system for future space flights developed by the European Space Agency. It consists of interconnected bioreactors targeting the total recycling of organic waste into oxygen, water and food. Within the MELiSSA loop, the purple non-sulphur $\alpha$ proteobacterium R. rubrum is used to convert fatty acids, released from the upstream raw waste-digesting reactor to $\mathrm{CO}_{2}$ and biomass.

The functional stability of such bioreactors under space flight conditions is of paramount importance for the maintenance and crew biosafety during future long-term missions. Therefore, the physiological and metabolic changes induced by space flight were investigated for R. rubrum. The bacterium grown on solid medium during two different 10-day space flights to the ISS (MESSAGE2, BASE-A experiments) were compared to cells grown on Earth $1 \mathrm{~g}$ gravity or simulated microgravity and normal Earth radiation or simulated space flight radiation conditions in order to relate each single stress to its respective cellular response. For simulating the radiation environment, pure gamma and neutron sources were combined, while simulation of changes in gravity where performed using the Random Positioning Machine and the Rotating Wall Vessel technology. Transcriptome analysis using $R$. rubrum total genome DNA-chip showed up-regulation of genes typically involved in oxidative stress response after a 10-day mission inside the ISS, without loss of viability. As an example, bacterioferritin, alkyl hydroperoxide reductase, thioredoxin reductase genes are least two fold induced although the radiation dose experienced by the bacterium $(4 \mathrm{mSv})$ is very low compared to its radioresistance $(\mathrm{D} 10=100 \mathrm{~Sv})$. Next to this, groups of genes related to chemotaxis, flagellum formation and nitrogen metabolism were also differentially expressed. Transcriptomic data were combined to LCMS/MS proteomic data collected from the same $R$. rubrum samples since parallel profiling of mRNA and protein on a global scale could provide insight into metabolic mechanisms underlying complex biological systems.

Ground simulations suggest that the metabolic changes in the $R$ rubrum space flight samples would be mainly induced by ionizing radiation but the effect of changes in gravity should not be neglected. These results indicate the importance of studying the effect of low doses of ionising radiation on microorganisms.

\section{P7.9 Astrobiological experiments on Foton-M3}

\section{R. Demets}

ESTEC, Noordwijk, The Netherlands

Last month, the Foton-M3 unmanned recoverable capsule completed a 12-day mission in space. Included in the payload were twelve astrobiological experiments provided by ESA. Ten experiments, fitted together in the Biopan facility, were exposed to the harsh space environment. Two more were mounted in the heat shield of the Foton capsule, being directly exposed to the re-entry environment. In total, 15 biological species were involved including yeast, tardigrades, rotifers, lichens, nematodes and bacteria. An overview will be presented of the mission campaign, the experiment environment and the payload performance.

\section{P7.I0 HALOSPACE - testing the survival of Halococcus dombrowskii in the ESA EXPOSE facility at the International Space Station \\ *Tatjana K. Polacsek, Helga Stan-Lotter \\ University of Salzburg, Division of Molecular Biology, Department of \\ Microbiology, Salzburg, Austria \\ e-mail:tatjana.polacsek@sbg.ac.at \\ Halococcus dombrowskii, a halophilic archaeon, is used in the} HALOSPACE project (a subproject of ADAPT/EXPOSE) to investigate its survival strategies under stress, caused by cosmic and Solar UV radiation and desiccation. A strongly pigmented wild type strain and cells with reduced pigmentation will be exposed to space conditions using the ESA facility EXPOSE on the International Space Station (ISS). One aim of the project is to gather information if pigmentation has any influence on protecting organisms against radiation in outer space.

This work describes attempts to reduce the cells' pigmentation and the preparation of the quartz discs onto which small samples of Hcc. dombrowskii $\mathrm{H} 4$ are transferred to form single crystals. For the purpose of pigment reduction different growth media [Modified Growth Medium (MGM 25.7\%; according to the Halohandbook by Dyall-Smith), M2 Sea Salt Medium (22.2\% with Instant Ocean/Sigma) and others] as well as chemical substances [diphenylamine (DPA), glycerol] were used. Cultures were harvested by centrifugation, washed with $\mathrm{TN}$ buffer ( $50 \mathrm{mM}$ Tris, $4 \mathrm{M} \mathrm{NaCl}, \mathrm{pH}$ 7.4) and the pellets kept at $-70{ }^{\circ} \mathrm{C}$. Pigment extraction was carried out under nitrogen atmosphere with acetone/methanol $(1: 1, \mathrm{v} / \mathrm{v})$. For pigment analysis thin layer chromatography (TLC) with different solvents [acetone in benzene (50:50); benzene/acetone (65:35); chloroform/methanol (93:7)] were used. Pure beta-carotene served as a reference. The exact composition of the overall pigments of Hcc. dombrowskii is not yet known; however, beta-carotene was detectable as a major TLC spot. Furthermore a pigment scan with a Novaspec Plus photometer (General Electrics) was carried out, demonstrating the presence of the typical bacterioruberin and carotenoid peaks and showing a reduced absorption of the less pigmented strain.

For experimental verification tests (EVT), $40 \mu$ of suspension of Hcc. dombrowskii cells with reduced pigmentation were transferred to quartz discs, dried and transported to the German Aerospace Center (DLR) Köln, Germany. After having completed the test series, the discs were returned to the laboratory and evaluation of survival was carried out by determining the colony forming units (CFU) and staining for viability, using LIVE/DEAD BacLight KitTM (Bacterial Viability Kit, Molecular Probes).

\section{P7.II Habitats on the external parts of the International Space station? \\ C. Muller}

Belgian Institute for Space Aeronomy and B.USOC, Brussels, Belgium While extensive studies of the microbial communities are performed inside manned spacecrafts, the exterior has generally been considered as a sterile world where there was no concern for toxicity or for microbial contamination. However, as construction work accelerates, frequent EVA's and arrival of supply spacecrafts bring constantly biologically rich dust, liquids and gases in contact with the external skin of the station

In the process of studying the implantation of external payloads on the space station, the problem of contamination has been addressed essentially up to now from the point of view of optical cleanliness. This 
point is quite fundamental for ultraviolet sensors as a normally transparent deposit might be photo-dissociated and leave an opaque coating on lenses and mirrors. The question of the toxicity or of biologically nutritive value of the contaminants has never been a concern on the exterior of the station while of course; it is controlled at the maximal possible extent for the pressurized parts of the station.

A comparison will be made between the space shuttle and the station. The development of new habitats as the station grows and new shielding devices are added will be considered. The necessity of introducing new precautions for the protection of future external biological exposure experiments will be considered.

\section{P7.12 Cleaning-resistant Cupriavidus and Ralstonia} bacteria contaminating spacecrafts and the ultra clean rooms they are assembled in

*Natalie Leys ${ }^{1}$, Annik Dams $^{1}$, Albert Bossus ${ }^{2}$, Ann Provoost $^{2}$, Kasthuri Venkateswaran $^{3}$, Max Mergeay ${ }^{1}$

${ }^{1}$ Laboratory of Radiobiology \& Microbiology, Belgian Nuclear Research Centre (SCK•CEN), Boeretang 200, 2400 Mol, Belgium

${ }^{2}$ Laboratorium voor Moleculaire Biologie in Milieu en

ProcessTechnologie (MPT), Vlaamse Instelling voor Technologisch

Onderzoek (VITO), Boeretang 200, $2400 \mathrm{Mol}$, Belgium

${ }^{3}$ Biotechnology \& Planetary Protection Group, Jet Propulsion

Laboratory (JPL), California Institute of Technology, Oak Grove Drive 89-2, 91109 Pasadena, California, USA

Background: 'Planetary Protection' is preventing microbial contamination of both the target planet and the Earth when sending spacecrafts on interplanetary space missions. It is important to preserve the natural conditions of other planets and to not bring with robots 'earthly microbes' (forward contamination) when looking for 'spores of extra terrestrial life'. Spacecrafts and the ultra clean rooms they are assembled in, are routinely monitored for microbial contamination. It was shown that the floor, air and surfaces of such spacecraft assembly rooms often contain Cupriavidus and Ralstonia bacteria. These bacteria not only contaminated the clean rooms but have also been found priorto-flight on surfaces of space robots such as the Mars Odyssey Orbiter and even in-flight in ISS cooling water and Shuttle drinking water (unpublished).

Aim: In this study, several Cupriavidus and Ralstonia strains isolated from space craft assembling rooms and spacecrafts were characterized and analysed in detail.

Results: The analysis showed that all the Cupriavidus and Ralstonia clean-room isolates are able to use a wide variety of substrates as carbon sources, including ethanol and acetone. In addition, they all have accumulated moderate resistances to an extraordinary collection of physical and chemical antimicrobial agents. Some of the test strains were able to form biofilms on plastic and metal materials used for space robots, a nutritional and resistance advantage. Surprisingly, all strains were found to be also resistant to a whole range of heavy metals, Moreover, the resistance properties may be encoded in 'mobile' DNA fragments as several large plasmids were detected in these strains. This phenotype is very similar to that of well-known strain Cupriavidus metallidurans $\mathrm{CH} 34 \mathrm{~T}$, which carries a big collection of heavy metal resistance genes on two large plasmids.

Conclusions: The absence of a clear selection or need for heavy metal resistance for bacteria to survive in a clean-room environment, suggests that these adaptations have been acquired in the past in their original environmental habitat, probably the contaminated soil surrounding the spacecraft assembling facilities. They were possibly introduced via shoes and equipment. The accumulation of a wide variety of moderate resistances, originally needed to survive in the poor, repeatedly contaminated and continuously changing industrial soil environment, has given these bacteria the advantage to survive different acute and chronically 'life-threatening' stresses more easily and to adapt to new harsh man-made environments such as ultra clean spacecraft assembly rooms.

Future work: The further study of the source, introduction and survival strategy of these Cupriavidus and Ralstonia bacteria contaminating spacecraft assembly rooms and spacecrafts, is of importance to improve contamination prevention, monitoring and disinfection tools for the future.

\section{P7.I3 An introduction to planetary protection Euan Monaghan University of Kent, Canterbury, UK}

The concept of planetary protection is a key one within the field of astrobiology. Its aim is to promote the methods and protocols that strive to preserve any extant or extinct life on planets other than our own and to prevent cross-contamination. This poster describes those methods and protocols and includes mention of previous lapses in those same processes. The scientific and ethical reasoning behind the whole endeavour is discussed, as is its potential culmination. The poster is aimed at those with a non-astrobiology background.

\section{P7.I4 Raman spectroscopy study of extremophile organisms and their relationship with minerals and rocks}

*Susana E. Jorge Villar ${ }^{1,2}$, Howell G. M. Edwards ${ }^{2}$

${ }^{1}$ Area de Geodinamica, Facultad de Humanidades y Educación, University of Burgos, Burgos, Spain

e-mail:seju@ubu.es; susanajorgevillar@hotmail.com

${ }^{2}$ Centre for Astrobiology and Extremophile Research, Analytical Centre, School of Life Sciences, University of Bradford, Bradford BD7, 1DP, UK Extremophile micro-organisms have developed different strategies to withstand hazardous environmental conditions. The production of organic compounds, such as sun-screen pigments or anti-freeze substances, is the first strategy, but in the worse situations, such as in very cold deserts (Arctic and Antarctic areas), hot deserts (Atacama, Negev), acid, basic or saline waters, their interaction with rocks could be vital.

The most widespread practice is to migrate inside a crack or a porous rock; several millimetres or, in some cases, centimetres of rock play an effective radiation screen role. Humidity, temperature, salinity or nutrients can be different from outside and create a microenvironment more favourable for life.

The interaction micro-organism/rock is not only limited to the use of rocks as a habitat but modifications in the closest mineralogy have also been observed in many endoliths (inside the porosity) and chasmoliths (inside a crack). These changes include mineral mobilization as well as mineral-phase transformations.

For the analysis of those interactions we have used the Raman spectroscopy. This is a non-destructive analytical technique that allows us to study either organic or inorganic compounds without chemical or physical manipulation (except to break the rock to show the microorganisms). The identification is unambiguous since is sensitive to structure and composition and then, isomorphous and polymorphous molecules can be distinguished.

Extremophile micro-organisms have been considered analogues to possible extinct or extant Martian life (if sometimes appeared); moreover, Raman spectroscopy can be included as a part of a suite of analytical instruments to be sent to Mars for looking for life. The study of a wide range of extremophiles and their protective strategies in different hazardous environmental conditions and the elaboration of an exhaustive data-base is an important requisite for searching for life in extra planetary surfaces. 


\section{P8. Lithopanspermia}

8.I LITHOPANSPERMIA: test of interplanetary transfer and re-entry process of epi- and endolithic microbial communities in the FOTON-M3 Mission

${ }^{*}$ R. de La Torre ${ }^{1}$, L. G ${ }^{a}$ Sancho $^{2}$, G. Horneck ${ }^{3}$, Petra Rettberg ${ }^{3}$,

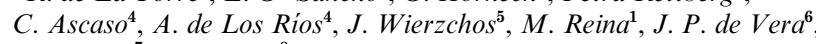
C. Cockell ${ }^{7}$ R. Demets ${ }^{8}$

${ }^{1}$ INTA, Spanish National Aerospace Establishment, Madrid, Spain

${ }^{2}$ Universidad Complutense Madrid, Facultad de Farmacia, Madrid, Spain

${ }^{3}$ German Aerospace Center, Cologne, Germany

${ }^{4}$ CSIC, Center of Environmental Sciences, Madrid, Spain

${ }^{5}$ Universidad de Lérida, Lérida, Spain

${ }^{6}$ Universität Düsseldorf, Düsseldorf, Germany

${ }^{7}$ OPEN University, Milton Keynes, England

${ }^{8}$ ESA-ESTEC, Noordwijk, The Netherlands

This experiment has been designed to test experimentally two steps of the Lithopanspermia hypothesis on board the FOTON-M3 satellite: (1) Travel through space (step 2) on board of Biopan-6, and (2) Capture and landing on another planet (step 3), integrated in ESA's Stoneexperiment. Step 1 (escape process from the planet of origin by an impact of an asteroid or a meteorite), is currently studied within a DFG-project (Stöffler et al. 2007; Horneck et al. 2007). Intercomparison of the survival capacity of different epi- and endolithic microbial communities (prokaryotic and eukaryotic systems) will be done after 10 days exposure to harsh space conditions in low Earth orbit (200-300 km altitude). Tests of step 2 of Lithopanspermia include the following bipolar epilithic lichen species (on their natural rock substrate): Rhizocarpon geographicum and Xanthoria elegans, which have already demonstrated high survival rates after space exposure in the experiment Lichens onboard of the Foton-M2 mission (de la Torre et al. 2007; Sancho et al. 2007). The purpose of this follow-up experiment is to also check separately the vitality of their photobionts and reproduction structures. As new species we have included the endolithic communities Chroococcidiopsis, Anabaena and Nostoc, and the vagant lichen species Aspicilia fruticulosa, after checking their suitability for this mission. Preparatory space simulation studies (UV Solar spectrum radiation and vacuum 10-2 $\mathrm{Pa}$ ) performed at the Spasolab-Laboratory of INTA (March-April 2007), have demonstrated a high survival rate of lichen species. Step 3 of Lithopanspermia will be tested with Rhizocarpon geographicum on its granitic rock substrate (taken as a model system), integrated and protected by an ablative material cover. It will be accommodated in the thermal protection shield of the Foton capsule, simulating in this way the external layer of a meteorite. After flight, analysis of the biological activity (chlorophyll a-fluorescence) will be performed on epilithic samples, Confocal Scanning Laser Microscopy will demonstrate vitality of all samples, and ultrastructural changes will be detected by Transmission Electron Microscopy and Cryoscanning. For the Lithopanspermia-Stone part additionally mineralogic- and petrologic studies will be performed to check compositional and structural changes.

de la Torre, R. et al. (2007). BIOPAN experiment LICHENS on the Foton-M2 mission: pre-flight verification tests of the Rhizocarpon geographicum-granite ecosystem. Adv. Space Res. in press, available on-line doi:10.1016/jasr.2007.02.022.

Sancho, L. et al. (2007). Lichens survive in space. Astrobiology 7, 443-454.

Stöffler, D. et al. (2007). Experimental evidence for the potential impact ejection of viable microorganisms from Mars and Mars-like planets. Icarus 186, 585-588.

Horneck, G. et al. (2007). Microbial rock inhabitants survive hypervelocity impacts on Mars-like host planets: First phase of Lithopanspermia experimentally tested. Astrobiology accepted for publication.
P8.2 High speed impact experiment for studying of survivability of micro organisms under low temperature

A. K. Pavlov 1,2, V. N. Shelegedin ${ }^{3}$, V. T. Kogan 1 , B. G. Zhukov', R. O. Kurakin ${ }^{1}$, S. I. Rozov ${ }^{1}$ * M. A. Vdovina ${ }^{1}$, A. V. Tretyakov ${ }^{1}$

${ }^{1}$ Ioffe Physico-Technical Institute, St. Petersburg, Russia

${ }^{2}$ Russian Astrobiology Center, St. Petersburg, Russia

${ }^{3}$ St. Petersburg Polytechnical State University, St. Petersburg, Russia e-mail:mariya.vdovina@mail.ioffe.ru

In our experiments we used the special small size accelerator - 'high speed' railgun, which was designed in the Ioffe Physico-Technical Institute. This railgun can launch small $(1-3 \mathrm{~mm})$ plastic impactors with velocities up to $7 \mathrm{~km} / \mathrm{s}$. Such velocities can be achieved at normal atmospheric pressure and correspond to dynamic pressure levels up to $40 \mathrm{GPa}$ on the surface of target. The target is kept at temperature of liquid nitrogen during the impact.

Two main fields of our research are survivability of microorganisms and synthesis of complex organic molecules under high-pressure shock waves. The ability of microorganisms to survive a short pulse of high pressure was studied in the first series of experiments. In these experiments we managed to achieve pressure up to $30 \mathrm{GPa}$. We used a frozen water solution containing cells of Vibrio sp. $\mathrm{X}$ in the middle phase of exponential growth $\left(\mathrm{CFU} \sim 108 \mathrm{ml}^{-3}\right.$ ) as a target. After the impact a decrease of survival up to 30 times in comparison with the control sample was discovered.

\section{P8.3 Estimation of possibilities of mass transfer} from Mars to Earth

M. Gorski, *S. Janikowski, M. Kubiak, G. Wiktorowicz Astronomical Observatory, Warsaw University, Poland

In our project we estimate the mass of matter that escapes from the reach of Mars's gravitational interaction as a result of a collision of objects such as asteroids and comets with the Mars's surface and afterwards obtains orbits that allow mass particles to hit the surface of Earth.

On the basis of spatial distribution of masses and velocities of matter that leaves Mars we find all possible closed orbits lying in the ecliptic plane and leaning to it that have common points with Earth's orbit in various phases of existence of the Solar System.

We analyse variations of quantity of matter that obtains orbits that have common points with Earth's orbit that result from variations of the stream of impactors. We also discuss possible modifications and improvements to our calculations such as gravitational interaction with Earth and other planets of the Solar System.

We also show examples of known mass transfers from Mars to Earth and results of experiments where impacts of planets with smaller objects were analysed. 
P8.4 Realization of Panspermia phase 2 inside the Mini External Exposure Facility on the International Space Station (ISS)

Cornelia Meyer ${ }^{1}$ *Ulrike Pogoda de La Vega ${ }^{2}$, Ralf Moeller ${ }^{2}$, Jean-Pierre de Vera ${ }^{3}$, Thomas Berger ${ }^{2}$, Guenther Reitz ${ }^{2}$, Petra Rettberg ${ }^{2}$, Uwe Reimold ${ }^{1}$, Rogier Schonenborg ${ }^{4}$

${ }^{1}$ Humboldt University Berlin, Museum of Natural History, Institute of Mineralogy, Invalidenstrasse 43, D-10115 Berlin, Germany

${ }^{2}$ German Aerospace Center (DLR), Institute of Aerospace Medicine, Radiation Biology division, Astrobiology working group, Linder Hoehe, D-51170 Cologne, Germany

${ }^{3}$ Heinrich-Heine-University of Duesseldorf, Institute of Botany 1, Universitaetsstr.1, D-40225 Duesseldorf, Germany

${ }^{4}$ Schonenborg Space Engineering B. V. (under contract in the Human Exploration Promotion Division), Directorate of Human Spaceflight, Microgravity and Exploration Programmes, ESA-ESTEC, Keplerlaan 1, POBox 299, 2200 AG Noordwijk ZH, The Netherlands

Arrhenius Panspermia theory has been slightly modified and is nowadays known as 'Lithopanspermia': its theorem is that viable microorganisms can be dispersed in space and transported between terrestrial planets by means of meteorites. In this scenario, the microorganisms have to cope with three major issues: (i) escape from the planet by impact ejection, (ii) journey through space over extended periods, and (iii) landing on another planet.

Recently the first phase of the transfer of life between Mars and Earth, i.e. the impact ejection, has been thoroughly investigated. We provided experimental data that Bacillus subtilis endospores as well as epilithic microbial communities such as the lichen Xanthoria elegans can endure shock pressures $>40 \mathrm{GPa}$, when embedded between rock layers (Meyer et al. 2007). Although step two of the scenario has been studied in space experiments and simulation facilities, these experiments have always been conducted under 'sterile and protective conditions'. The exposed bacteria and lichens were accommodated in specially designed anthropogenic hardware manufactured to endure the space environment. In contrast, rock material is subject to weathering during its travel through space: its shielding potential is reduced and eventually its chemical composition changes with increasing travel duration.

Based on these perceptions the recent experiments of phase 1, in the course of the research program SPP 1115 'Mars and terrestrial planets' project 'Meteorite impact and life' (funded by the DFG, German Research Foundation), have been conducted to simulate the natural case as closely as possible. The proposal to set up a close-to realistic experiment for the 'Lithopanspermia' 'travel through space' phase 2 emerged when this research group decided to participate to ESA's SUCCESS Student Contest with the perspective to place an experiment onto the ISS. A feasibility assessment for the realization of Panspermia on the ISS is currently ongoing.

We designed a cube of gabbro slabs to simulate the presence of biological material in different depths inside a meteorite and its composition. In order to simulate the free-floating motion of a meteorite it shall be attached to the ISS by an extension arm via the Mini External Exposure Facility. This experiment is not only designed to ascertain whether terrestrial biological systems are capable of surviving extended travel through space, but also to educe space weathering effects of lithic material such as gabbro and the effect of eventually resulting chemical changes on the micro-environment inhabited by the biological systems and to monitor space radiation and temperature variation with the help of TLD dosimeters.

Meyer et al. (2007). Shock experiments in support of the Lithopanspermia theory. 7th EANA workshop.
P8.5 Formation of oligopeptides in frozen solution: possibility of delivering seeds of life by small Solar System bodies

*N. Gontareva ${ }^{1}$, E. Kuzicheva ${ }^{1}$, A. Pavlov ${ }^{2}$, M. Vdovina ${ }^{2}$

${ }^{1}$ Laboratory of exobiology, Institute of cytology, St Petersburg, Russia

${ }^{2}$ Ioffe Physico-Technical Institute, St Petersburg, Russia

The dense cold phase of ISM hosts icy dust grains, which are an important chemical catalyst during its life cycle. Such particles consist of mineral core composed by silicate or olivine admixed with metal sulphides and oxides, with the water-icy envelope containing organic molecules.

The presence of organics in space (molecular hydrogen, water, $\mathrm{CO}$, $\mathrm{HCN}$, etc.) was proven by radioastronomic observations and laboratory investigations of meteorite matter and sample-return missions' materials. Organic molecules in the ISM evolve and become later incorporated in Solar System material (comets and meteorites). The heterogeneous porous structure of cometary nucleus and the abundance of water ice makes plausible further chemical processing of simple molecules, while availability of diverse impacts during space voyages in the Solar System provide the energy supply of a different spectrum range. Newly formed substances may possibly be shielded by cometary mineral structures and in subsurface cavities.

On the other hand, the presence of some organic matter in space is not enough for planetary evolution triggering. The problem of safe delivery to the surface may be solved due to the great amount of biomass supplement by micrometeorites (from 50 to $500 \mu \mathrm{m}$ in size) $-50-100$ tons daily.

Having polypeptides within the icy matrix, small bodies are transported to warm, dense, and active protostellar regions, where ultraviolet irradiation may become important and alter the grain composition. Furthermore UVC radiation may contribute to the formation of additional amounts of polypeptides, since short-wave photons are totally adsorbed by a thin outer layer. This presumption coincides with our previous investigations concerning UV impact on prebiotic formation of the main biological molecules. Combining two irradiation types in different stages of interstellar flight could compensate the effects of low reagents concentration and temperature. Since the primordial Earth had no atmosphere, the natural carriers could get freely onto its surface and thus raise the concentration of organic molecules.

The formation of polypeptides from single aminoacids was traced in simulation experiments representing the cometary structure. The experimental chamber was irradiated at subzero temperatures at the dosage of $2.54 \mathrm{kRad} / \mathrm{min}$. Solid frozen solutions of Gly and Phe were taken as the experimental samples inserted into the metal tube kept at subzero temperatures in the presence of liquid nitrogen. The formation of di- and tri-peptides was demonstrated after applying massspectrometry and HPLC techniques.

\section{P8.6 Plant seeds as biological instruments for dispersing life through the Universe in directed panspermia}

${ }^{*}$ David Tepfer ${ }^{1}$, Andreja Zalar ${ }^{1}$, Soren V. Hoffmann ${ }^{2}$, Sydney Leach ${ }^{3}$

${ }^{1}$ Institut National de la Recherche Agronomique, Versailles, France

${ }^{2}$ Institute for Storage Ring Facilities (ISA), University of Aarhus, Ny Munkegade, 8000 Aarhus $C$, Denmark

${ }^{3}$ LERMA, Observatoire de Paris-Meudon, 5 Place Jules Janssen, 92195 Meudon, France

Plant seeds are encapsulated, dormant, higher life forms, capable of withstanding harsh conditions over long periods of time. They evolved to protect the genome during dispersal. Dispersal, either active or passive, is a characteristic of essentially all organisms. The human propensity for self-dispersal is demonstrated by our space explorations. In contrast to humans, plant seeds are naturally resistant to conditions found in space, including ultra high vacuum, temperature extremes, UV light and cosmic radiation. For example, Arabidopsis seeds are 
insensitive to 105 times the dose of UV $(254 \mathrm{~nm})$ that completely kills the most resistant bacterial spores. Seeds can be further coated with protective and nutritive substances, and they can carry endophytic microorganisms within their tissues. Since some seeds are small, they could be carried into space in large numbers and accelerated toward distant targets.

\section{P9. Public outreach and education}

\section{P9.I SOWA - Polish National Astrobiology Student's Conference \\ K. Adamala ${ }^{1}$, M. Gochna ${ }^{2},{ }^{*}$ M. Gorski ${ }^{2}$, I. Kowalska ${ }^{2}$ \\ ${ }^{1}$ Faculty of Chemistry, University of Warsaw, Poland \\ ${ }^{2}$ Astronomical Observatory, University of Warsaw, Poland \\ e-mail:mareksgorski@yahoo.com}

The National Astrobiology Student's Conference was organized by the University of Warsaw Student's Scientific Association of Astronomers. The conference took place in May 2007 at the Faculty of Physics, University of Warsaw. It was the first such a conference in our country. Although Polish scientists are very active in this field of science, until now there was no meeting dedicated exclusively to astrobiology. Lecturers were the most prominent Polish scientists, representing all major subdisciplines of astrobiology. Lectures were given, inter alia, by astronomy professors Alex Wolszczan and Andrzej Udalski, astrophysics professors Ewa Szuszkiewicz and Michal Rózyczka, biogeology professor Jozef Kazmierczak and cosmochemistry professor Zbigniew Kisiel. Guests could hear lectures about origin, evolution and the search for extrasolar planets, exploration of the Solar System and about searching for prebiotic compounds in interstellar space. Lectures about extremophiles, microorganisms and problems of human presence in outer space were also given. Participants were undergraduate and graduate students, as well as $\mathrm{PhDs}$, professors and non-academic people from all major Polish cities. Guests from the University of Warsaw, University of Szczecin, Warsaw University of Technology, Nicolaus Copernicus University of Torun and Adam Mickiewicz University of Poznan were present. The workshop was highly appreciated, and it was decided that it will be repeated. Next year the conference will be organized by our colleagues from the University of Szczecin. We hope to witness the beginning of the new tradition. To the best of our knowledge, it will be the first national, annual astrobiology workshop organized by undergraduate students in Europe.

\section{P9.2 Teaching \& the public outreach in the field of astrobiology: Finland as an example \\ Marianna Ridderstad \\ Observatory, Helsinki University, Helsinki, Finland}

The brief history of teaching astrobiology in Finland is given. The current situation of teaching astrobiology and the public outreach in the field of astrobiological research is examined globally, in Europe and, specifically, in Finland. The educational roles of planetariums, science centres, television, books, magazines and the Internet are each discussed and, finally, compared. Future prospects in educational astrobiology in Finland and elsewhere are discussed.

\section{P9.3 Perspectives on Astrobiology projects in Brazil} *E. Janot-Pacheco ${ }^{1}$ C. Lage $^{2}$, H. Boechat-Roberty ${ }^{3}$

G. Porto-De-Mello ${ }^{3}$, A. Wuensche $e^{4}$, A. Friaça ${ }^{1}$

${ }^{1}$ Dept Astronomia, Instituto de Astronomia, Geofisica e Ciencias

Atmosfericas, Universidade de Sao Paulo, SP, Brazil

${ }^{2}$ Prog Biol Mol \& Estrutural, Instituto de Biofisica Carlos Chagas Filho, Universidade Federal do Rio de Janeiro, RJ, Brazil

${ }^{3}$ Observatorio do Valongo, Universidade Federal do Rio de Janeiro, RJ, Brazil

${ }^{4}$ Divisao de Astrofisica, Instituto Nacional de Pesquisas Espaciais, Sao Jose dos Campos, Brazil

A nascent Brazilian group has started projects in the field of Astrobiology in the past year. The initiative has been powered after the organization of the I Brazilian Workshop on Astrobiology (BWA) in March 2006, the first meeting on the subject in Brazil. The increasing number of papers in this area in the last few years is noticeable, coming mainly from Astronomy, Biology, Chemistry and Geology fields. Presently in Brazil there are astronomers searching for exoplanets, trying to understand the formation of planetary discs and, along with chemists, studying the chemistry of strategic molecules interstellar medium, particularly searching for organic compounds. Biologists have focused on extremophiles, studying their survival conditions and evolution in environments under extreme conditions (high temperature, radiation, pressure, salinity and $\mathrm{pH}$ ). Geologists have worked on the investigation of atmospheric and geologic records of the young Earth.

\section{PIO. Beyond the Solar System}

\section{PI0.I Laboratory set-up for studying ices at the}

\section{5-1000 microns region}

*J. Canto, O. Gomis, R. Vilaplana, M. Domingo

Escola Politècnica Superior d'Alcoi, Universitat Politècnica de València, Spain

Simple molecules, such as $\mathrm{H}_{2} \mathrm{O}, \mathrm{CO}$, and $\mathrm{CO}_{2}$ in form of ices, are present condensed on carbonaceous and silicatic grain mantles in dense interstellar clouds and circumstellar environments. The presence of those ices is inferred from the analysis of spectra mainly in the mid infrared region. In the near future Herschel and ALMA missions will provide new spectral data in the almost unexplored far infrared and sub-millimetre part of the spectrum. In our laboratory the silicon bolometer of our Bruker IFS 66s/V let us obtain high quality in the FIR region $(25-1000 \mu \mathrm{m})$ under vacuum conditions. This detector gives us a sensitivity four orders of magnitude greater than that obtained with DTGS detectors working at room temperature. The FIR spectra we have planned to obtain will serve to characterize in the far infrared some simple ices and their mixtures and also to compare observational data with laboratory data. It is also planned to do a systematic laboratory study of the effects that UV photolysis and thermal annealing have in the ice band profiles and in the ice structure.

\section{PI0.2 Molecular line carriers in the translucent clouds}

*M. Kazmierczak ${ }^{1}, J$. Krelowski ${ }^{1}, M$. Schmidt ${ }^{2}$

${ }^{1}$ Nicolaus Copernicus University, Centre for Astronomy, Torun, Poland ${ }^{2}$ Nicolaus Copernicus Astronomical Center, Torun, Poland

We present high-resolution and high signal-to-noise spectroscopic observations of interstellar molecular lines of $\mathrm{CN}, \mathrm{CH}, \mathrm{CH}+$ and $\mathrm{C}_{2}$ towards early-type stars. Objects with intermediate colour excess and single velocity component were selected to be sure that molecular lines originate from single clouds. The curve of growth method is applied to equivalent widths for determination of column densities. The excitation temperature is extracted from the rotational diagrams for $\mathrm{C}_{2}$. Comparison with the theoretical model of excitation of $\mathrm{C}_{2}$ allows inference of densities of translucent clouds. 


\section{PI0.3 New redox-catalysts found in interstellar} dust by $\mathrm{MS}$

Franz R. Krueger

Engineering office Dr. Krueger, Germany

New redox-catalysts, related to the PQQ-enzymes were found in interstellar dust. The active group has three oxidation states, a quinone, a hydroquinone, and an intermediate radical. Up to now it was believed that synthesis of these important and ubiquitous molecules in living systems needed already RNA. So the PQQ-enigma may be solved by accepting a stellar dust trigger.

\section{PI0.4 Investigations of dusty circumstellar disks \\ *R. Nilsson, G. Olofsson \\ Stockholm Observatory, Stockholm University, AlbaNova University Center, Stockholm, Sweden}

The evolution of circumstellar disks, from initial swirling dust and gas in the proto-stellar collapse, into full-fledged planetary systems, is of strong astrobiological significance, and many questions remain to be answered. Replenishment of dust by collisions and release from comets keeps the disk dusty, long after the gas has photoevaporated or been incorporated into planets. At Stockholm Observatory, research investigating the physical structures of circumstellar disks, primarily debris disks, is carried out by using imaging observations. This poster briefly presents two main projects and initial results: observations of scattered optical light with the polarizing coronagraph instrument PolCor, developed by Göran Olofsson, and used on NOT; and sub-mm observations with the LABOCA instrument on APEX. In the first project, properties of circumstellar dust disks are examined with an instrument using a high-speed CCD in conjunction with a coronagraphic technique, where the direct light from the star is blocked out, making the faint scattered optical light from the disk detectable. By also inserting a polarizing filter in different orientations, the angle and degree of polarization can be calculated and used to further enhance the contrast. This high-contrast imaging of low surface-brightness features can reveal gaps, knots, and asymmetries in the disks, perhaps indicating planets. In the second project, LABOCA has been used to study a number of main-sequence stars with known ages and inferred disks (from mid- to far-infrared excess) at 870 microns, to probe extended, cold dust components (out to some hundreds of AU), in order to assess the existence, extent and mass of Kuiper-Belt-like disk structures. Together with the fairly accurately known ages of the stars, evolutionary aspects of the disks can be studied.

\section{PI0.5 Radiative transfer in protoplanetary disk: effects on physical and chemical properties of gas and presolar dust}

L. Graziani ${ }^{1},{ }^{*}$ S. Aiello ${ }^{1}$, A. Belleni ${ }^{2}$, G. Briani ${ }^{1}$, C. Cecchi-Pestellini ${ }^{3}$

${ }^{1}$ Department of Astronomy and Space Science, University of Florence, Largo Fermi 2, 50125 Firenze, Italy

e-mail: aiello@arcetri.astro.it

${ }^{2}$ Department of Civil Engineering, University of Florence, Via Santa

Marta 3, 50139 Firenze, Italy

${ }^{3}$ Osservatorio Astronomico di Cagliari, Loc. Poggio dei Pini, Strada 54, 09012 Capoterra - CA, Italy

Protoplanetary disks are the precursors of planetary systems. All building materials needed to assembly the planetary systems are supplied by these reservoirs, including many organic molecules (Ehrenfreund \& Charnley 2000; Markwick \& Charnley 2004). Thus, the physical and chemical properties in protoplanetary disks set the boundary conditions for the formation and evolution of planets and other Solar System bodies.

The structure and chemistry of protoplanetary disks depend strongly on the nature of the central star around which they have formed. The dust temperature is manly set by the stellar luminosity, while the chemistry of the whole disk depends on the UV and X-ray fluxes. Therefore, a knowledge as accurate as possible of the radiative transfer inside disks is a prerequisite for their modelling.
In a passive accretion disk the mass accretion processes, the viscous dissipative heating, and the reprocessing of stellar radiation by the flared disk atmospheres, may play a primary role in structuring the various radial regions at different evolutionary epochs making difficult to provide a 'standard model' for this scenario. On the other hand, our current knowledge of the star-forming regions and the more evolved debris disks let us to attempt a disk-star environment modelling.

During the last 10 years many authors suggested various numerical and analytical techniques to resolve the disk temperature structure and provide vertical temperature profiles and disk SED reconstruction (Chiang \& Goldreich 1997; D’Alessio et al. 1998; Bjorkman \& Wood 2001; Dullemond \& Natta 2003). In this work we have solved the radiative transfer problem in separate interesting disk regions: (1) disk atmospheres at large radius, $r>10 \mathrm{AU}$; (2) equatorial plane regions spanned over $1<r<10 \mathrm{AU}$ and $10<r<100 \mathrm{AU}$. The effects of different dust compositions (Draine \& Lee 1984; Henning \& Stognienko 1996) and ranges of star luminosity in UV and X-rays have been compared. We solved the equatorial plane problem in cylindrical symmetry modelling the disk as an internal high-density cloud region heated primarily by viscous processes and X-UV star radiation from upper atmosphere. In that region we applied the P-N approximation (Pomraning 2000) obtaining a rapid convergent solution and customizable accuracy at boundaries. As a complementary task we investigate more complex atmosphere models where the diffuse gas-dust medium, the direct high-energy X-UV star flux and the geometry of the disk impose a more accurate treatment. We propose a simplified analytical approach based on P-N approximation as a parallel plane problem (suitable for a limited range of $r$ ) and a more accurate Monte Carlo integration with specifically designed numerical code solving the $\mathrm{RT}$ in the four Stokes radiation field components. The resulting radiation fluxes of non-reprocessed UV and X-rays provide initial boundary conditions for the inner equatorial region. Some preliminary results are presented.

Bjorkman, J. E. \& Wood, K. (2001). Astrophys. J. 554, 615.

Dullemond, C. P. \& Natta, A. (2003). Astron. Astrophys. 405, 597-605. Chiang, E. I. \& Goldreich, P. (1997). Astrophys. J. 490, 368.

Dalessio, P., Canto, J., Calvet, N. \& Lizano, S. (1998). Astrophys. J. 500, 411.

Draine, B. T. \& Lee, H. M. (1984). Astrophys. J. 285, 89

Ehrenfreund, P. \& Charnley, S. B. (2000). Ann. Rev. Astron. Astrophys. 38, 427-483.

Henning, T. H. \& Stognienko, R. (1996). Astron. Astrophys. 311, 291-303.

Markwick, A. J. \& Charnley, S. B. (2004). Astrobiology: Future Perspectives, ed. Eherenfreund, P. et al., pp. 33-66. Kluwer, Dordrecht. Pomraning, G. (2000) The Equations of Radiation Hydrodynamics, p. 71. Dover.

\section{PI0.6 Transiting extrasolar planets: the photometric information \\ Alvaro Gimenez}

European Space Agency, Paris, France and Centro de Astrobiologia, Madrid, Spain

The number of extrasolar planets undergoing transits in front of their parent stars is steadily increasing and will certainly rise dramatically with the results obtained from space missions like Corot or Kepler.

The obtention of analytical equations for the photometric behaviour of the transits, as well as the non-orbital component of the radial velocity curve, is shown. Further use of these expressions is presented leading to accurate estimation of the star-planet system parameters with very limited photometric information.

Finally, the current situation with respect to the physical characteristics of all planets showing transits is reviewed. 


\section{PI0.7 Influence of stellar X-ray luminosity evolution on exoplanetary mass distributions}

*T. Penz ${ }^{1}$, G. Micela ${ }^{1}$, H. Lammer ${ }^{2}$

${ }^{1}$ INAF-Osservatorio Astronomico di Palermo, Palermo, Italy

${ }^{2}$ Space Research Institute, Austrian Academy of Sciences, Graz, Austria High-energy stellar luminosity varies over a wide range for $\mathrm{G}$ field stars We investigate the temporal evolution of the distribution of stellar Xray luminosity and its influence on the evolution of close-in exoplanets. X-ray luminosity distributions from the Pleiades, the Hyades and the field are used to derive a scaling law for the evolution of the stellar Xray luminosity distribution. A modified energy-limited escape approach is used to calculate planetary mass loss for a broad range of planetary parameters. We show that the evolution of close-in exoplanets strongly depends on the detailed X-ray luminosity history of its host star, which varies over several orders-of-magnitude for $\mathrm{G}$ stars. Stars located at the high-energy tail of the luminosity distribution can evaporate most of their planets within $0.5 \mathrm{AU}$, while for a moderate luminosity a significant fraction of planets can survive. Additionally, we calculate the deviations from an initial planetary mass distribution caused by atmospheric escape.

\section{PI0.8 Counter-rotating planetary systems \\ * S. Kotiranta, H. Lehto, S. Mikkola}

Tuorla observatory and Department of Physics, University of Turku,

Finland

Counter-rotation is a phenomenon where some of the minor bodies moving around a more massive central body move against the direction of other ones or against the rotation of the central body. This type of motion is known to exist among the moons of the giant planets in our Solar System as well among some galactic nuclei. Currently no specific search to determine this aspect of dynamics in exoplanetary systems has been done, mostly because observing it is extremely difficult and actually quite clearly beyond the contemporary capacity of the observational instrumentation. However, this situation will change in the future.

In our study we discuss one possible existence of counter-rotation in the scale of a planetary system, most probably caused by a stellar flyby. We also demonstrate its highly stabilizing effect on the orbital motion and finally suggest that the presence of this phenomenon could be observationally detected in three different ways: by finding a planet within the normally unstable region, by seeking starspot transits and analyzing their frequency compared to the rotation period of the star or by analyzing the spectral shifts caused by the Rossiter-McLaughlin effect appearing when the planet obscures the central star.
PI0.9 The role of the inclination of terrestrial planets on the habitable zone's stability

${ }^{*}$ B. Funk ${ }^{1}$, R. Schwarz ${ }^{2}$, E. Pilat-Lohinger ${ }^{1}$, A. Süli ${ }^{2}$, R. Dvorak ${ }^{1}$

${ }^{1}$ Institute for Astronomy, University of Vienna, Vienna, Austria

${ }^{2}$ Department of Astronomy, Eötvös University, Budapest, Hungary

To investigate the long-term stability of a terrestrial planet in the habitable zone (HZ), we have to distinguish 3 different types of $\mathrm{HZs}$ :

Solar System Type: if the HZ is between the star and the giant planet (like in the Solar System)

Hot Jupiter Type: if the HZ is outside the giant planet (for systems with 'hot-Jupiters')

Trojan Type: if the giant planet moves in the HZ, then we can expect habitable Moons and/or habitable Trojan planets.

Since earlier calculations indicated that the stability of the HZ depends on the inclination of the terrestrial planets, we present a detailed numerical investigation with the main goal to show correlations between the eccentricity, the mass and the distance of the known planet for various inclinations of the terrestrial planets and the stability of the HZ.

To be able to apply our study to most of the discovered extrasolar planetary systems we defined, that the distance between the star and the gas giant is fixed to $1 \mathrm{AU}$ and studied all three $\mathrm{HZ}$-configurations as described above. As a model we used the elliptic restricted three body problem, which consists of a star (1 Msun) a planet (0.5 .. 5 Mjup) and mass-less test planets.

We found a quite interesting dependence between the stability of the $\mathrm{HZ}$ and the inclination of the test-planets.

\section{PI0.10 Giant planets as a dynamical shield - numerical studies \\ *T. Laakso, J. Rantala, M. Kaasalainen University of Helsinki, Finland}

The small-body impact rate may become a critical factor when evaluating the habitability of an extrasolar terrestrial planet. In a planetary system, giants, the gravitationally strongest players, have the most power to scatter the orbits, and sculpt the flux of small-bodies. Hence, the giants can act as a dynamical shield for terrestrial planets inside the habitable zone (HZ). In our Solar System, Jupiter has traditionally been thought as an invaluable protector for the Earth.

In order to compare giant-planet systems with respect to their efficiency to act as a dynamical shield, we present a numerical tool where we integrate the giant planets numerically along with a large number of hypothetical small bodies that are initially in eccentric HZ-crossing orbits. Our analysis produces a single number, the escape rate, which represents the rate at which the small-body flux is perturbed away by the giant planets into orbits that no longer pose a threat to terrestrial planets inside the HZ. Obtaining the escape rate this way is similar to computing the largest Liapunov exponent as the exponential rate of divergence of two nearby orbits.

We present some computed escape rates on selected planetary systems, focusing on effects of varying the masses and semi-major axes of the giant planets. In the case of our Solar System we find rather surprisingly that Jupiter, in its current orbit, may provide a minimal amount of protection to the Earth. 